\title{
Anti-Inflammatory Activities of Natural Products Isolated from Soft Corals of Taiwan between 2008 and 2012
}

\section{Wen-Chi Wei ${ }^{1}$, Ping-Jyun Sung ${ }^{2,3}$, Chang-Yih Duh ${ }^{4}$, Bo-Wei Chen ${ }^{4}$, Jyh-Horng Sheu ${ }^{4,5,6, *}$ and Ning-Sun Yang $1,7,8, *$}

1 Agricultural Biotechnology Research Center, Academia Sinica, Taipei 128, Taiwan;

E-Mail: jackwei@gate.sinica.edu.tw

2 National Museum of Marine Biology \& Aquarium, Pingtung 944, Taiwan;

E-Mail: pjsung@nmmba.gov.tw

3 Graduate Institute of Marine Biotechnology, National Dong Hwa University, Pingtung 944, Taiwan

4 Department of Marine Biotechnology and Resources, National Sun Yat-sen University, Kaohsiung 804, Taiwan; E-Mails: yihduh@mail.nsysu.edu.tw (C.-Y.D.); a6152761@yahoo.com.tw (B.-W.C.)

5 Department of Medical Research, China Medical University Hospital, China Medical University, Taichung 404, Taiwan

6 Graduate Institute of Natural Products, Kaohsiung Medical University, Kaohsiung 807, Taiwan

7 Institute of Biotechnology, National Taiwan University, Taipei 106, Taiwan

8 Department of Life Science, National Central University, Taoyuan 320, Taiwan

* Authors to whom correspondence should be addressed; E-Mails: sheu@mail.nsysu.edu.tw (J.-H.S.); nsyang@gate.sinica.edu.tw (N.-S.Y.); Tel./Fax: +886-7525-2000 (ext. 5030) (J.-H.S.), +886-2-2787-2067 (N.-S.Y.).

Received: 30 July 2013; in revised form: 12 September 2013 / Accepted: 13 September 2013 / Published: 23 October 2013

\begin{abstract}
This review reports details on the natural products isolated from Taiwan soft corals during the period 2008-2012 focusing on their in vitro and/or in vivo anti-inflammatory activities. Chemical structures, names, and literature references are also reported. This review provides useful and specific information on potent anti-inflammatory marine metabolites for future development of immune-modulatory therapeutics.
\end{abstract}

Keywords: soft coral; anti-inflammatory activity; iNOS; COX-2; superoxide anion; elastase 


\section{Introduction}

Marine natural products, especially those from stationary or slow moving marine organisms, are used naturally as a chemical defense to protect the organisms from dangerous predators, stressful local environments, and/or the encroachment of competitors. Due to the biological and chemical diversity of marine habitats, and the identification and greater understanding of marine secondary metabolites with unique chemical structures and biological activities, natural products from marine organisms are increasingly being considered as a major source of new therapeutics [1-3]. More than 20,000 novel compounds have been isolated and identified from marine organisms since the 1960s [4]. At least two current drugs and a series of anti-tumor drug candidates in preclinical or clinical trials have been developed from marine natural products [2-4]. The soft corals or Alcyonacea, an order of Anthozoa widely distributed in warm seawaters, have been a particular focus of attention. An abundance of unique secondary metabolites including sesquiterpenoids, diterpenoids, steroids and other chemical compounds have been isolated and identified from various species of soft corals [5-7]. It has been estimated that the percentage of new metabolites discovered from soft corals represents up to $22 \%$ of the total new marine natural products reported from 2010 to 2011 [5,6]. Importantly, many of the natural products discovered from soft corals have been demonstrated to exhibit a spectrum of biological activities such as anti-tumor, antiviral, antifouling and anti-inflammatory [5-8].

Inflammation processes often constitute an initial activation of the mammalian immune system, and the body's normal defense or protective mechanisms in response to microbial infection or irritation or injury of tissues/organs. Increasing evidence suggests a critical link between inflammation and the chronic promotion/progression of various human diseases, including atherosclerosis, diabetes, arthritis, inflammatory bowel disease, cancer and Alzheimer. Proinflammatory enzymes, particularly the inducible nitric oxide synthase (iNOS) for nitric oxide production and cyclooxygenase (COX-2) for prostaglandin production, have been demonstrated to play central roles in the development of inflammatory diseases. In addition, it is also known that during the initial phase of acute inflammation, neutrophils are one of the first leukocyte populations to migrate towards the damaged tissue sites [9]. Neutrophils play a key role in the pathogenesis of various chronic inflammation diseases such as rheumatoid arthritis $[10,11]$. Activated neutrophils can secrete the superoxide anion, reactive oxygen species (ROS) and enzymes that are associated with the killing of invading pathogens [12]. Furthermore, elastase secreted by stimulated neutrophils has been recognized to play a key contribution in the demolition of tissues affected by chronic inflammatory disease [13]. Therefore, evaluation of the inhibition of iNOS and COX-2 expression, the production of superoxide anion, and the release of elastase in inflammatory cells/tissues by various natural products have been extensively employed in a spectrum of in vitro preliminary screening systems for lead compound or drug discovery. Recently, a number of marine biology and chemistry researchers in Taiwan (including our laboratory) have systematically screened several marine natural products isolated from soft corals for such in vitro anti-inflammatory activities, mainly by measuring the inhibition of iNOS, COX-2, superoxide anion or elastase in murine immune cells. Animal models were further used to evaluate the potential therapeutic activities of candidate compounds in specific disease models. This report reviews some recent representative studies and examples of marine natural products with anti-inflammatory and other related bioactivities that have been isolated from soft corals of Taiwan. Soft corals are 
abundant in the off-shore environment of the island of Taiwan, and have hence become a focus of local studies of marine nature products. We hope that this review will provide a useful data for the further study of marine natural products.

\section{Results and Discussion}

In the reports reviewed here, anti-inflammatory activities of natural products from the soft corals of Taiwan were generally determined in vitro by their inhibition of LPS-induced expression of iNOS and COX-2 in murine macrophage cells (RAW264.7) or by their inhibition of the production of superoxide anion and the release on the elastase from human neutrophils in response to FMLP/CB.

\subsection{Sesquiterpenoids}

\subsubsection{Triquinane-Type Sesquiterpenoids}

Table 1 summarizes nine triquinane-type sesquiterpenoids (1-9) evaluated for in vitro anti-inflammatory activity in literature published from 2008 to 2012. The corresponding chemical structures are reported in Figure 1.

Table 1. Chemical constituents of triquinane-type sesquiterpenoids from soft corals of Taiwan.

\begin{tabular}{|c|c|c|c|c|}
\hline No. & Name & Sources & Activities * & Reference \\
\hline 1 & $\Delta^{9(12)}$-Capnellene- $8 \beta, 10 \alpha$-diol & Capnella imbricata & $\mathrm{I}, \mathrm{C}$ & [14] \\
\hline 2 & $8 \alpha$-Acetoxy- $\Delta^{9(12)}$-capnellene- $10 \alpha$-ol & Capnella imbricata & $\mathrm{I}, \mathrm{C}$ & [14] \\
\hline 3 & $\Delta^{9(12)}$-Capnellene-10 $\alpha$-ol-8-one & Capnella imbricata & I & [14] \\
\hline 4 & $\Delta^{9(12)}$-Capnellene-8 $\beta, 15$-diol & Capnella imbricata & & [14] \\
\hline 5 & $\Delta^{9(12)}$-Capnellene- $8 \beta, 10 \alpha, 13$-triol & Capnella imbricata & & [14] \\
\hline 6 & $8 \beta, 10 \alpha$-Diacetoxy- $\Delta^{9(12)}$-capnellene & Capnella imbricata & & [14] \\
\hline 7 & $8 \beta$-Acetoxy- $\Delta^{9(12)}$-capnellene & Capnella imbricata & & [14] \\
\hline 8 & $\Delta^{9(12)}$-Capnellene- $8 \beta$-ol & Capnella imbricata & & [14] \\
\hline 9 & $\Delta^{9(12)}$-Capnellene-12-ol-8-one & Capnella imbricata & $\mathrm{I}, \mathrm{C}$ & [14] \\
\hline
\end{tabular}

* Inhibition of iNOS (I) and COX-2 (C).

Figure 1. The structures of triquinane-type sesquiterpenoids (1-9).

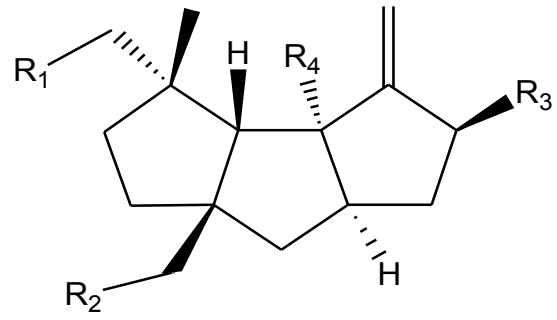

1: $\mathrm{R}_{1}=\mathrm{H}, \mathrm{R}_{2}=\mathrm{H}, \mathrm{R}_{3}=\mathrm{OH}, \mathrm{R}_{4}=\mathrm{OH} \quad$ 5: $\mathrm{R}_{1}=\mathrm{H}, \mathrm{R}_{2}=\mathrm{OH}, \mathrm{R}_{3}=\mathrm{OH}, \mathrm{R}_{4}=\mathrm{OH}$

2: $\mathrm{R}_{1}=\mathrm{H}, \mathrm{R}_{2}=\mathrm{H}, \mathrm{R}_{3}=\mathrm{OAc}, \mathrm{R}_{4}=\mathrm{OH}$ 6: $\mathrm{R}_{1}=\mathrm{H}, \mathrm{R}_{2}=\mathrm{H}, \mathrm{R}_{3}=\mathrm{OAc}, \mathrm{R}_{4}=\mathrm{OAc}$

3: $\mathrm{R}_{1}=\mathrm{H}, \mathrm{R}_{2}=\mathrm{H}, \mathrm{R}_{3}=\mathrm{O}, \mathrm{R}_{4}=\mathrm{OH} \quad$ 7: $\mathrm{R}_{1}=\mathrm{H}, \mathrm{R}_{2}=\mathrm{H}, \mathrm{R}_{3}=\mathrm{OAc}, \mathrm{R}_{4}=\mathrm{H}$

4: $\mathrm{R}_{1}=\mathrm{OH}, \mathrm{R}_{2}=\mathrm{H}, \mathrm{R}_{3}=\mathrm{OH}, \mathrm{R}_{4}=\mathrm{H} \quad$ 8: $\mathrm{R}_{1}=\mathrm{H}, \mathrm{R}_{2}=\mathrm{H}, \mathrm{R}_{3}=\mathrm{OH}, \mathrm{R}_{4}=\mathrm{H}$

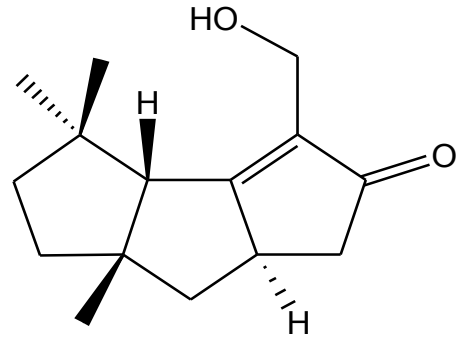

9 


\subsubsection{Nardosinane-Type Sesquiterpenoids}

Table 2 summarizes seven nardosinane-type sesquiterpenoids (10-16) evaluated for in vitro anti-inflammatory activity in literature published from 2008 to 2012. The corresponding chemical structures are reported in Figure 2.

Table 2. Chemical constituents of nardosinane-type sesquiterpenoids from soft corals of Taiwan.

\begin{tabular}{ccccc}
\hline No. & Name & Sources & Activities * & Reference \\
\hline $\mathbf{1 0}$ & Paralemnolin J & Paralemnalia thyrsoides & {$[15]$} \\
$\mathbf{1 1}$ & Paralemnolin K & Paralemnalia thyrsoides & & {$[15]$} \\
$\mathbf{1 2}$ & Paralemnolin L & Paralemnalia thyrsoides & & {$[15]$} \\
$\mathbf{1 3}$ & Flavalin A & Lemnalia flava & I,C & {$[16]$} \\
$\mathbf{1 4}$ & Flavalin B & Lemnalia flava & & {$[16]$} \\
$\mathbf{1 5}$ & Flavalin C & Lemnalia flava & {$[16]$} \\
$\mathbf{1 6}$ & Flavalin D & Lemnalia flava & {$[16]$} \\
\hline \multicolumn{5}{c}{ * Inhibition of iNOS (I) and COX-2 (C). }
\end{tabular}

Figure 2. The structures of nardosinane-type sesquiterpenoids (10-16).

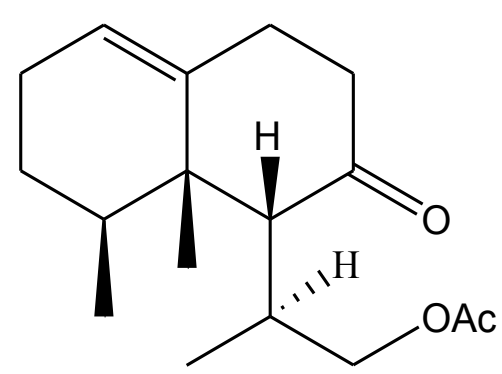

10

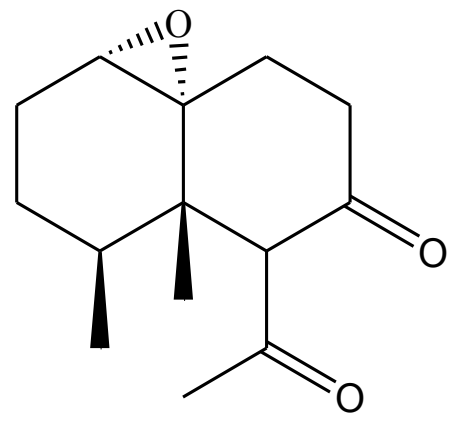

11<smiles>CC(=O)OCC(C)(O)C1=C[C@@]2(C)C(=CCC[C@@H]2C)CC1</smiles>

12

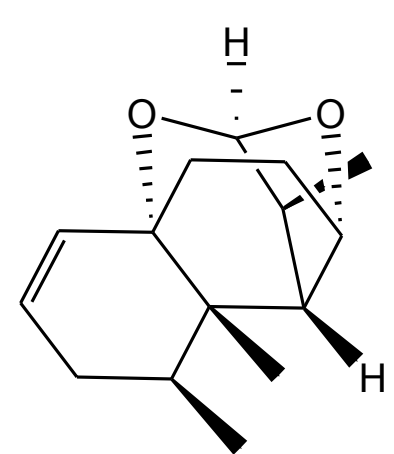

13

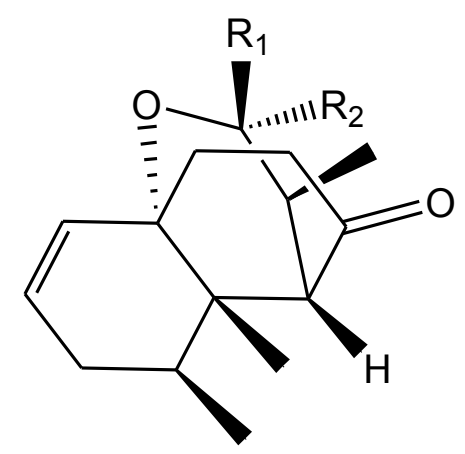

14: $\mathrm{R}_{1}=\mathrm{H}, \mathrm{R}_{2}=\mathrm{OH}$

15: $\mathrm{R}_{1}=\mathrm{H}, \mathrm{R}_{2}=\mathrm{OMe}$

16: $\mathrm{R}_{1}=\mathrm{OMe}, \mathrm{R}_{2}=\mathrm{H}$ 


\subsubsection{Aromadendrane-Type Sesquiterpenoids}

Table 3 summarizes six aromadendrane-type sesquiterpenoids (17-22) evaluated for in vitro anti-inflammatory activity in literature published from 2008 to 2012. The corresponding chemical structures are reported in Figure 3.

Table 3. Chemical constituents of aromadendrane-type sesquiterpenoids from soft corals of Taiwan.

\begin{tabular}{ccccc}
\hline No. & Name & Sources & Activities * & Reference \\
\hline $\mathbf{1 7}$ & Lochmolin A & Sinularia lochmodes & C & {$[17]$} \\
$\mathbf{1 8}$ & Lochmolin B & Sinularia lochmodes & C & {$[17]$} \\
$\mathbf{1 9}$ & Lochmolin C & Sinularia lochmodes & & {$[17]$} \\
$\mathbf{2 0}$ & Lochmolin D & Sinularia lochmodes & & {$[17]$} \\
$\mathbf{2 1}$ & Lochmolin E & Sinularia lochmodes & C & {$[17]$} \\
$\mathbf{2 2}$ & Lochmolin F & Sinularia lochmodes & C & {$[17]$} \\
\hline \multicolumn{5}{c}{ * Inhibition of COX-2 (C). }
\end{tabular}

Figure 3. The structures of aromadendrane-type sesquiterpenoids (17-22).

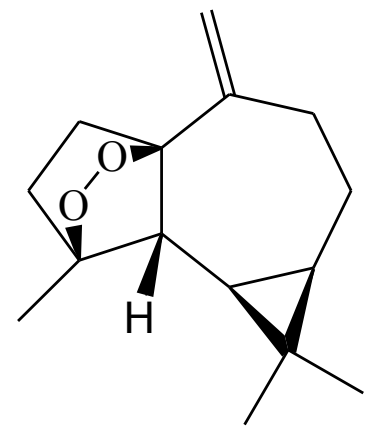

17

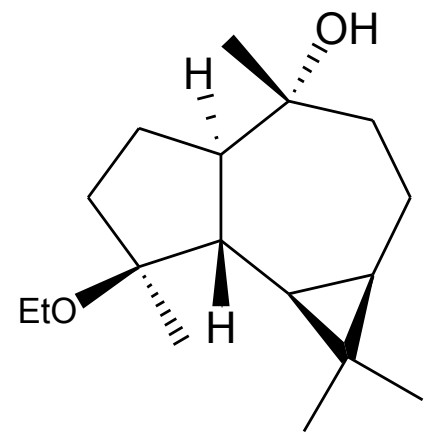

18

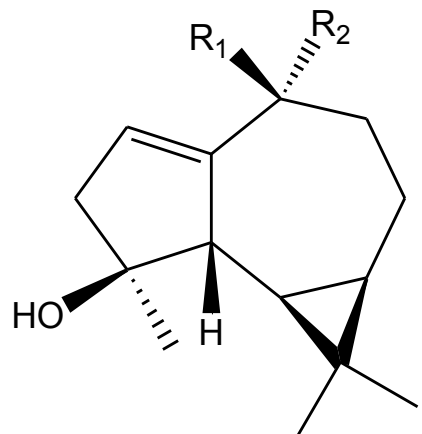

19: $\mathrm{R}_{1}=\mathrm{OOH}, \mathrm{R}_{2}=\mathrm{CH}_{3}$ 20: $\mathrm{R}_{1}=\mathrm{CH}_{3}, \mathrm{R}_{2}=\mathrm{OH}$

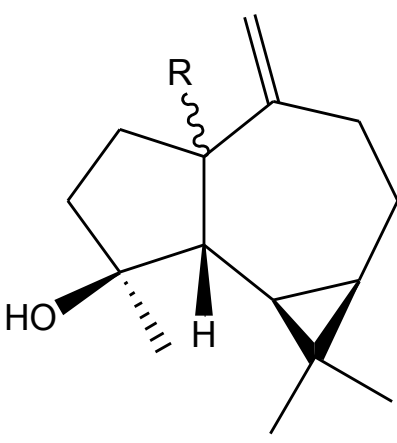

21: $\mathrm{R}=\beta-\mathrm{OOH}$ 22: $\mathrm{R}=\alpha-\mathrm{OOH}$

\subsubsection{Selinane- and Oppositane-Type Sesquiterpenoids}

Table 4 summarizes four selinane- and oppositane-type sesquiterpenoids (23-26) evaluated for in vitro anti-inflammatory activity in literature published from 2008 to 2012. The corresponding chemical structures are reported in Figure 4.

Table 4. Chemical constituents of selinane- and oppositane-type sesquiterpenoids from soft corals of Taiwan.

\begin{tabular}{cclcc}
\hline No. & Name & \multicolumn{1}{c}{ Sources } & Activities * & Reference \\
\hline $\mathbf{2 3}$ & $1 \beta$-Hydroxy-6 $\alpha$-acetoxyeudesm-4(15)-ene & Sinularia leptoclados & & {$[18]$} \\
$\mathbf{2 4}$ & $1 \beta, 6 \alpha$-Dihydroxyeudesm-4(15)-ene & Sinularia leptoclados & I & {$[18]$} \\
$\mathbf{2 5}$ & Leptocladolin A & Sinularia leptoclados & & {$[18]$} \\
$\mathbf{2 6}$ & Leptocladolin B & Sinularia leptoclados & & {$[18]$} \\
\hline
\end{tabular}

* Inhibition of iNOS (I). 
Figure 4. The structures of selinane- and oppositane-type sesquiterpenoids (23-26).<smiles>[R][C@@H]1[C@@H](C(C)C)CC[C@]2(C)[C@@H](O)CCC(=C)[C@@H]12</smiles>

23: $\mathrm{R}=\mathrm{Ac}$<smiles>C=C1CC[C@@H](O)[C@]2(C)CC[C@H](C(OC(C)=O)C(C)C)[C@H]12</smiles>

25<smiles>C=C1CC[C@@H](O)[C@@]2(C)CC[C@H](CC(C)(C)OC(C)=O)[C@H]12</smiles>

26

\subsubsection{Ylangene-Type Sesquiterpenoids}

Table 5 summarizes three ylangene-type sesquiterpenoids (27-29) evaluated for in vitro anti-inflammatory activity in literature published from 2008 to 2012. The corresponding chemical structures are reported in Figure 5.

Table 5. Chemical constituents of ylangene-type sesquiterpenoids from soft corals of Taiwan.

\begin{tabular}{ccccc}
\hline No. & Name & Sources & Activities * & Reference \\
\hline $\mathbf{2 7}$ & $(1 S, 2 S, 4 R, 6 S, 7 R, 8 S)-4 \alpha$-Formyloxy- $\beta$-ylangene & Lemnalia flava & I,C & {$[16]$} \\
$\mathbf{2 8}$ & Lemnalol & Lemnalia flava & & {$[16]$} \\
$\mathbf{2 9}$ & Isolemnalol & Lemnalia flava & & {$[16]$} \\
\hline
\end{tabular}

* Inhibition of NOS (I) and COX-2 (C).

Figure 5. The structures of ylangene-type sesquiterpenoids (27-29).

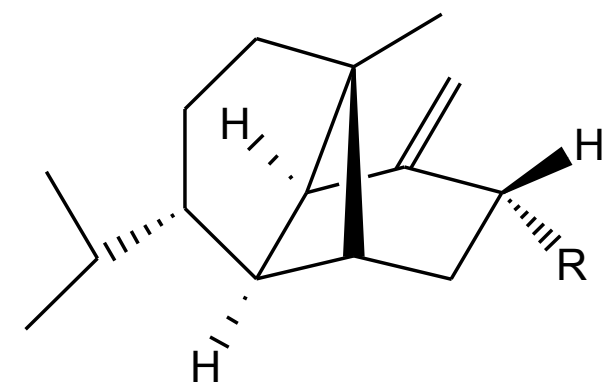

27: $\mathrm{R}=\mathrm{OCHO}$

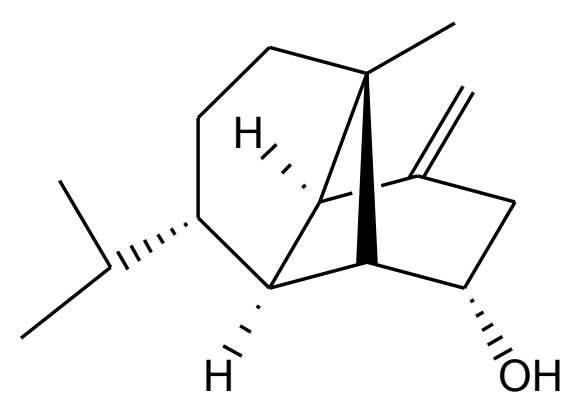

29

\subsubsection{Germacrane-Type Sesquiterpenoids}

Table 6 summarizes three germacrane-type sesquiterpenoids (30-32) evaluated for in vitro anti-inflammatory activity in literature published from 2008 to 2012. The corresponding chemical structures are reported in Figure 6. 
Table 6. Chemical constituents of germacrane-type sesquiterpenoids from soft corals of Taiwan.

\begin{tabular}{ccccc}
\hline No. & Name & Sources & Activities * & Reference \\
\hline 30 & Lochmolin G & Sinularia lochmodes & & {$[17]$} \\
31 & Menelloide D & Menella sp. & E & {$[19]$} \\
32 & Menelloide E & Menella sp. & & {$[20]$} \\
\hline
\end{tabular}

* Inhibition of elastase (E).

Figure 6. The structures of germacrane-type sesquiterpenoids (30-32).

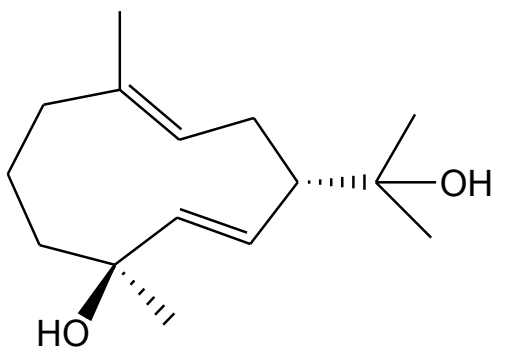

30

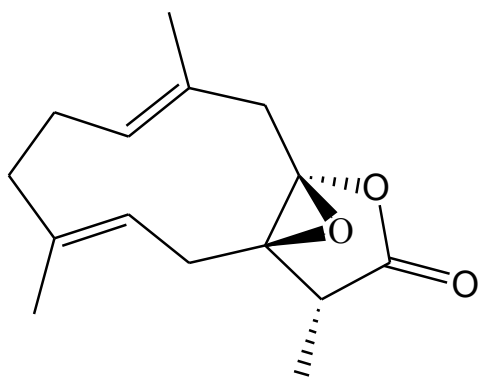

31

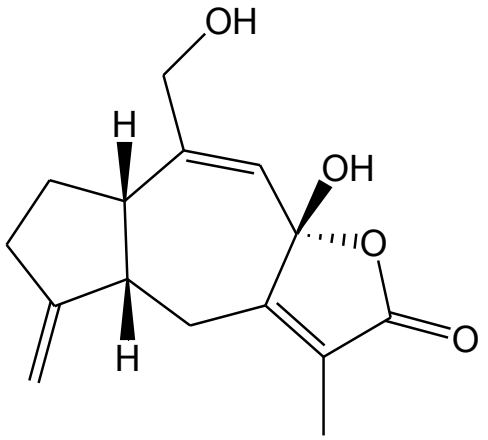

32

\subsubsection{Other-Type Sesquiterpenoids}

Table 7 summarizes six other-type sesquiterpenoids (33-38) evaluated for in vitro anti-inflammatory activity in literature published from 2008 to 2012. The corresponding chemical structures are reported in Figure 7.

Table 7. Chemical constituents of other-type sesquiterpenoids from soft corals of Taiwan.

\begin{tabular}{cllcc}
\hline No. & Name & Sources & Activities * & Reference \\
\hline $\mathbf{3 3}$ & Erectathiol & Nephthea erecta & I & {$[21]$} \\
$\mathbf{3 4}$ & Scabralin A & Sinularia scabra & I & {$[22]$} \\
35 & Leptocladol A & Sinularia leptoclados & & {$[23]$} \\
$\mathbf{3 6}$ & Paralemnolin D & Paralemnalia thyrsoides & {$[15]$} \\
$\mathbf{3 7}$ & 1-epi-Chabrolidione A & Sinularia leptoclados & & {$[23]$} \\
$\mathbf{3 8}$ & (-)-Hydroxylindestrenolide & Menella sp. & S & {$[24]$} \\
\hline \multicolumn{5}{c}{ * Inhibition of iNOS (I) and superoxide anion (S). }
\end{tabular}

Figure 7. The structures of other-type sesquiterpenoids (33-38).<smiles>CC(C)[C@H]1CC[C@@H](C)c2ccc(CS)cc21</smiles>

33<smiles>C=C1CC[C@H](C(C)C)[C@@]2(C)C=C(C)CC[C@]12O</smiles>

34

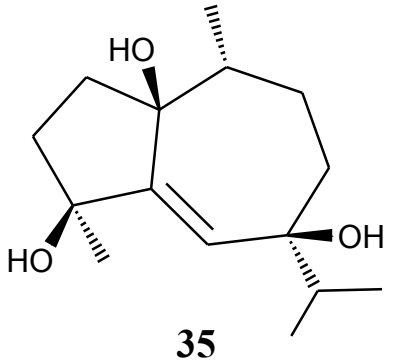

35 
Figure 7. Cont.

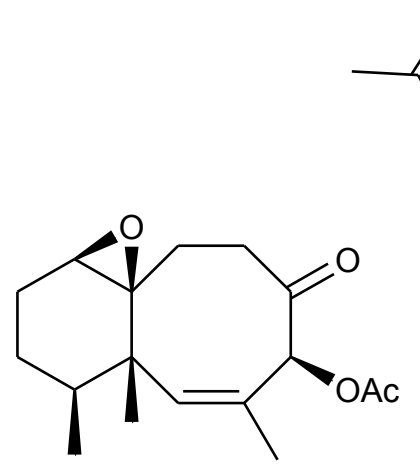

36

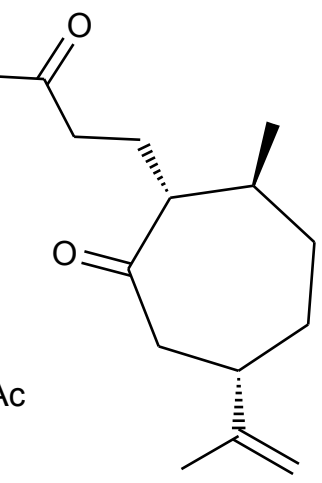

37

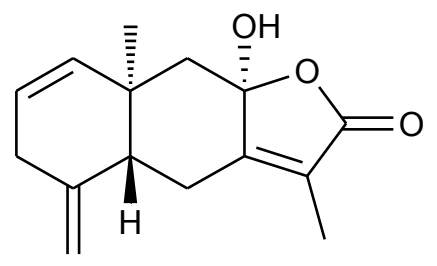

38

At a concentration of $10 \mu \mathrm{M}$, compounds 1-3, 13, 24, 28, 33 and 34 reduced LPS-induced expression of iNOS in murine macrophage cells [14-16,18,21,22]. Compounds 1, 2, 13, 17, 18, 21 and 28 suppressed LPS-induced expression of COX-2 in these cells [14-17]. At $10 \mu \mathrm{g} / \mathrm{mL}$, compound 38 was shown to slightly inhibit the generation of superoxide anion in FMLP/CB-stimulated human neutrophils, and compound $\mathbf{3 1}$ weakly inhibited the release of elastase by activated human neutrophils [19,24]. In addition, an inflammation animal model induced by intraplantar injection of carrageenan into rat hind paws was also used to evaluate in vivo anti-inflammatory activity of lemnalol (28). Intramuscular injection of $28(15 \mathrm{mg} / \mathrm{kg})$ significantly inhibited the carrageenan-induced rat paw edema and thermal hyperalgesia behavior. Moreover, lemnalol significantly suppressed the carrageenan-induced expression of iNOS and COX-2 in paw tissue of test rats. Post-intrathecal injection of lemnalol provided an antinociceptive effect in carrageenan-injected rats (1 and $5 \mu \mathrm{g})$ [25]. $\Delta^{9(12)}$-capnellene- $8 \beta, 10 \alpha$-diol $(\mathrm{GB} 9, \mathbf{1})$ and its acetylated derivative, $8 \alpha$-acetoxy- $\Delta^{9(12)}$-capnellene-10 $\alpha$-ol $(\mathrm{GB} 10,2)$ were reported to inhibit the expression of iNOS and COX-2 in BV2 cells post-stimulation by IFN- $\gamma$.

Intraperitoneal administration of GB9 reduced CCI-induced thermal hyperalgesia, suppressed microglial cells activation and COX-2 upregulation in the dorsal horn of the lumbar spinal cord, ipsilateral to the injury. Also, intrathecal administration of GB9 and GB10 suppressed activities of CCl-induced nociceptive sensitization and thermal hyperalgesia [26]. The above findings suggest that some of these compounds may warrant systematic investigation for future development as immune-modifiers.

\subsection{Diterpenoids}

\subsubsection{Cembrane-Based Diterpenoids}

Table 8 summarizes 92 cembrane-based diterpenoids (39-130) evaluated for in vitro anti-inflammatory activity in literature published from 2008 to 2012. The corresponding chemical structures are reported in Figure 8. 
Table 8. Chemical constituents of cembrane-based diterpenoids from soft corals of Taiwan.

\begin{tabular}{|c|c|c|c|c|}
\hline & Name & Sources & Activities * & Reference \\
\hline 39 & Gibberosene B & Sinularia gibberosa & $\mathrm{I}, \mathrm{C}$ & {$[27]$} \\
\hline 40 & (+)-11,12-Epoxysarcophytol A & Sinularia gibberosa & & {$[27]$} \\
\hline 41 & Grandilobatin B & Sinularia grandilobata & & [28] \\
\hline 42 & Grandilobatin D & Sinularia grandilobata & I & {$[28]$} \\
\hline 43 & Durumolide A & Lobophytum durum & $\mathrm{I}, \mathrm{C}$ & [29] \\
\hline 44 & 13S-Hydroxylobolide & Lobophytum durum & $\mathrm{I}, \mathrm{C}$ & [29] \\
\hline 45 & 13R-Hydroxylobolide & Lobophytum durum & I & [29] \\
\hline 46 & Deacetyl-13-hydroxylobolide & Lobophytum durum & $\mathrm{I}, \mathrm{C}$ & [27] \\
\hline 47 & $\begin{array}{l}(7 E, 11 E) \text {-13,18-Dihydroxy-3,4-epoxy- } \\
7,11,15(17) \text {-cembratrien-16,14-olide }\end{array}$ & Lobophytum durum & $\mathrm{I}, \mathrm{C}$ & {$[27]$} \\
\hline 48 & Durumolide B & Lobophytum durum & I & {$[28]$} \\
\hline 49 & $\begin{array}{l}(3 E, 7 E, 11 E)-18 \text {-Acetoxy-3,7,11,15(17)- } \\
\text { cembratetraen-16,14-olide }\end{array}$ & Lobophytum durum & $\mathrm{I}, \mathrm{C}$ & {$[28]$} \\
\hline 50 & Durumolide C & Lobophytum durum & $\mathrm{I}, \mathrm{C}$ & [29] \\
\hline 51 & Durumolide D & Lobophytum durum & $\mathrm{I}$ & [29] \\
\hline 52 & Durumolide E & Lobophytum durum & $\mathrm{I}$ & [29] \\
\hline 53 & Granosolide C & Sinularia granosa & & {$[30]$} \\
\hline 54 & Querciformolide E & Sinularia querciformis & I & {$[30]$} \\
\hline 55 & Granosolide D & Sinularia granosa & $\mathrm{I}$ & {$[30]$} \\
\hline 56 & Flexibilisolide A & Sinularia granosa & I & {$[30]$} \\
\hline 57 & Flexilarin & Sinularia granosa & I & {$[30]$} \\
\hline 58 & Sinulariolide & Sinularia granosa & I & [30] \\
\hline 59 & Sinulaflexiolide E & Sinularia granosa & & {$[30]$} \\
\hline 60 & Crassumolide A & Lobophytum crassum & $\mathrm{I}, \mathrm{C}$ & {$[31]$} \\
\hline 61 & Crassumolide B & Lobophytum crassum & $\mathrm{I}$ & {$[31]$} \\
\hline 62 & Crassumolide $\mathrm{C}$ & Lobophytum crassum & $\mathrm{I}, \mathrm{C}$ & {$[31]$} \\
\hline 63 & Crassumolide F & Lobophytum crassum & I & [31] \\
\hline 64 & Lobohedleolide & Lobophytum crassum & $\mathrm{I}, \mathrm{C}$ & [31] \\
\hline 65 & 17-Dimethylaminolobohedleolide & Lobophytum crassum & I & {$[31]$} \\
\hline 66 & Sinulariol A & Lobophytum crassum & $\mathrm{I}, \mathrm{C}$ & {$[31]$} \\
\hline 67 & Dentivulatolide & Lobophytum crassum & $\mathrm{I}, \mathrm{C}$ & {$[31]$} \\
\hline 68 & Durumhemiketalolide A & Lobophytum durum & $\mathrm{I}, \mathrm{C}$ & {$[32]$} \\
\hline 69 & Durumhemiketalolide B & Lobophytum durum & $\mathrm{I}$ & {$[32]$} \\
\hline 70 & Durumhemiketalolide C & Lobophytum durum & $\mathrm{I}, \mathrm{C}$ & {$[32]$} \\
\hline 71 & Durumolide F & Lobophytum durum & $\mathrm{I}, \mathrm{C}$ & {$[33]$} \\
\hline 72 & Durumolide G & Lobophytum durum & I & {$[33]$} \\
\hline 73 & Durumolide H & Lobophytum durum & I & {$[33]$} \\
\hline 74 & Durumolide I & Lobophytum durum & I & {$[33]$} \\
\hline 75 & Durumolide J & Lobophytum durum & I & {$[33]$} \\
\hline 76 & Sinularolide D & Lobophytum durum & I & {$[33]$} \\
\hline 77 & Durumolide K & Lobophytum durum & $\mathrm{I}, \mathrm{C}$ & {$[33]$} \\
\hline 78 & Durumolide L & Lobophytum durum & $\mathrm{I}$ & {$[33]$} \\
\hline
\end{tabular}


Table 8. Cont.

\begin{tabular}{|c|c|c|c|c|}
\hline 79 & Sarcocrassocolide A & Sarcophyton crassocaule & I & {$[34]$} \\
\hline 80 & Sarcocrassocolide C & Sarcophyton crassocaule & $\mathrm{I}$ & [34] \\
\hline 81 & Sarcocrassocolide B & Sarcophyton crassocaule & I & {$[34]$} \\
\hline 82 & Sarcocrassocolide D & Sarcophyton crassocaule & I & {$[34]$} \\
\hline 83 & Sarcocrassocolide E & Sarcophyton crassocaule & I & [34] \\
\hline 84 & Sarcocrassolide & Sarcophyton crassocaule & $\mathrm{I}, \mathrm{C}$ & {$[34]$} \\
\hline 85 & Sinularolide & Sarcophyton crassocaule & I & {$[34]$} \\
\hline 86 & 13-Acetoxysarcocrassolide & Sarcophyton crassocaule & I & {$[34]$} \\
\hline 87 & Thioflexibilolide A & Sinularia flexibilis & $\mathrm{I}, \mathrm{C}$ & {$[35]$} \\
\hline 88 & Triangulene A & Sinularia triangular & & {$[36]$} \\
\hline 89 & Triangulene B & Sinularia triangular & & {$[36]$} \\
\hline 90 & Sinularin & Sinularia triangular & $\mathrm{I}$ & {$[36]$} \\
\hline 91 & Dihydrosinularin & Sinularia triangular & $\mathrm{I}, \mathrm{C}$ & {$[36]$} \\
\hline 92 & $(-) 14-D e o x y c r a s s i n$ & Sinularia triangular & $\mathrm{I}, \mathrm{C}$ & {$[36]$} \\
\hline 93 & Sarcocrassocolide F & Sarcophyton crassocaule & I & {$[37]$} \\
\hline 94 & Sarcocrassocolide G & Sarcophyton crassocaule & I & {$[37]$} \\
\hline 95 & Sarcocrassocolide H & Sarcophyton crassocaule & I & {$[37]$} \\
\hline 96 & Sarcocrassocolide I & Sarcophyton crassocaule & $\mathrm{I}, \mathrm{C}$ & {$[37]$} \\
\hline 97 & Sarcocrassocolide J & Sarcophyton crassocaule & I & {$[37]$} \\
\hline 98 & Sarcocrassocolide K & Sarcophyton crassocaule & $\mathrm{I}$ & {$[37]$} \\
\hline 99 & Sarcocrassocolide L & Sarcophyton crassocaule & $\mathrm{I}$ & {$[37]$} \\
\hline 100 & Sarcophytolin A & Lobophytum sarcophytoides & $\mathrm{I}$ & {$[38]$} \\
\hline 101 & Sarcophytolin B & Lobophytum sarcophytoides & I & {$[38]$} \\
\hline 102 & Sarcophytolin C & Lobophytum sarcophytoides & & {$[38]$} \\
\hline 103 & Sarcophytolin D & Lobophytum sarcophytoides & I & {$[38]$} \\
\hline 104 & 11-Dehydrosinulariolide & Sinularia discrepans & $\mathrm{I}, \mathrm{C}$ & {$[39]$} \\
\hline 105 & 11-epi-Sinulariolide acetate & Sinularia discrepans & $\mathrm{I}, \mathrm{C}$ & [39] \\
\hline 106 & Crassumolide G & Lobophytum crassum & I & {$[40]$} \\
\hline 107 & Crassumolide $\mathrm{H}$ & Lobophytum crassum & I & {$[40]$} \\
\hline 108 & Crassumolide I & Lobophytum crassum & I & {$[40]$} \\
\hline 109 & Crassarine A & Sinularia crassa & & [41] \\
\hline 110 & Crassarine B & Sinularia crassa & & [41] \\
\hline 111 & Crassarine $\mathrm{C}$ & Sinularia crassa & & [41] \\
\hline 112 & Crassarine D & Sinularia crassa & & [41] \\
\hline 113 & Crassarine E & Sinularia crassa & & [41] \\
\hline 114 & Crassarine F & Sinularia crassa & $\mathrm{C}$ & {$[41]$} \\
\hline 115 & Crassarine $\mathrm{G}$ & Sinularia crassa & & [41] \\
\hline 116 & Crassarine $\mathrm{H}$ & Sinularia crassa & I & [41] \\
\hline 117 & Sarcocrassocolide M & Sarcophyton crassocaule & $\mathrm{I}$ & {$[42]$} \\
\hline 118 & Sarcocrassocolide N & Sarcophyton crassocaule & $\mathrm{I}$ & {$[42]$} \\
\hline 119 & Sarcocrassocolide O & Sarcophyton crassocaule & I & {$[42]$} \\
\hline 120 & Culobophylin A & Lobophytum crassum & & [43] \\
\hline 121 & Culobophylin B & Lobophytum crassum & & {$[43]$} \\
\hline 122 & Culobophylin C & Lobophytum crassum & & {$[43]$} \\
\hline 123 & Lobophylin B & Lobophytum crassum & & [43] \\
\hline
\end{tabular}


Table 8. Cont.

\begin{tabular}{|c|c|c|c|c|}
\hline 124 & Lobophylin A & Lobophytum crassum & & {$[43]$} \\
\hline 125 & Lobocrassin A & Lobophytum crassum & & [44] \\
\hline 126 & Lobocrassin B & Lobophytum crassum & $\mathrm{S}, \mathrm{E}$ & [44] \\
\hline 127 & Lobocrassin $\mathrm{C}$ & Lobophytum crassum & & {$[44]$} \\
\hline 128 & Lobocrassin D & Lobophytum crassum & & [44] \\
\hline 129 & Lobocrassin E & Lobophytum crassum & & {$[44]$} \\
\hline 130 & Lobocrassin F & Lobophytum crassum & $\mathrm{E}$ & {$[20]$} \\
\hline
\end{tabular}

* Inhibition of iNOS (I), COX-2 (C), superoxide anion (S) and elastase (E).

Figure 8. The structures of cembrane-based diterpenoids (39-130).

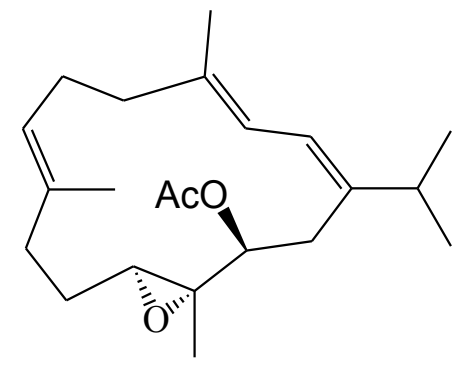

39

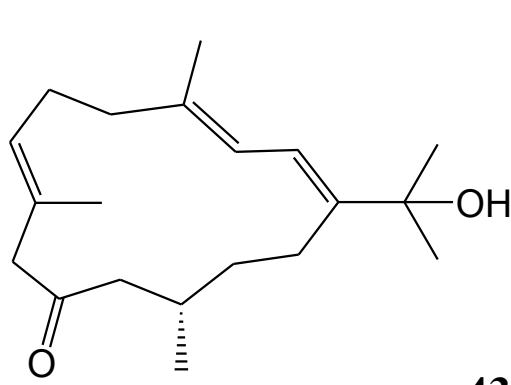

42

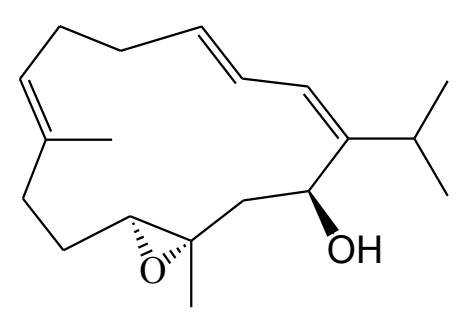

40

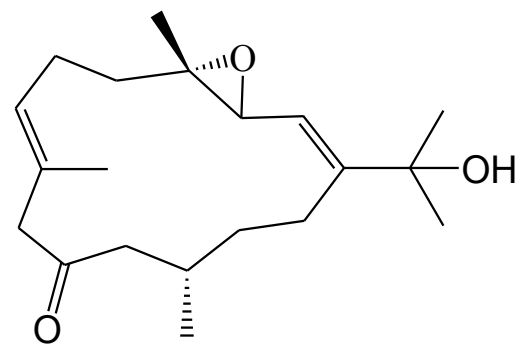

41
43: $\mathrm{R}_{1}=\mathrm{OAc}, \mathrm{R}_{2}=(R)-\mathrm{OH}, \mathrm{R}_{3}=\mathrm{OH}$

44: $\mathrm{R}_{1}=\mathrm{OAc}, \mathrm{R}=(S)-\mathrm{OH}, \mathrm{R}_{3}=\mathrm{H}$

45: $\mathrm{R}_{1}=\mathrm{OAc}, \mathrm{R}_{2}=(R)-\mathrm{OH}, \mathrm{R}_{3}=\mathrm{H}$

46: $\mathrm{R}_{1}=\mathrm{OH}, \mathrm{R}_{2}=(R)-\mathrm{OH}, \mathrm{R}_{3}=\mathrm{H}$

47: $\mathrm{R}_{1}=\mathrm{OH}, \mathrm{R}_{2}=(S)-\mathrm{OH}, \mathrm{R}_{3}=\mathrm{H}$<smiles></smiles>

48: $\mathrm{R}_{1}=\mathrm{OAc}, \mathrm{R}_{2}=\mathrm{OH}$

49: $\mathrm{R}_{1}=\mathrm{OAc}, \mathrm{R}_{2}=\mathrm{H}$

50: $\mathrm{R}_{1}=\mathrm{OH}, \mathrm{R}_{2}=(R)-\mathrm{OH}$

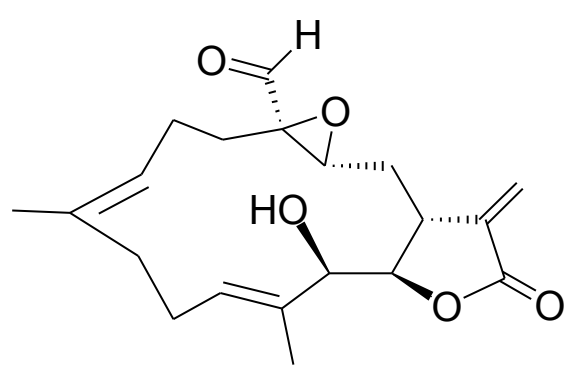

52

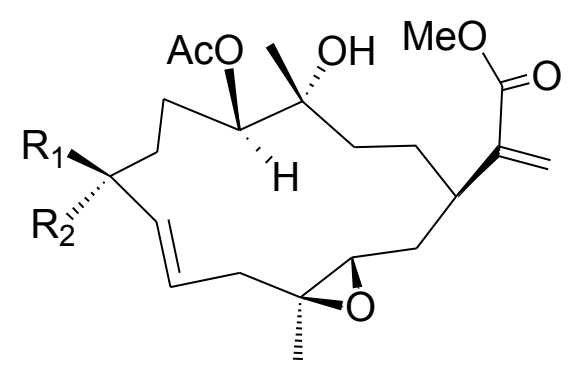

53: $\mathrm{R}_{1}=\mathrm{CH}_{3}, \mathrm{R}_{2}=\mathrm{OH}$

54: $\mathrm{R}_{1}=\mathrm{OH}, \mathrm{R}_{2}=\mathrm{CH}_{3}$ 
Figure 8. Cont.

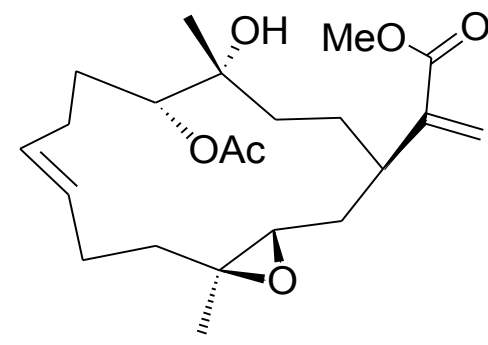

55

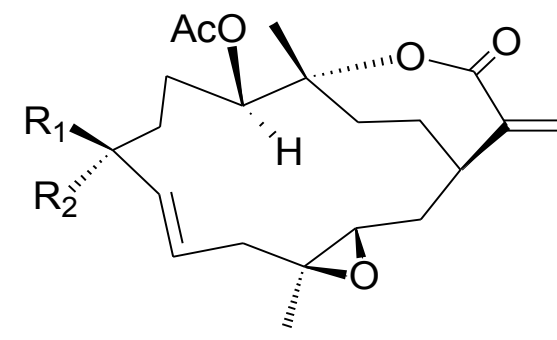

56: $\mathrm{R}_{1}=\mathrm{CH}_{3}, \mathrm{R}_{2}=\mathrm{OH}$ 57: $\mathrm{R}_{1}=\mathrm{OH}, \mathrm{R}_{2}=\mathrm{CH}_{3}$

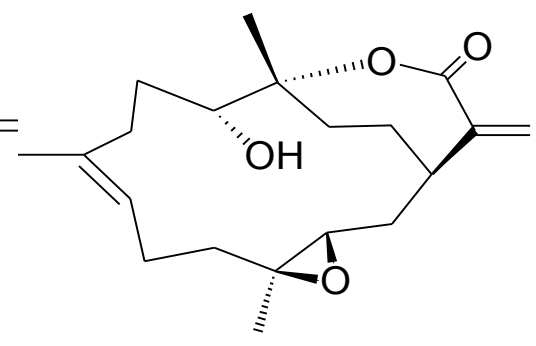

58

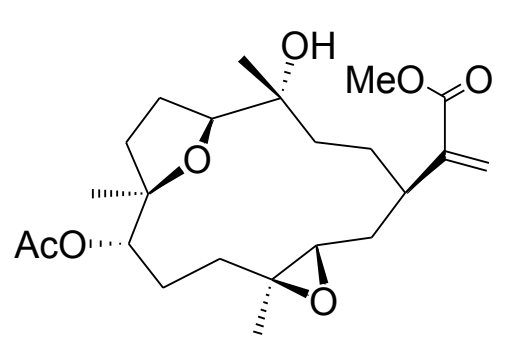

59

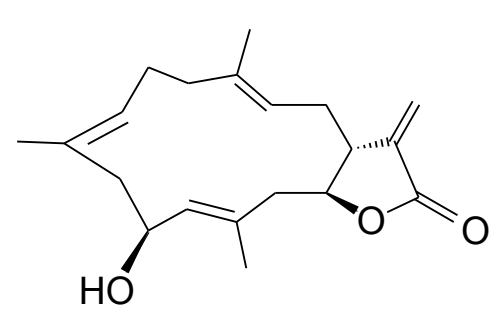

60

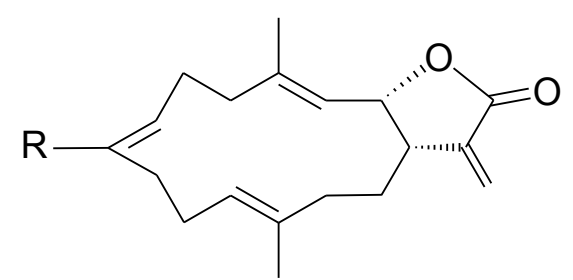

61: $\mathrm{R}=\mathrm{CH}_{2} \mathrm{OH}$

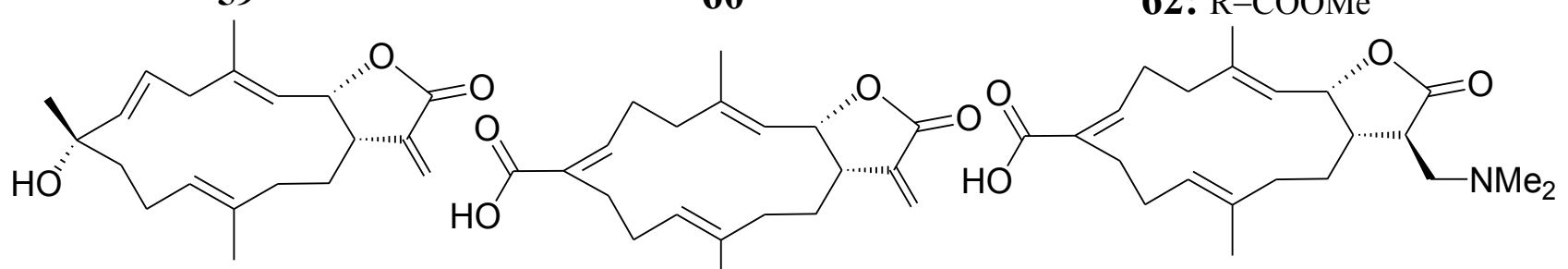

63

64

65

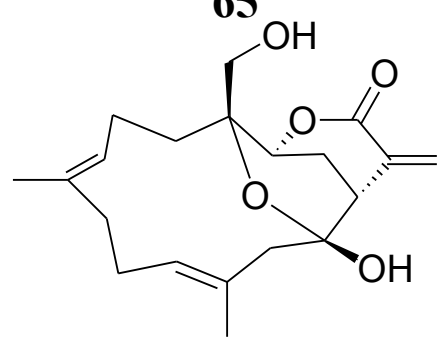

68

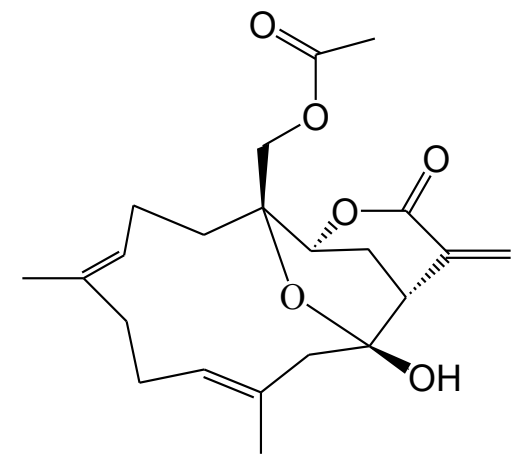

69
67

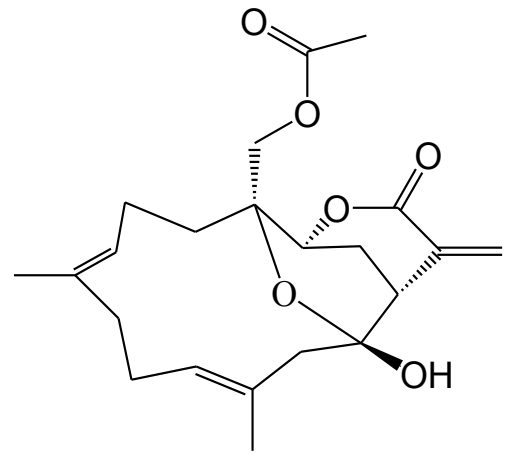

70

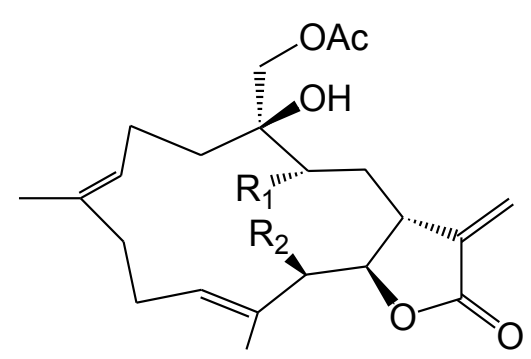

71: $\mathrm{R}_{1}=\mathrm{OH}, \mathrm{R}_{2}=\mathrm{H}$

72: $\mathrm{R}_{1}=\mathrm{OAc}, \mathrm{R}_{2}=\mathrm{OH}$

73: $\mathrm{R}_{1}=\mathrm{OAc}, \mathrm{R}_{2}=\mathrm{OAc}$ 
Figure 8. Cont.

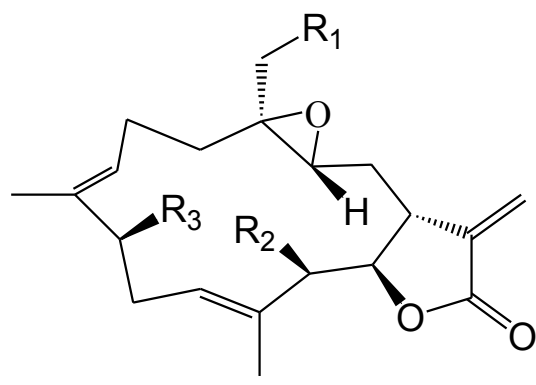

74: $\mathrm{R}_{1}=\mathrm{OAc}, \mathrm{R}_{2}=\mathrm{OAc}, \mathrm{R}_{3}=\mathrm{OH}$ 75: $\mathrm{R}_{1}=\mathrm{H}, \mathrm{R}_{2}=\mathrm{OH}, \mathrm{R}_{3}=\mathrm{H}$ 76: $\mathrm{R}_{1}=\mathrm{OH}, \mathrm{R}_{2}=\mathrm{H}, \mathrm{R}_{3}=\mathrm{H}$

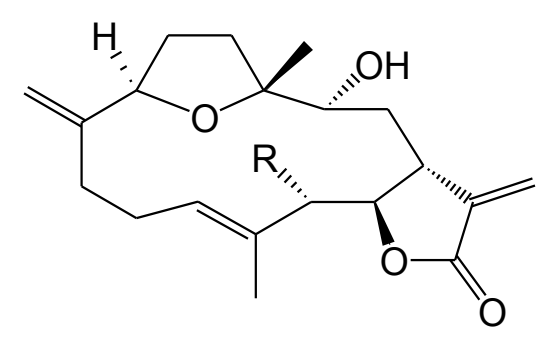

79: $\mathrm{R}=\mathrm{OAc}$

80: $\mathrm{R}=\mathrm{H}$

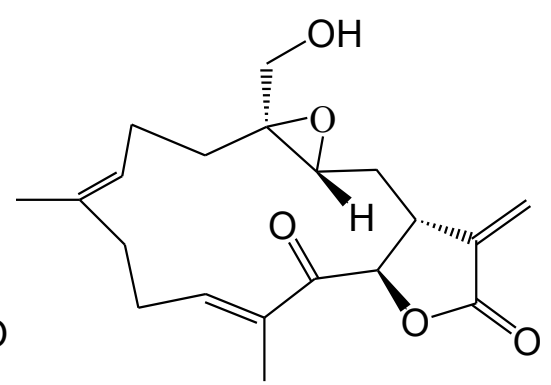

77

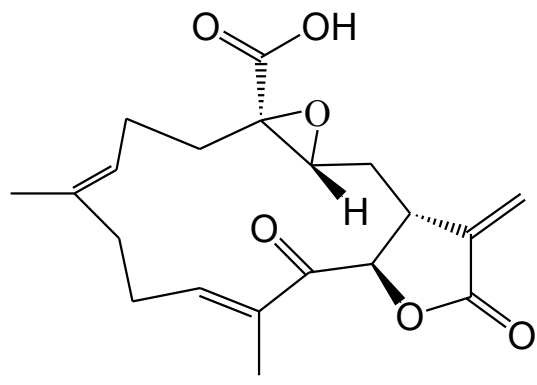

78

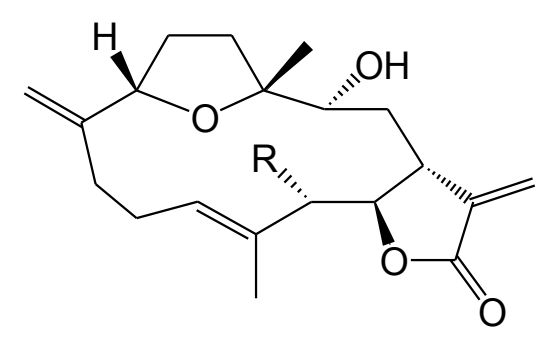

81: $\mathrm{R}=\mathrm{OAc}$

82: $\mathrm{R}=\mathrm{H}$

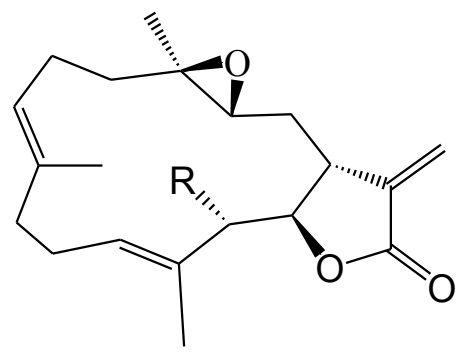

83: $\mathrm{R}=\mathrm{OCOE} t$

84: $\mathrm{R}=\mathrm{H}$

85: $\mathrm{R}=\mathrm{OH}$

86: $\mathrm{R}=\mathrm{OAc}$

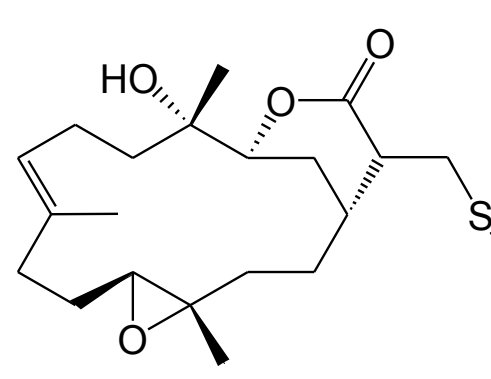

87

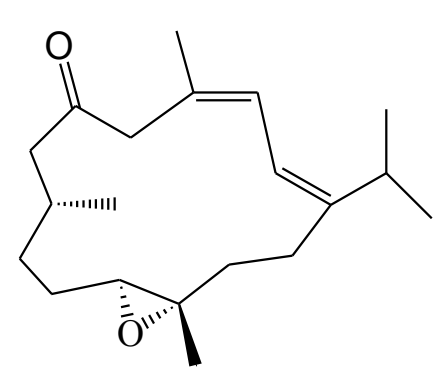

89

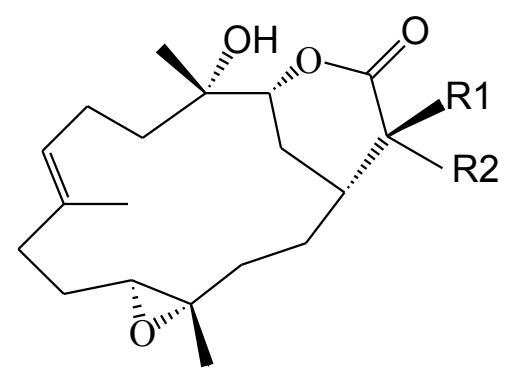

90: $\mathrm{R}_{1}=\mathrm{R}_{2}=\mathrm{CH}_{2}$

91: $\mathrm{R}_{1}=\mathrm{CH}_{3}, \mathrm{R}_{2}=\mathrm{H}$

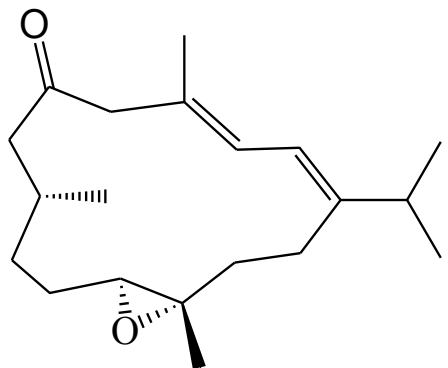

88

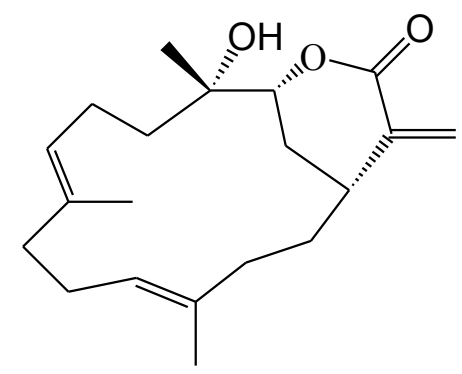

92 
Figure 8. Cont.

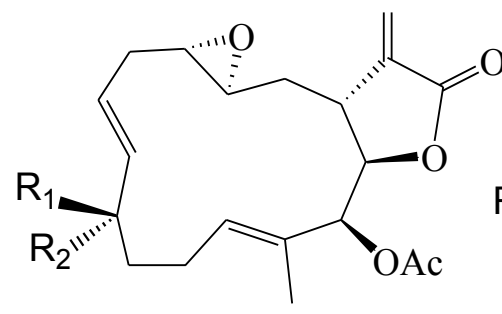

93: $\mathrm{R}_{1}=\mathrm{CH}_{3}, \mathrm{R}_{2}=\mathrm{OOH}$

94: $\mathrm{R}_{1}=\mathrm{OOH}, \mathrm{R}_{2}=\mathrm{CH}_{3}$

95: $\mathrm{R}_{1}=\mathrm{CH}_{3}, \mathrm{R}_{2}=\mathrm{OH}$

96: $\mathrm{R}_{1}=\mathrm{OH}, \mathrm{R}_{2}=\mathrm{CH}_{3}$

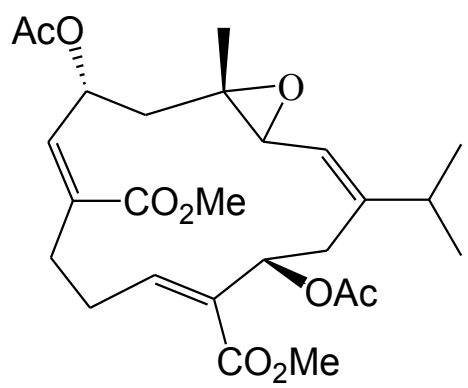

102

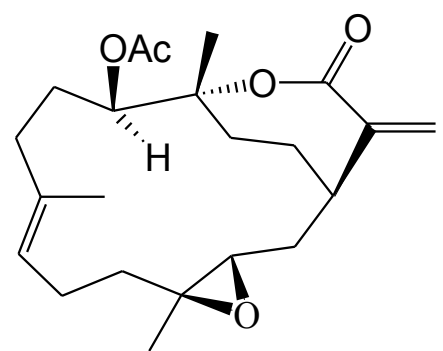

105

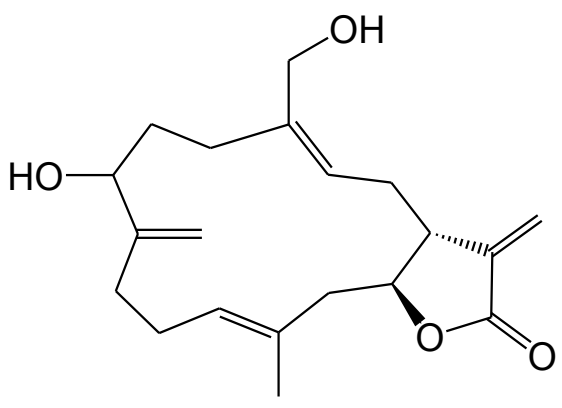

108

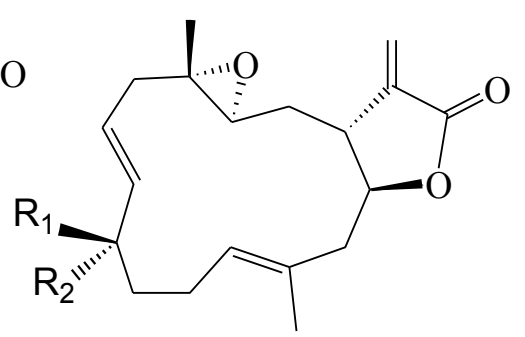

97: $\mathrm{R}_{1}=\mathrm{CH}_{3}, \mathrm{R}_{2}=\mathrm{OOH}$

98: $\mathrm{R}_{1}=\mathrm{CH}_{3}, \mathrm{R}_{2}=\mathrm{OH}$

99: $\mathrm{R}_{1}=\mathrm{OH}, \mathrm{R}_{2}=\mathrm{CH}_{3}$

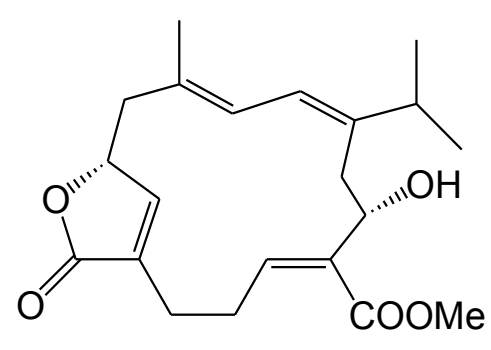

103

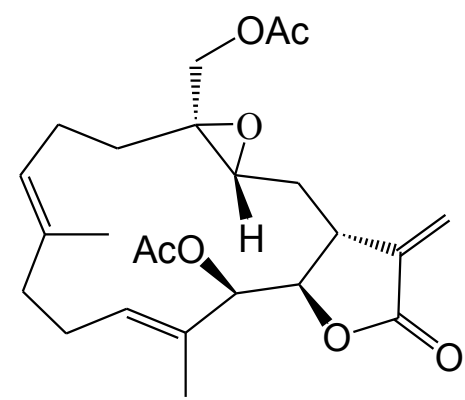

106

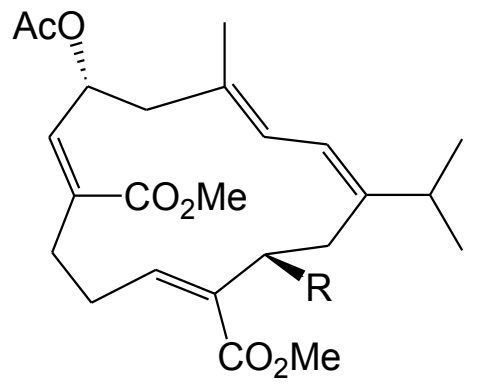

100: $\mathrm{R}=\mathrm{OH}$

101: $\mathrm{R}=\mathrm{OAc}$

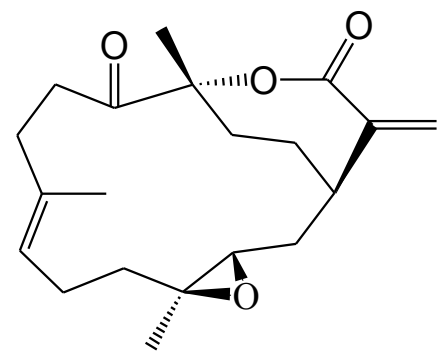

104

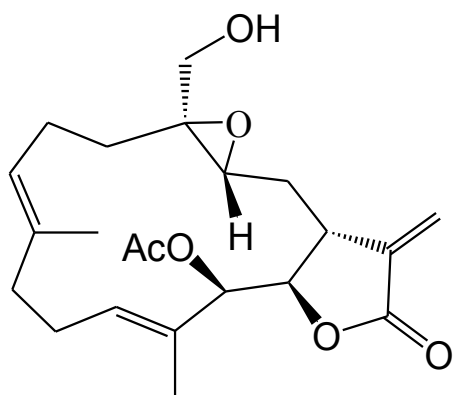

107

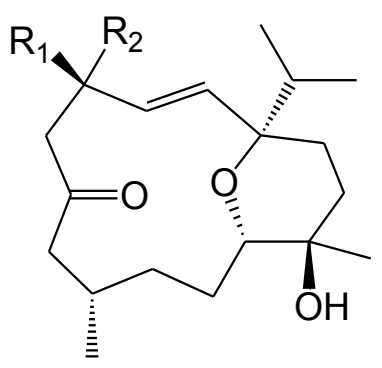

112: $\mathrm{R}_{1}=\mathrm{OH}, \mathrm{R}_{2}=\mathrm{CH}_{3}$

113: $\mathrm{R}_{1}=\mathrm{CH}_{3}, \mathrm{R}_{2}=\mathrm{OH}$

109: $\mathrm{R}_{1}=\mathrm{CH}_{3}, \mathrm{R}_{2}=\mathrm{OH}, \mathrm{R}_{3}=\mathrm{H}$

110: $\mathrm{R}_{1}=\mathrm{OH}, \mathrm{R}_{2}=\mathrm{CH}_{3}, \mathrm{R}_{3}=\mathrm{Ac}$

111: $\mathrm{R}_{1}=\mathrm{OH}, \mathrm{R}_{2}=\mathrm{CH}_{3}, \mathrm{R}_{3}=\mathrm{CHO}$ 
Figure 8. Cont.

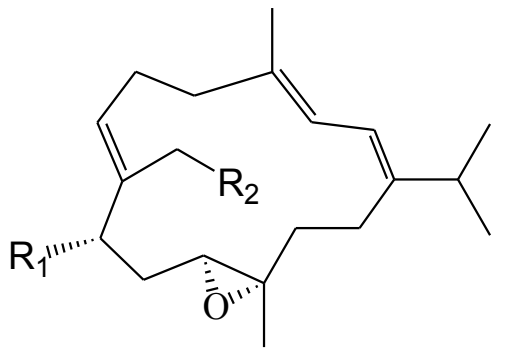

114: $\mathrm{R}_{1}=\mathrm{OH}, \mathrm{R}_{2}=\mathrm{H}$

115: $\mathrm{R}_{1}=\mathrm{H}, \mathrm{R}_{2}=\mathrm{OH}$

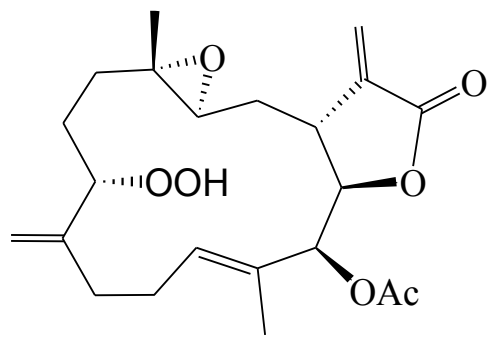

119

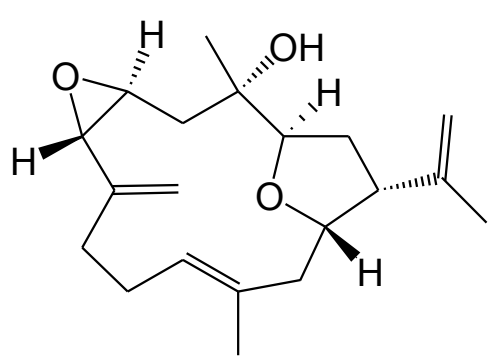

122

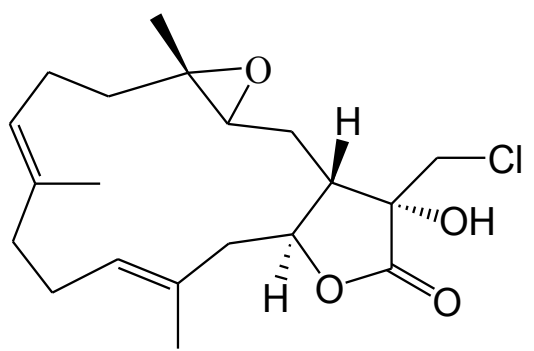

125

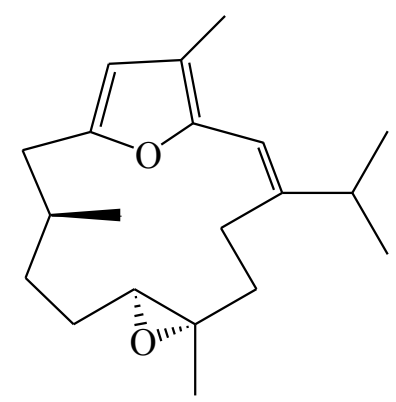

116

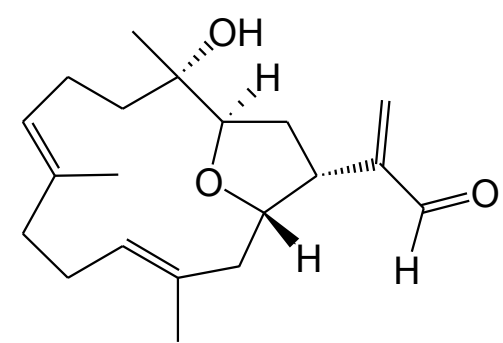

120

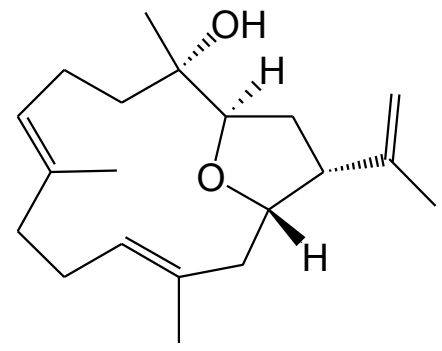

123

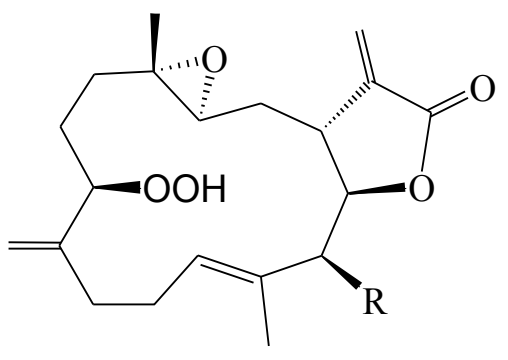

117: $\mathrm{R}=\mathrm{OAc}$

118: $\mathrm{R}=\mathrm{H}$

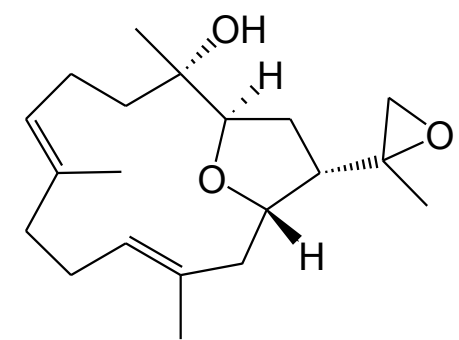

121

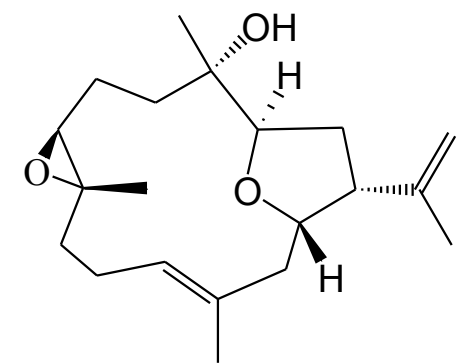

124

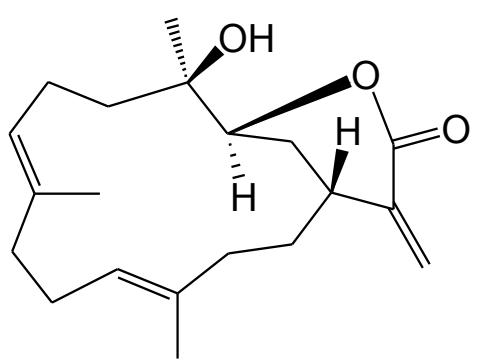

126

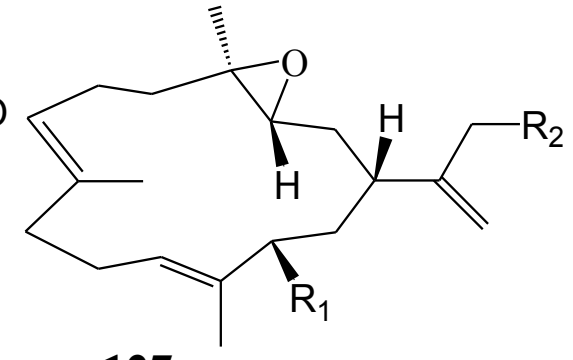

127: $\mathrm{R}_{1}=\mathrm{H}, \mathrm{R}_{2}=\mathrm{OH}$

128: $\mathrm{R}_{1}=\mathrm{H}, \mathrm{R}_{2}=\mathrm{OAc}$

129: $R_{1}=O H, R_{2}=H$

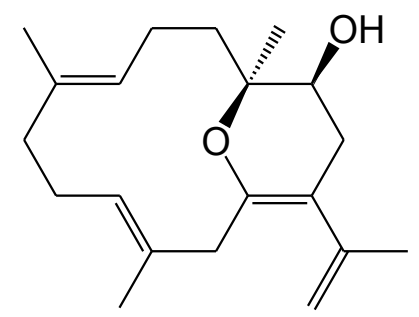


At the concentration of $10 \mu \mathrm{M}$, compounds 39, 42-52, 54-58, 60-87, 90-101, 103-108 and 116-119 reduced LPS-induced expression of iNOS in murine macrophage (RAW264.7) cells [27-42]. Compounds 39, 43, 44, 46, 47, 49, 50, 62, 64, 66-68, 70, 71, 77, 84, 87, 91, 92, 96, 104, 105 and 114 suppressed LPS-induced expression of COX-2 in these cells [27,29,31-37,39,41]. At $10 \mu \mathrm{g} / \mathrm{mL}$, compound 126 inhibited the generation of superoxide anion and the release of elastase in human neutrophils [44]. Compound 130 inhibited the release of elastase by activated human neutrophils [24]. For in vivo anti-inflammatory activities, subcutaneous (s.c.) administration of sinularin (90) $(80 \mathrm{mg} / \mathrm{kg})$ significantly inhibited carrageenan-induced nociceptive behaviors as well as carrageenan-induced activation of microglial and astrocyte, and the iNOS expression in the dorsal horn of the lumbar spinal cord [45]. Due to its promising anti-inflammatory profile, sinularin may warrant future exploration as a lead compound for immune-/inflammation-modulation.

\subsubsection{Eunicellin-Based Diterpenoids}

Table 9 summarizes 58 eunicellin-based diterpenoids (131-188) evaluated for in vitro anti-inflammatory activity in literature published from 2008 to 2012. The corresponding chemical structures are reported in Figure 9.

Table 9. Chemical constituents of eunicellin-based diterpenoids from soft corals of Taiwan.

\begin{tabular}{|c|c|c|c|c|}
\hline No. & Name & Sources & Activities * & Reference \\
\hline 131 & Simplexin A & Klyxum simplex & I & {$[46]$} \\
\hline 132 & Simplexin B & Klyxum simplex & & {$[46]$} \\
\hline 133 & Simplexin C & Klyxum simplex & & {$[46]$} \\
\hline 134 & Simplexin D & Klyxum simplex & I & {$[46]$} \\
\hline 135 & Simplexin E & Klyxum simplex & $\mathrm{I}, \mathrm{C}$ & {$[46]$} \\
\hline 136 & Simplexin F & Klyxum simplex & & {$[46]$} \\
\hline 137 & Simplexin I & Klyxum simplex & & [46] \\
\hline 138 & Klysimplexin I & Klyxum simplex & & {$[47]$} \\
\hline 139 & Klysimplexin J & Klyxum simplex & I & [47] \\
\hline 140 & Klysimplexin $\mathrm{K}$ & Klyxum simplex & I & [47] \\
\hline 141 & Klysimplexin L & Klyxum simplex & I & {$[47]$} \\
\hline 142 & Klysimplexin M & Klyxum simplex & I & {$[47]$} \\
\hline 143 & Klysimplexin N & Klyxum simplex & I & [47] \\
\hline 144 & Klysimplexin O & Klyxum simplex & & [47] \\
\hline 145 & Klysimplexin P & Klyxum simplex & & [47] \\
\hline 146 & Klysimplexin Q & Klyxum simplex & & {$[47]$} \\
\hline 147 & Klysimplexin R & Klyxum simplex & I & {$[47]$} \\
\hline 148 & Klysimplexin S & Klyxum simplex & $\mathrm{I}, \mathrm{C}$ & {$[47]$} \\
\hline 149 & Klysimplexin T & Klyxum simplex & & [47] \\
\hline 150 & Hirsutalin A & Cladiella hirsuta & & {$[48]$} \\
\hline 151 & Hirsutalin B & Cladiella hirsuta & $\mathrm{I}, \mathrm{C}$ & {$[48]$} \\
\hline 152 & Hirsutalin C & Cladiella hirsuta & I & {$[48]$} \\
\hline 153 & Hirsutalin D & Cladiella hirsuta & I & [48] \\
\hline 154 & Hirsutalin E & Cladiella hirsuta & & {$[48]$} \\
\hline 155 & Hirsutalin F & Cladiella hirsuta & & [48] \\
\hline
\end{tabular}


Table 9. Cont.

\begin{tabular}{|c|c|c|c|c|}
\hline 156 & Hirsutalin G & Cladiella hirsuta & & [48] \\
\hline 157 & Hirsutalin $\mathrm{H}$ & Cladiella hirsuta & I & [48] \\
\hline 158 & Klysimplexin sulfoxide A & Klyxum simplex & I & [49] \\
\hline 159 & Klysimplexin sulfoxide B & Klyxum simplex & I & [49] \\
\hline 160 & Klysimplexin sulfoxide C & Klyxum simplex & $\mathrm{I}, \mathrm{C}$ & [49] \\
\hline 161 & Lymollin A & Klyxum molle & & {$[50]$} \\
\hline 162 & Lymollin B & Klyxum molle & I & {$[50]$} \\
\hline 163 & Lymollin C & Klyxum molle & $\mathrm{I}, \mathrm{C}$ & {$[50]$} \\
\hline 164 & Lymollin D & Klyxum molle & $\mathrm{I}, \mathrm{C}$ & {$[50]$} \\
\hline 165 & Lymollin E & Klyxum molle & I & [50] \\
\hline 166 & Lymollin F & Klyxum molle & $\mathrm{I}, \mathrm{C}$ & {$[50]$} \\
\hline 167 & Lymollin G & Klyxum molle & $\mathrm{I}, \mathrm{C}$ & {$[50]$} \\
\hline 168 & Lymollin $\mathrm{H}$ & Klyxum molle & $\mathrm{I}, \mathrm{C}$ & {$[50]$} \\
\hline 169 & Krempfielin A & Cladiella krempfi & & [51] \\
\hline 170 & Krempfielin D & Cladiella krempfi & $\mathrm{I}$ & [51] \\
\hline 171 & Krempfielin B & Cladiella krempfi & $\mathrm{I}$ & [51] \\
\hline 172 & krempfielin $\mathrm{C}$ & Cladiella krempfi & $\mathrm{I}$ & [51] \\
\hline 173 & Litophynol B & Cladiella krempfi & I & [51] \\
\hline 174 & $\begin{array}{c}\left(1 R^{*}, 2 R^{*}, 3 R^{*}, 6 S^{*}, 7 S^{*}, 9 R^{*}, 10 R^{*}, 14 R^{*}\right) 3- \\
\text { Butanoyloxycladiell-11(17)-en-6,7-diol }\end{array}$ & Cladiella krempfi & I & {$[51]$} \\
\hline 175 & Klysimplexin U & Klyxum simplex & & {$[52]$} \\
\hline 176 & Klysimplexin V & Klyxum simplex & & {$[52]$} \\
\hline 177 & Klysimplexin W & Klyxum simplex & & {$[52]$} \\
\hline 178 & Klysimplexin X & Klyxum simplex & & {$[52]$} \\
\hline 179 & Cladieunicellin A & Cladiella sp. & $\mathrm{S}, \mathrm{E}$ & [53] \\
\hline 180 & Cladieunicellin C & Cladiella sp. & & [53] \\
\hline 181 & Cladieunicellin D & Cladiella sp. & & [53] \\
\hline 182 & Cladieunicellin E & Cladiella sp. & & {$[53]$} \\
\hline 183 & Cladieunicellin G & Cladiella sp. & $\mathrm{S}, \mathrm{E}$ & {$[54]$} \\
\hline 184 & 6-epi-Cladieunicellin F & Cladiella sp. & & [54] \\
\hline 185 & Cladieunicellin F & Cladiella sp. & $\mathrm{S}, \mathrm{E}$ & {$[54]$} \\
\hline 186 & (-)-Solenopodin C & Cladiella sp. & & {$[55]$} \\
\hline 187 & Cladielloide A & Cladiella sp. & & {$[56]$} \\
\hline 188 & Cladielloide B & Cladiella sp. & $\mathrm{S}, \mathrm{E}$ & [56] \\
\hline
\end{tabular}

* Inhibition of iNOS (I), COX-2 (C), superoxide anion (S) and elastase (E).

Figure 9. The structures of cembrane-based diterpenoids (131-188).

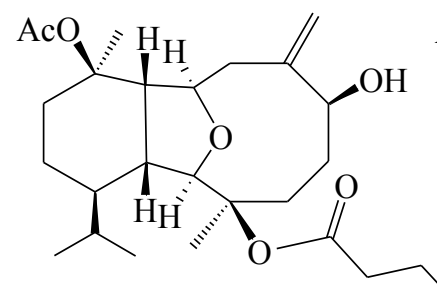

131

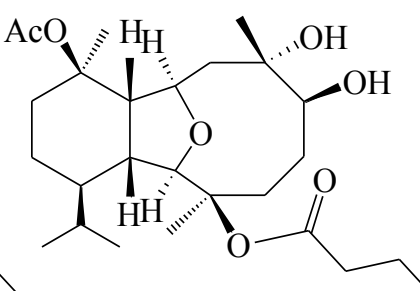

132
133: $\mathrm{R}_{1}=\mathrm{R}_{2}=\mathrm{Ac}, \mathrm{R}_{3}=\mathrm{COCH}_{2} \mathrm{CH}_{2} \mathrm{CH}_{3}$

134: $\mathrm{R}_{1}=\mathrm{COCH}_{2} \mathrm{CH}_{2} \mathrm{CH}_{3}, \mathrm{R}_{2}=\mathrm{Ac}, \mathrm{R}_{3}=\mathrm{COCH}_{2} \mathrm{CH}_{2} \mathrm{CH}_{3}$ 135: $\mathrm{R}_{1}=\mathrm{COCH}=\mathrm{CH}_{2}, \mathrm{R}_{2}=\mathrm{Ac}, \mathrm{R}_{3}=\mathrm{COCH}_{2} \mathrm{CH}_{2} \mathrm{CH}_{3}$ 136: $R_{1}=R_{2}=A c, R_{3}=H$ 
Figure 9. Cont.

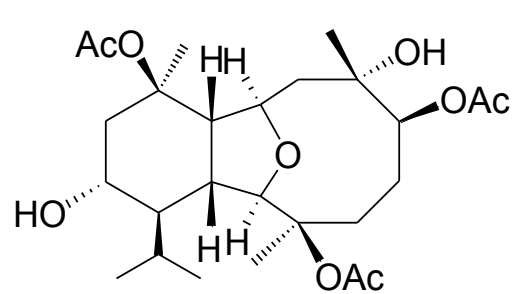

137

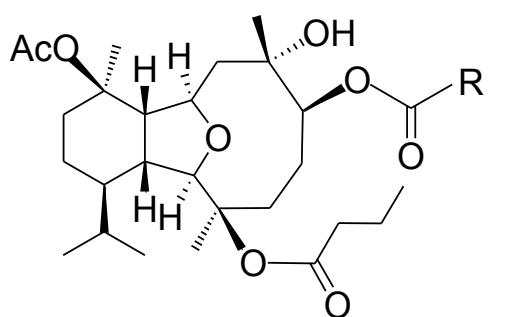

138: $\mathrm{R}=\left(\mathrm{CH}_{2}\right)_{12} \mathrm{CH}_{3}$

139: $\mathrm{R}=\left(\mathrm{CH}_{2}\right)_{14} \mathrm{CH}_{3}$

140: $\mathrm{R}=\left(\mathrm{CH}_{2}\right)_{16} \mathrm{CH}_{3}$

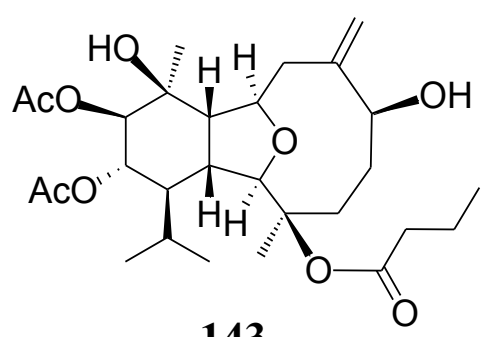

143

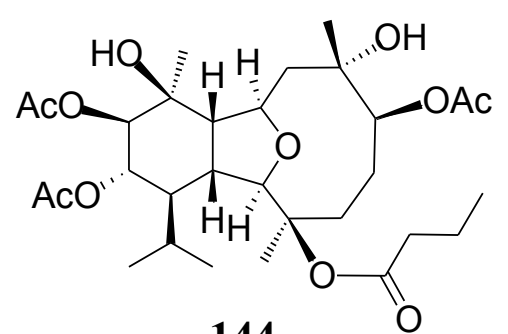

144

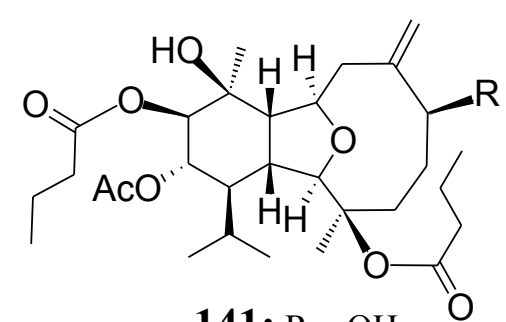

141: $\mathrm{R}=\mathrm{OH}$

142: $\mathrm{R}=\mathrm{OOH}$

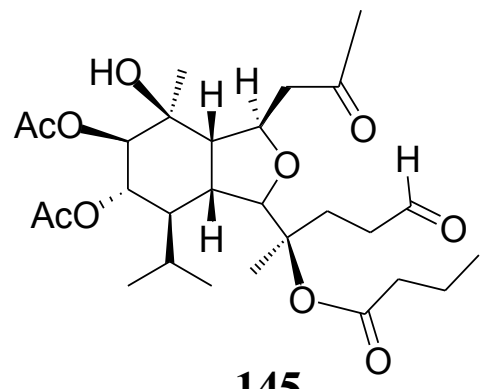

145

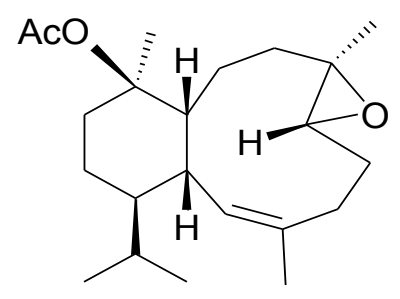

146

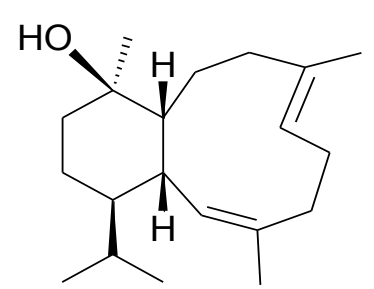

147

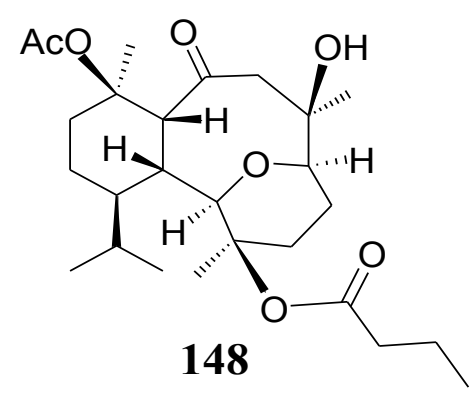

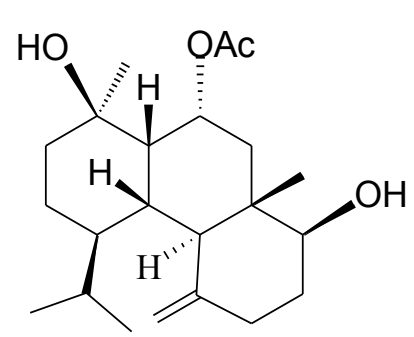

149

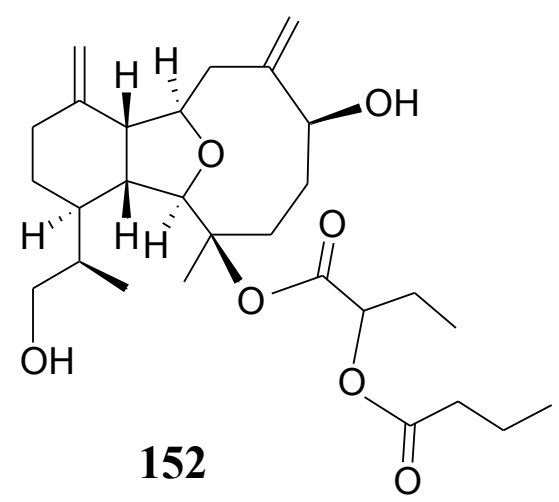

150

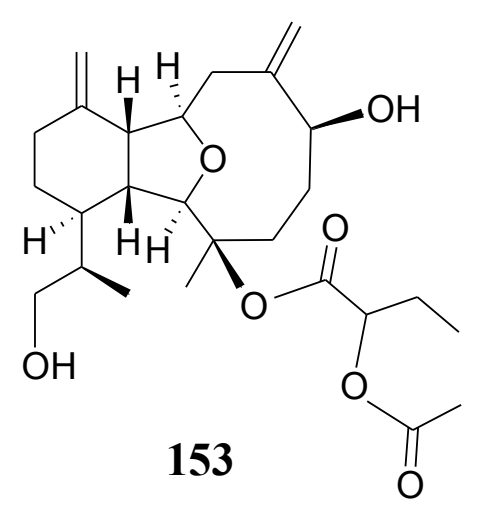

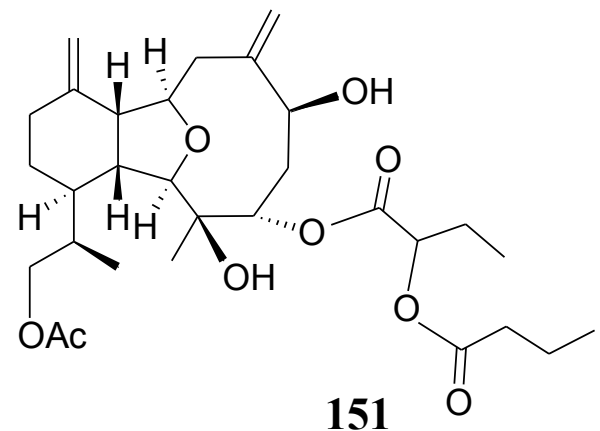

151

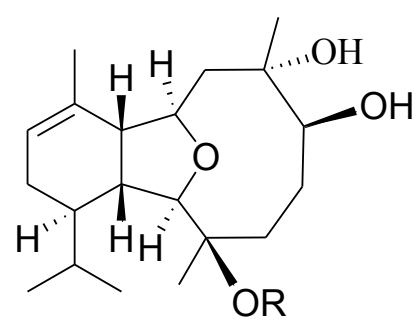

154: $\mathrm{R}=\mathrm{COCH}_{2} \mathrm{CH}_{2} \mathrm{CH}_{3}$ 
Figure 9. Cont.

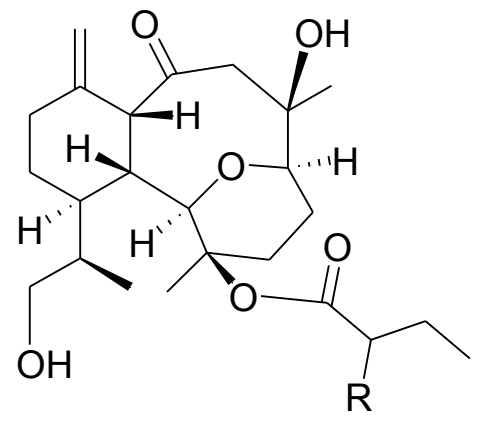

155: $\mathrm{R}=\mathrm{OCOCH}_{2} \mathrm{CH}_{2} \mathrm{CH}_{3}$

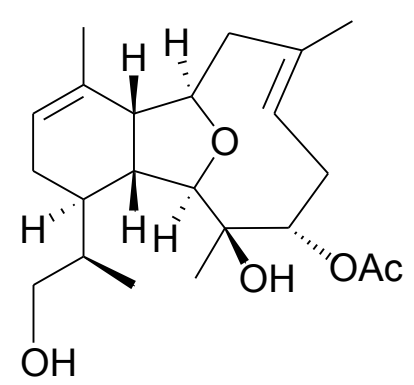

156

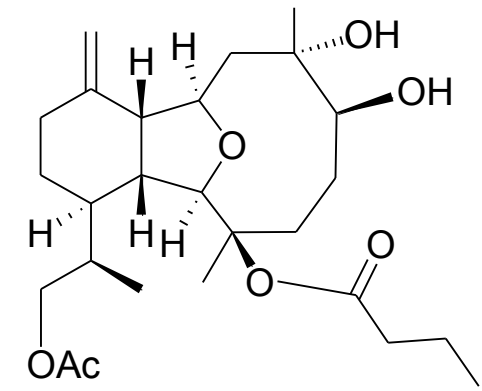

157

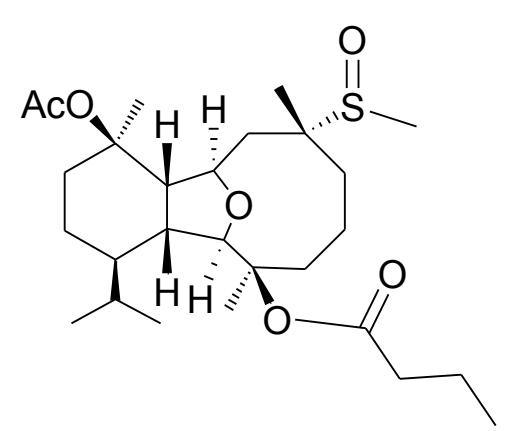

158

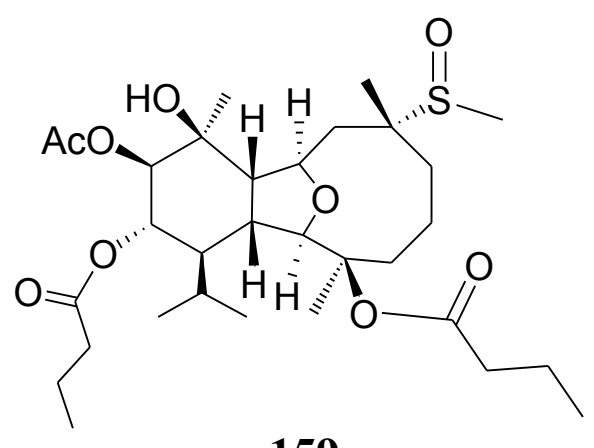

159

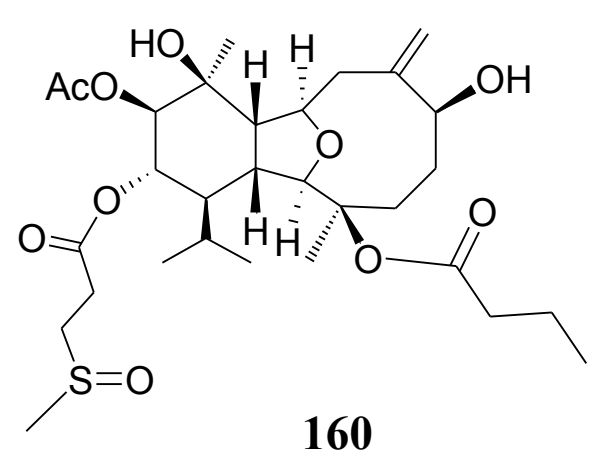

160

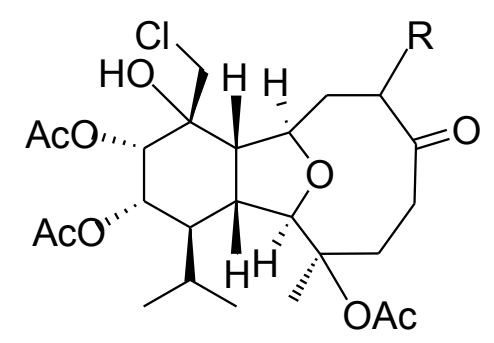

161: $\mathrm{R}=\beta-\mathrm{CH}_{3}$

162: $\mathrm{R}=\alpha-\mathrm{CH}_{3}$

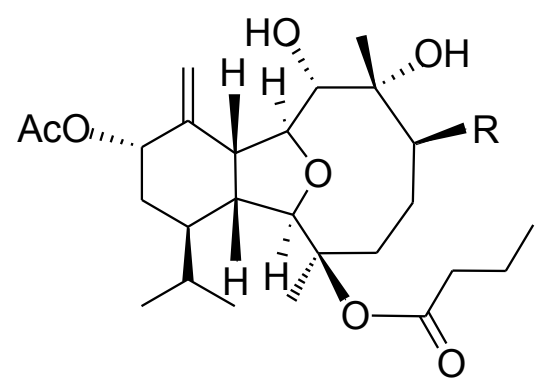

169: $\mathrm{R}=\mathrm{OH}$

170: $\mathrm{R}=\mathrm{OMe}$

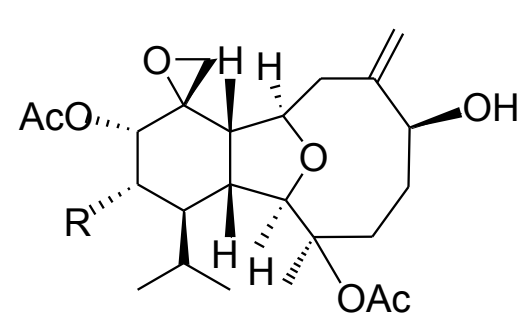

163: $\mathrm{R}=\mathrm{OAc}$

$164: R=H$

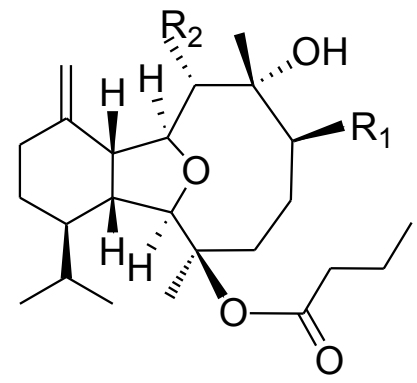

171: $\mathrm{R}_{1}=\mathrm{OMe}, \mathrm{R}_{2}=\mathrm{OH}$ 172: $\mathrm{R}_{1}=\mathrm{OAc}, \mathrm{R}_{2}=\mathrm{OH}$ 173: $\mathrm{R}_{1}=\mathrm{OH}, \mathrm{R}_{2}=\mathrm{OH}$ 174: $\mathrm{R}_{1}=\mathrm{OH}, \mathrm{R}_{2}=\mathrm{H}$

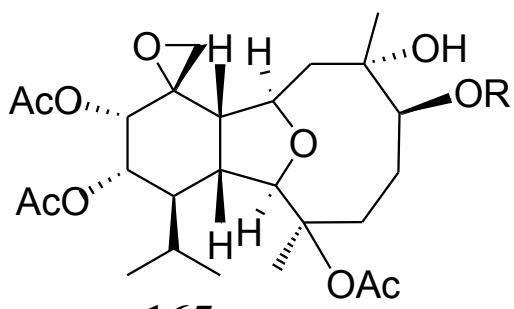

165: $\mathrm{R}=\mathrm{Ac}$

166: $\mathrm{R}=\mathrm{CH}_{3}\left(\mathrm{CH}_{2}\right)_{12} \mathrm{CO}$

167: $\mathrm{R}=\mathrm{CH}_{3}\left(\mathrm{CH}_{2}\right)_{14} \mathrm{CO}$

168: $\mathrm{R}=\mathrm{CHO}$

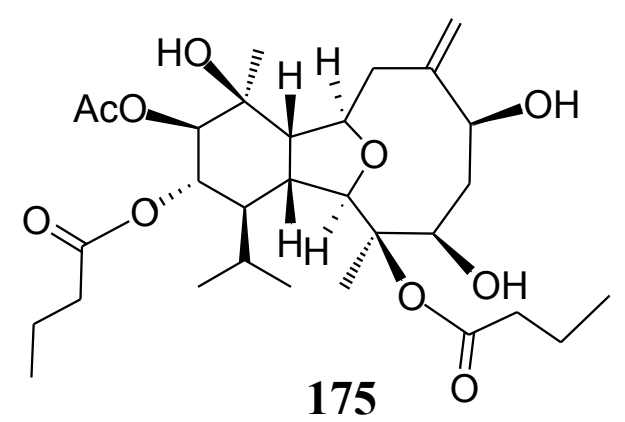

175 
Figure 9. Cont.

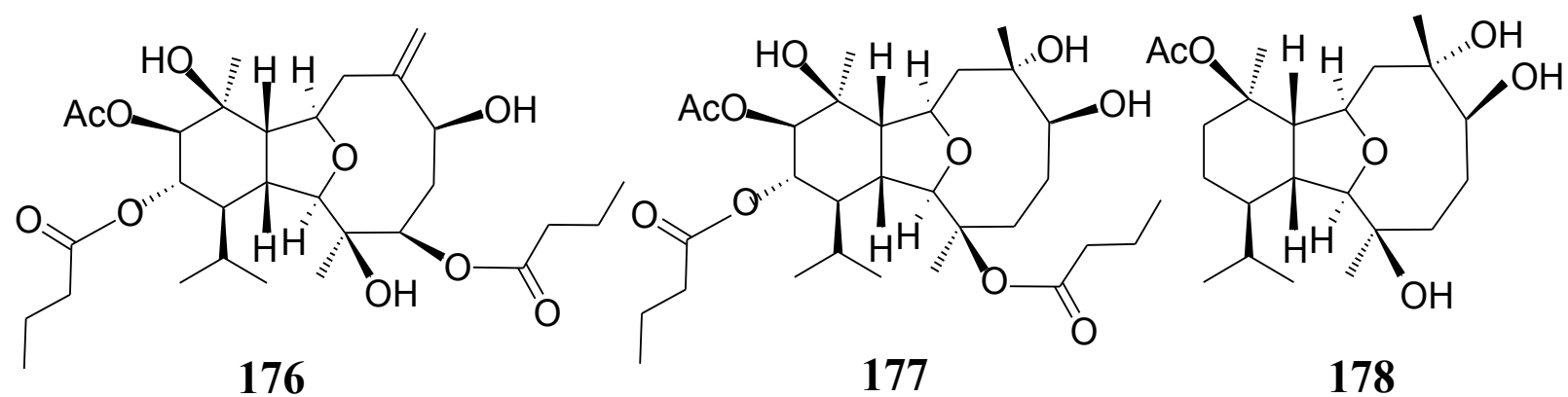

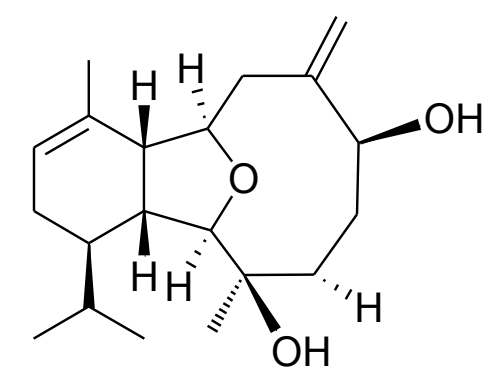

179

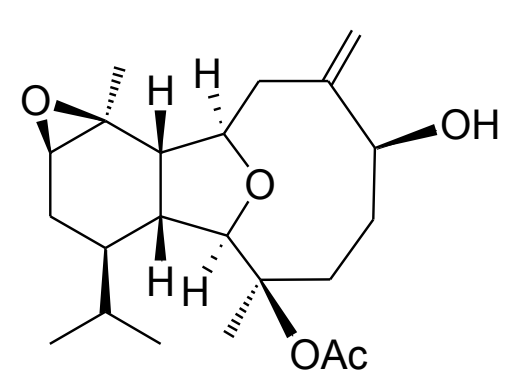

180

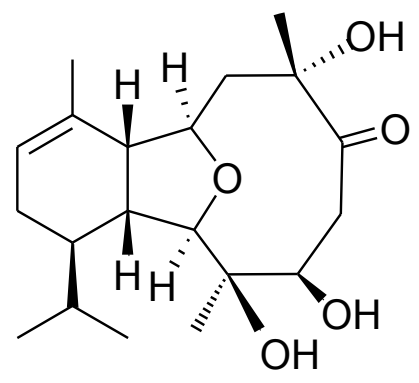

181

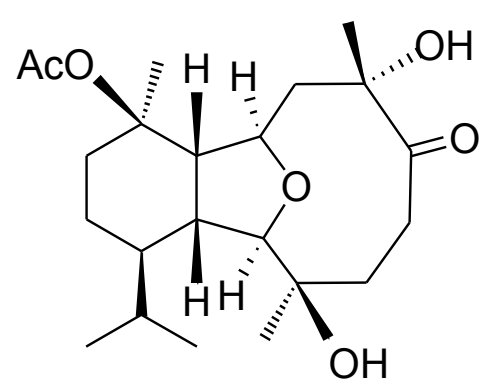

182

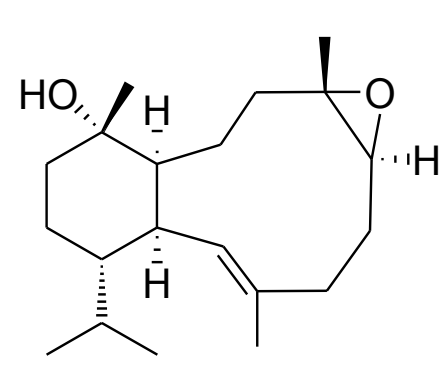

186

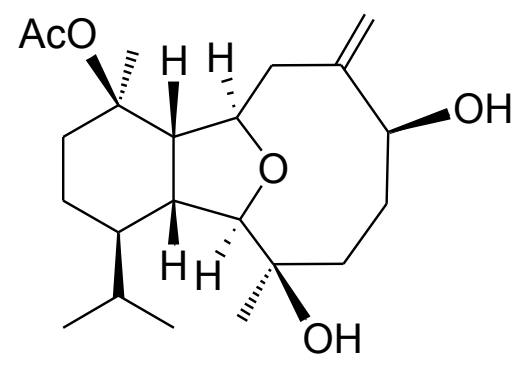

183

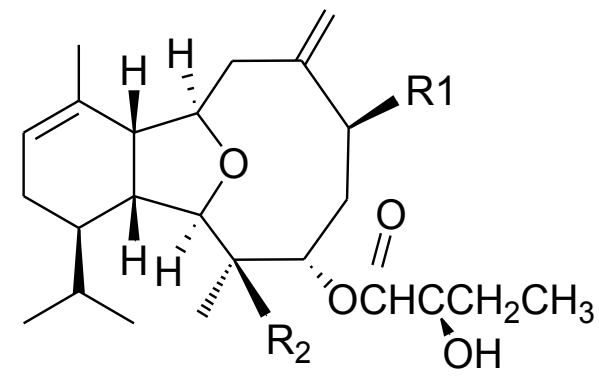

187: $\mathrm{R}_{1}=\mathrm{OH}, \mathrm{R}_{2}=\mathrm{OAc}$

188: $\mathrm{R}_{1}=\mathrm{OAc}, \mathrm{R}_{2}=\mathrm{OH}$

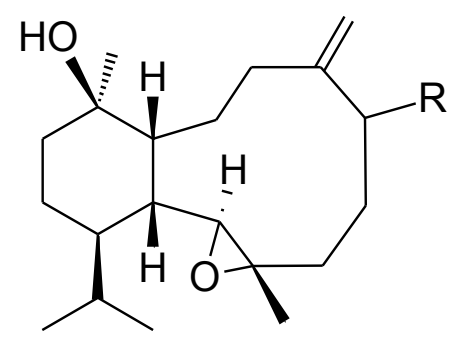

184: $\mathrm{R}=\beta-\mathrm{OH}$

185: $\mathrm{R}=\alpha-\mathrm{OH}$

\subsubsection{Briarane-based Diterpenoids}

Table 10 summarizes 35 briarane-based diterpenoids (189-223) evaluated for in vitro anti-inflammatory activity in literature published from 2008 to 2012. The corresponding chemical structures are reported in Figure 10. 
Table 10. Chemical constituents of briarane-type diterpenoids from soft corals of Taiwan.

\begin{tabular}{lcccc}
\hline No. & Name & Sources & Activities * & Reference \\
\hline $\mathbf{1 8 9}$ & Excavatolide B & Briareum excavatum & & {$[57]$} \\
$\mathbf{1 9 0}$ & Excavatolide K & Briareum excavatum & & {$[57]$} \\
$\mathbf{1 9 1}$ & Excavatolide F & Briareum excavatum & & {$[57]$} \\
$\mathbf{1 9 2}$ & Briaexcavatolide R & Briareum excavatum & & {$[57]$} \\
$\mathbf{1 9 3}$ & Excavatolide Z & Briareum excavatum & & {$[57]$} \\
$\mathbf{1 9 4}$ & Briaexcavatolide B & Briareum excavatum & & {$[57]$} \\
$\mathbf{1 9 5}$ & Briaexcavatolide K & Briareum excavatum & & {$[57]$} \\
$\mathbf{1 9 6}$ & Briaexcavatolide H & Briareum excavatum & & {$[57]$} \\
$\mathbf{1 9 7}$ & Junceol D & Junceella juncea & & {$[58]$} \\
$\mathbf{1 9 8}$ & Junceol E & Junceella juncea & $\mathrm{S}$ & {$[58]$} \\
$\mathbf{1 9 9}$ & Junceol F & Junceella juncea & $\mathrm{S}$ & {$[58]$} \\
$\mathbf{2 0 0}$ & Junceol G & Junceella juncea & $\mathrm{S}$ & {$[58]$} \\
$\mathbf{2 0 1}$ & Junceol H & Junceella juncea & $\mathrm{S}$ & {$[58]$} \\
$\mathbf{2 0 2}$ & Excavatoid L & Briareum excavatum & $\mathrm{S}, \mathrm{E}$ & {$[59]$} \\
$\mathbf{2 0 3}$ & Excavatoid M & Briareum excavatum & $\mathrm{S}, \mathrm{E}$ & {$[59]$} \\
$\mathbf{2 0 4}$ & Excavatoid N & Briareum excavatum & $\mathrm{S}, \mathrm{E}$ & {$[59]$} \\
$\mathbf{2 0 5}$ & Briarenolide F & Briareum sp. & $\mathrm{S}$ & {$[60]$} \\
$\mathbf{2 0 6}$ & Briarenolide G & Briareum sp. & & {$[60]$} \\
$\mathbf{2 0 7}$ & Fragilide J & Ellisella robusta & $\mathrm{E}$ & {$[61]$} \\
$\mathbf{2 0 8}$ & Robustolide L & Ellisella robusta & $\mathrm{S}$ & {$[61]$} \\
$\mathbf{2 0 9}$ & Briaexcavatin P & Briareum excavatum & $\mathrm{S}$ & {$[62]$} \\
$\mathbf{2 1 0}$ & Frajunolide L & Junceella fragilis & $\mathrm{S}, \mathrm{E}$ & {$[63]$} \\
$\mathbf{2 1 1}$ & Frajunolide M & Junceella fragilis & & {$[63]$} \\
$\mathbf{2 1 2}$ & Frajunolide N & Junceella fragilis & $\mathrm{E}$ & {$[63]$} \\
$\mathbf{2 1 3}$ & Frajunolide O & Junceella fragilis & $\mathrm{S}, \mathrm{E}$ & {$[63]$} \\
$\mathbf{2 1 4}$ & Juncenolide M & Junceella juncea & & {$[64]$} \\
$\mathbf{2 1 5}$ & Juncenolide N & Junceella juncea & $\mathrm{E}$ & {$[64]$} \\
$\mathbf{2 1 6}$ & Juncenolide O & Junceella juncea & $\mathrm{S}, \mathrm{E}$ & {$[64]$} \\
$\mathbf{2 1 7}$ & Frajunolide E & Junceella fragilis & $\mathrm{S}, \mathrm{E}$ & {$[65]$} \\
$\mathbf{2 1 8}$ & Frajunolide F & Junceella fragilis & & {$[65]$} \\
$\mathbf{2 1 9}$ & Frajunolide G & Junceella fragilis & & {$[65]$} \\
$\mathbf{2 2 0}$ & Frajunolide H & Junceella fragilis & & {$[65]$} \\
$\mathbf{2 2 1}$ & Frajunolide I & Junceella fragilis & & {$[65]$} \\
& Frajunolide J & Junceella fragilis & $\mathrm{S}, \mathrm{E}$ & {$[65]$} \\
$\mathbf{2 0}$ & Frajunolide K & Junceella fragilis & & {$[65]$} \\
\hline
\end{tabular}

* Inhibition of superoxide anion (S) and elastase (E). 
Figure 10. The structures of briarane-type diterpenoids (189-223).

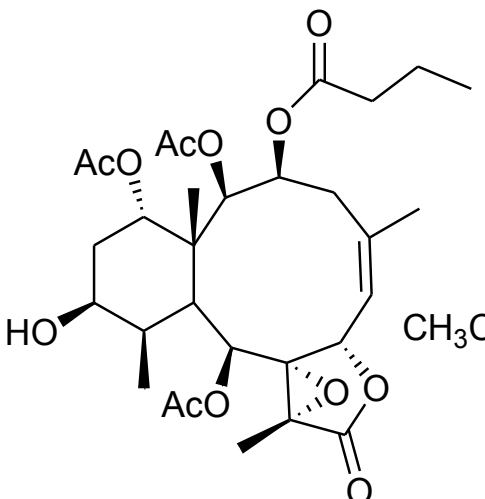

189

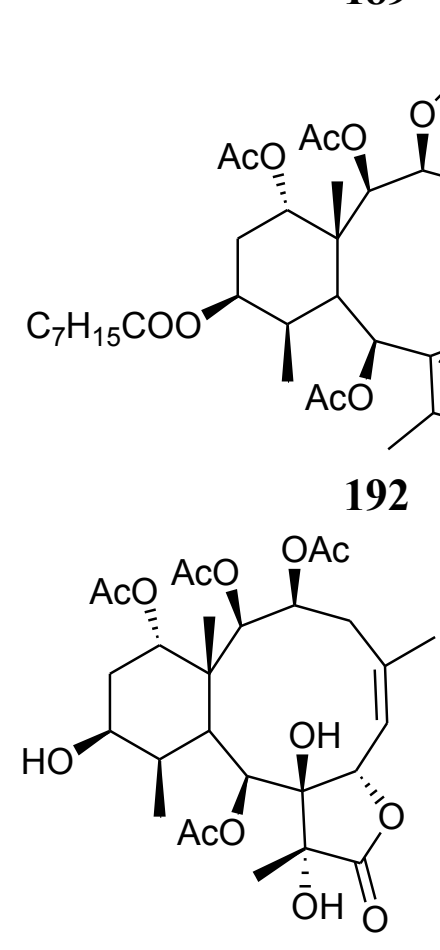

195

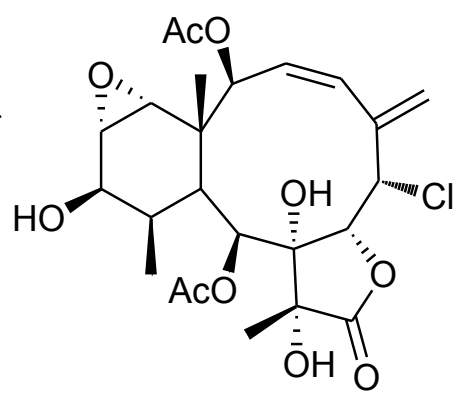

196

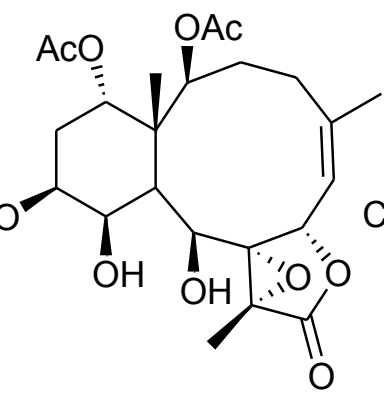

193

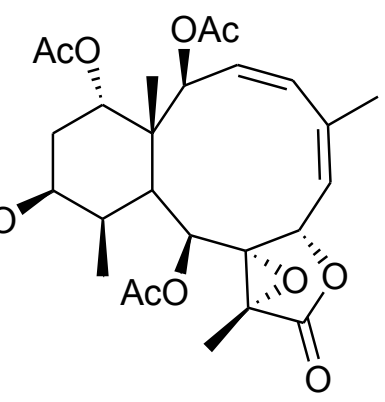

191

190

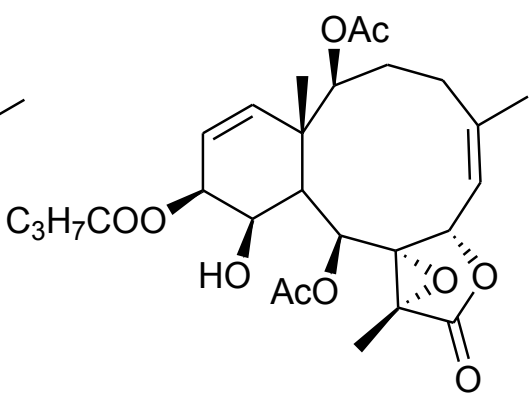

194

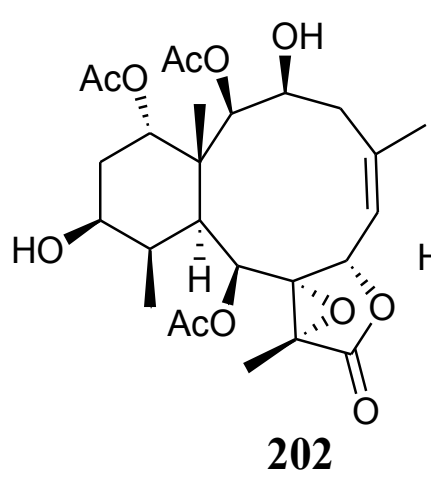

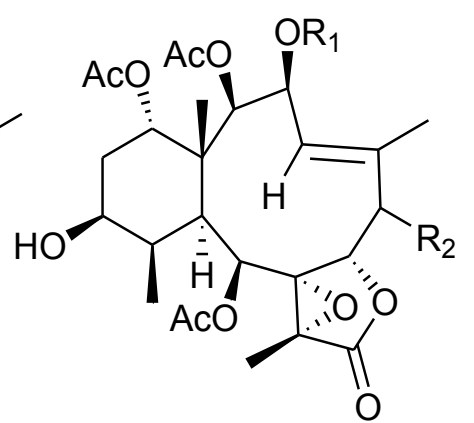

203: $\mathrm{R}_{1}=\mathrm{C}(\mathrm{O})\left(\mathrm{CH}_{2}\right)_{2} \mathrm{CH}_{3}, \mathrm{R}_{2}=\alpha-\mathrm{OH}$ 204: $\mathrm{R}_{1}=\mathrm{C}(\mathrm{O})\left(\mathrm{CH}_{2}\right)_{2} \mathrm{CH}_{3}, \mathrm{R}_{2}=\beta-\mathrm{OH}$

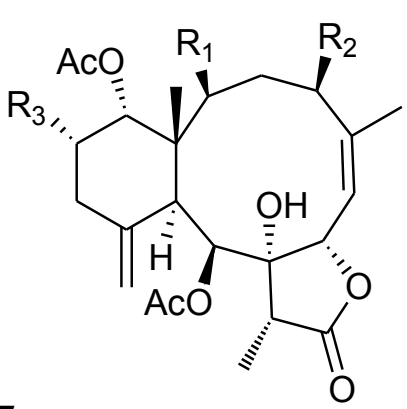

197: $\mathrm{R}_{1}=\mathrm{OC}(\mathrm{O}) \mathrm{CH}\left(\mathrm{CH}_{3}\right)_{2}$,

$$
\mathrm{R}_{2}=\mathrm{OC}(\mathrm{O}) \mathrm{CH}_{2} \mathrm{CH}\left(\mathrm{CH}_{3}\right)_{2}, \mathrm{R}_{3}=\mathrm{OAc}
$$

198: $\mathrm{R}_{1}=\mathrm{OC}(\mathrm{O}) \mathrm{CH}\left(\mathrm{CH}_{3}\right)_{2}, \mathrm{R}_{2}=\mathrm{OAc}, \mathrm{R}_{3}=\mathrm{H}$

199: $\mathrm{R}_{1}=\mathrm{OC}(\mathrm{O}) \mathrm{CH}\left(\mathrm{CH}_{3}\right) \mathrm{CH}_{2} \mathrm{CH}_{3}$,

$$
\mathrm{R}_{2}=\mathrm{OAc}, \mathrm{R}_{3}=\mathrm{H}
$$

200: $\mathrm{R}_{1}=\mathrm{OC}(\mathrm{O}) \mathrm{CH}\left(\mathrm{CH}_{3}\right) \mathrm{CH}_{2} \mathrm{CH}_{3}, \mathrm{R}_{2}=\mathrm{H}$, $\mathrm{R}_{3}=\mathrm{OAc}$

201: $\mathrm{R}=\mathrm{OAc}, \mathrm{R}_{2}=\mathrm{H}, \mathrm{R}_{3}=\mathrm{OC}(\mathrm{O}) \mathrm{CH}\left(\mathrm{CH}_{3}\right)_{2}$

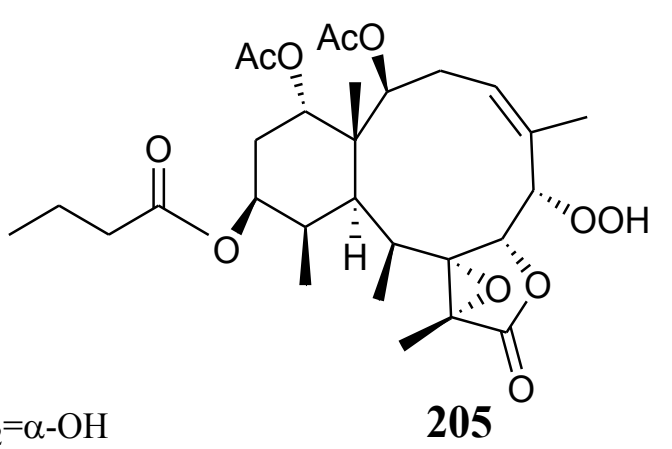


Figure 10. Cont.

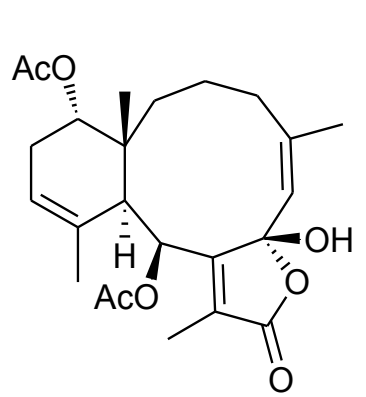

206

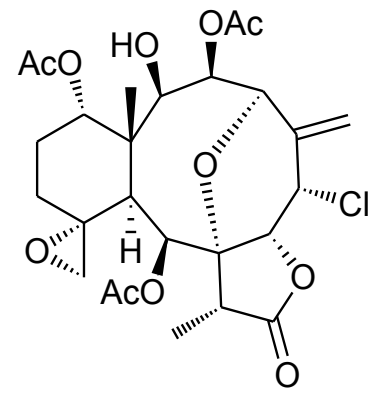

207

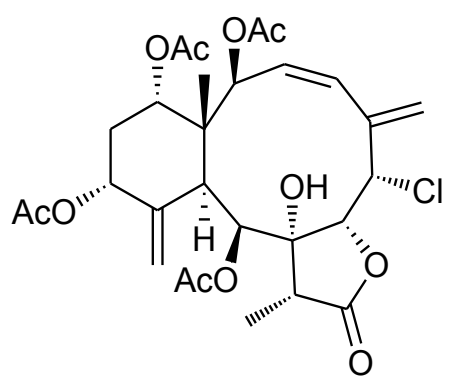

208

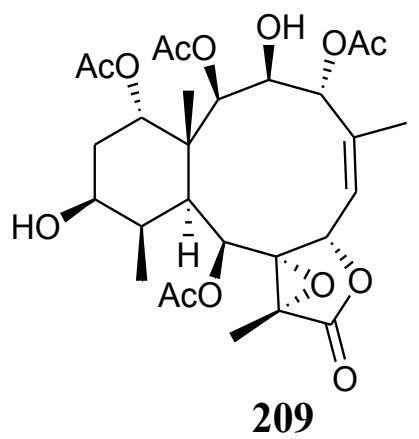

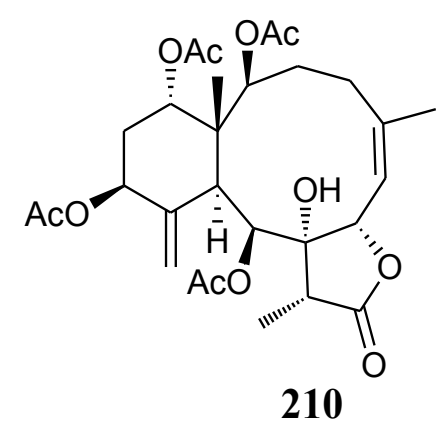

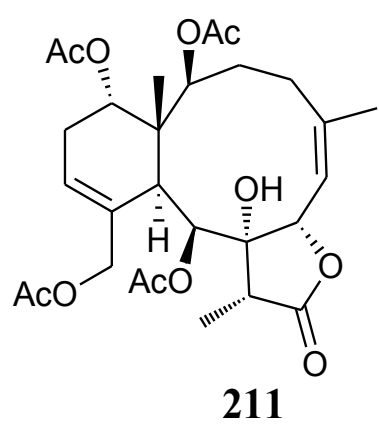

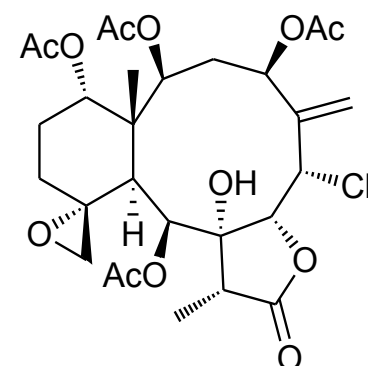

212

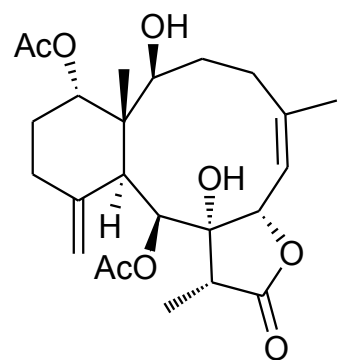

215

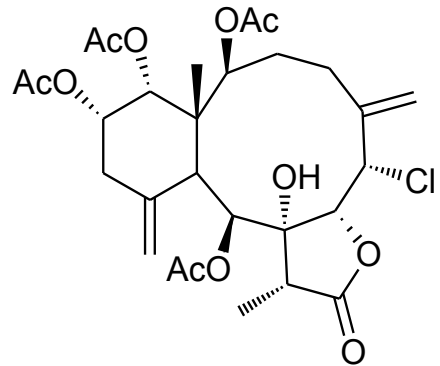

213

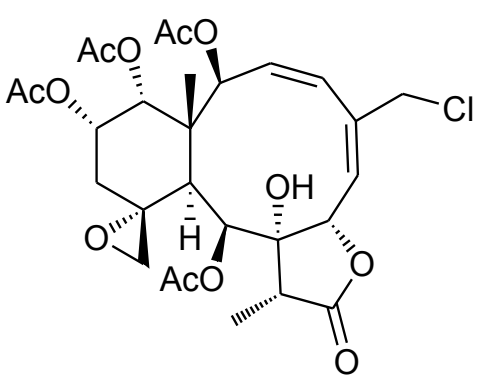

214

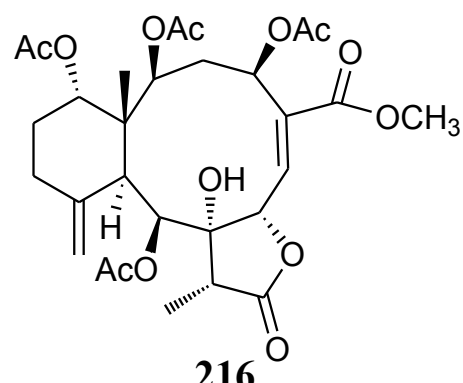

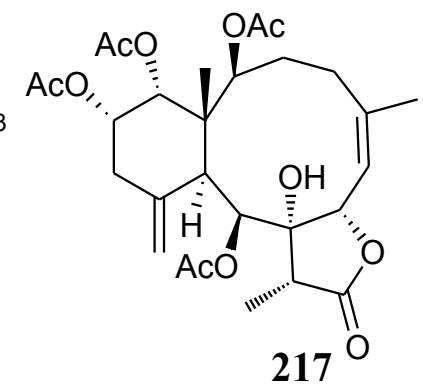

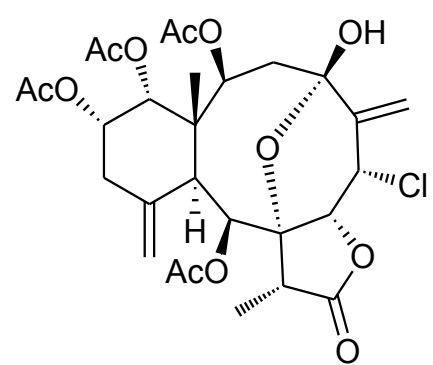

218

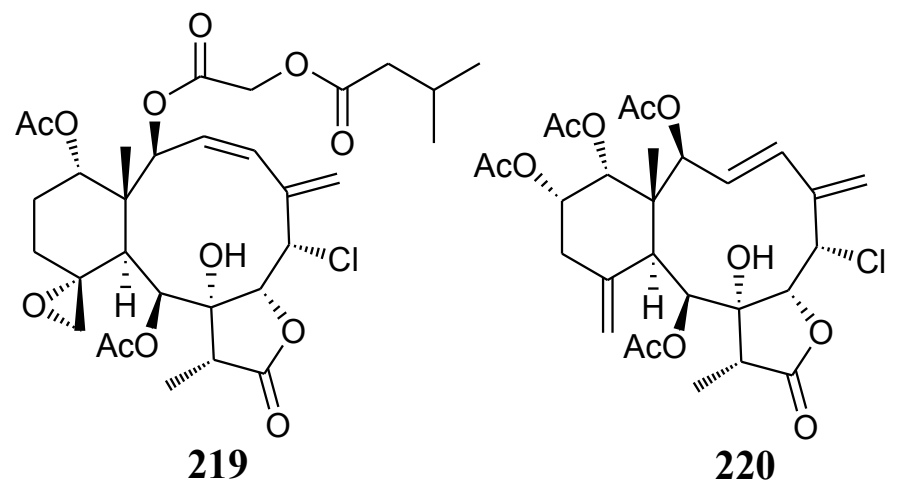


Figure 10. Cont.

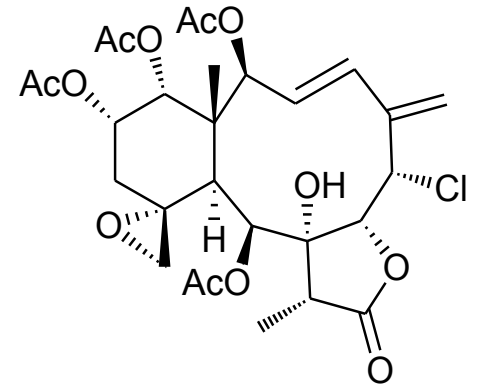

221

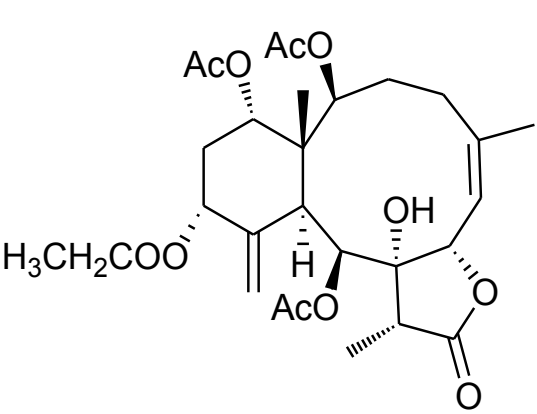

222

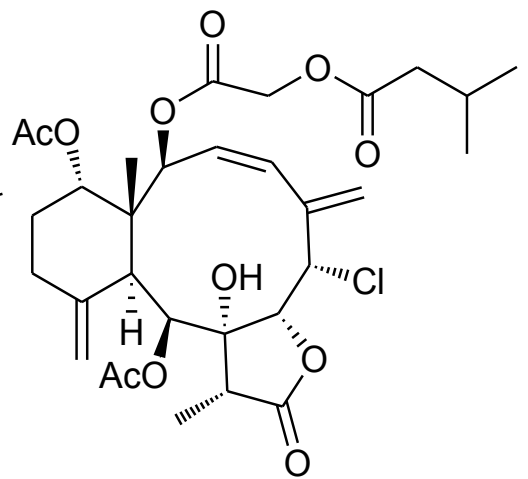

223

\subsubsection{Verticillane-Based Diterpenoids}

Table 11 summarizes 10 verticillane-based diterpenoids (224-233) evaluated for in vitro anti-inflammatory activity in literature published from 2008 to 2012. The corresponding chemical structures are reported in Figure 11.

Table 11. Chemical constituents of verticillane-type diterpenoids from soft corals of Taiwan.

\begin{tabular}{ccccc}
\hline No. & Name & Sources & Activities * & Reference \\
\hline $\mathbf{2 2 4}$ & Cespitularin R & Cespitularia hypotentaculata & & {$[66]$} \\
$\mathbf{2 2 5}$ & Cespitularin S & Cespitularia hypotentaculata & I,C & {$[66]$} \\
$\mathbf{2 2 6}$ & Cespitularin J & Cespitularia hypotentaculata & & {$[66]$} \\
$\mathbf{2 2 7}$ & Cesputularin K & Cespitularia hypotentaculata & I & {$[66]$} \\
$\mathbf{2 2 8}$ & Cespitularin M & Cespitularia hypotentaculata & & {$[66]$} \\
$\mathbf{2 2 9}$ & Cespitularin I & Cespitularia hypotentaculata & I & {$[66]$} \\
$\mathbf{2 3 0}$ & Cespitularin F & Cespitularia hypotentaculata & I & {$[66]$} \\
$\mathbf{2 3 1}$ & Cespitularin Q & Cespitularia hypotentaculata & & {$[66]$} \\
$\mathbf{2 3 2}$ & Cespitulin E & Cespitularia taenuate & S,E & {$[67]$} \\
$\mathbf{2 3 3}$ & Cespitulin G & Cespitularia taenuate & S,E & {$[67]$} \\
\hline
\end{tabular}

* Inhibition of iNOS (I), COX-2 (C), superoxide anion (S) and elastase (E).

Figure 11. The structures of verticillane-based diterpenoids (224-233).

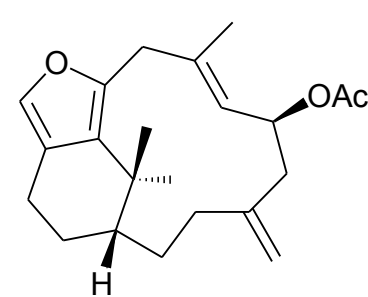

224<smiles>[R20]OC1/C=C(\C)C[C@]2([R1])OC(=O)C3=C2[C@@H](C)[C@@H](CCC(=C)C1)CC3</smiles>

225: $\mathrm{R}_{1}=\mathrm{OAc}, \mathrm{R}_{2}=\mathrm{Ac}$

226: $\mathrm{R}_{1}=\mathrm{OH}, \mathrm{R}_{2}=\mathrm{Ac}$

227: $\mathrm{R}_{1}=\mathrm{H}, \mathrm{R}_{2}=\mathrm{Ac}$

228: $\mathrm{R}_{1}=\mathrm{H}, \mathrm{R}_{2}=\mathrm{H}$

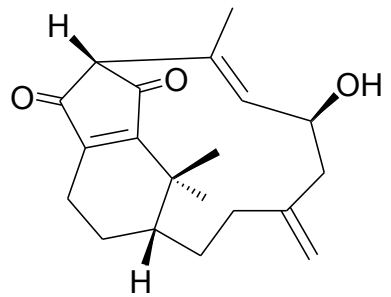

229 
Figure 11. Cont.

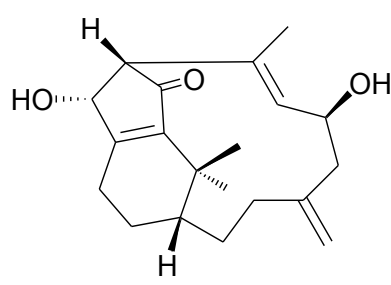

230<smiles>C=C1CC(OC(C)=O)C=C(C)CC2(O)OCC3CCC4C[C@@H]1C4C3C2(C)C</smiles>

233

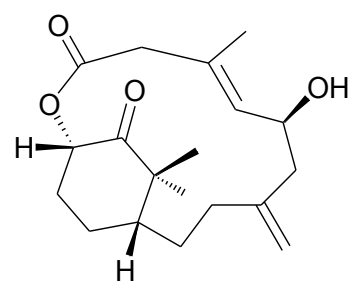

231

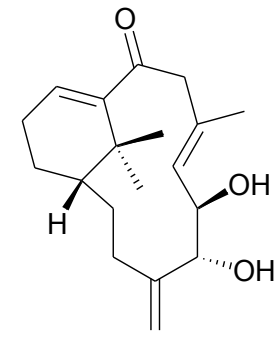

232

\subsubsection{Norditerpenoids}

Table 12 summarizes 18 norditerpenoids (234-251) evaluated for in vitro anti-inflammatory activity in literature published from 2008 to 2012. The corresponding chemical structures are reported in Figure 12.

Table 12. Chemical constituents of norditerpenoids from soft corals of Taiwan.

\begin{tabular}{|c|c|c|c|c|}
\hline No. & Name & Sources & Activities * & Reference \\
\hline 234 & Gyrosanolide A & Sinularia gyrosa & & {$[68]$} \\
\hline 235 & Gyrosanolide B & Sinularia gyrosa & I & [68] \\
\hline 236 & Gyrosanolide C & Sinularia gyrosa & I & [68] \\
\hline 237 & Gyrosanolide D & Sinularia gyrosa & & {$[68]$} \\
\hline 238 & Gyrosanolide E & Sinularia gyrosa & & [68] \\
\hline 239 & Gyrosanolide F & Sinularia gyrosa & I & [68] \\
\hline 240 & Gyrosanin A & Sinularia gyrosa & $\mathrm{I}$ & {$[68]$} \\
\hline 241 & $\begin{array}{l}\left(1 S^{*}, 5 R^{*}, 8 S^{*}, 10 R^{*}, 11 S^{*}\right)-11 \text {-Hydroxyl-1- } \\
\text { isopropenyl-8-methyl-3,6-dioxo-5,8- } \\
\text { epoxycyclotetradec-12-ene-10,12-carbonlactone } \\
\left(1 S^{*}, 5 S^{*}, 8 S^{*}, 10 R^{*}, 11 S^{*}\right)-11 \text {-Hydroxyl-1- }\end{array}$ & Sinularia gyrosa & I & {$[68]$} \\
\hline 242 & $\begin{array}{l}\text { isopropenyl-8-methyl-3,6-dioxo-5,8- } \\
\text { epoxycyclotetradec-12-ene-10,12-carbonlactone }\end{array}$ & Sinularia gyrosa & I & {$[68]$} \\
\hline 243 & Norcembrene & Sinularia gyrosa & & {$[68]$} \\
\hline 244 & epi-Norcembrene & Sinularia gyrosa & & {$[68]$} \\
\hline 245 & Leptocladolide B & Sinularia gyrosa & I & [68] \\
\hline 246 & Scabrolide D & Sinularia gyrosa & I & [68] \\
\hline 247 & Norcembrene & Sinularia gyrosa & & {$[68]$} \\
\hline 248 & Ineleganolide & Sinularia gyrosa & & [68] \\
\hline 249 & Sinulochemodin C & Sinularia gyrosa & & {$[68]$} \\
\hline 250 & Scabrolide A & Sinularia gyrosa & & [68] \\
\hline 251 & Yanarolide & Sinularia gyrosa & & [68] \\
\hline
\end{tabular}

* Inhibition of iNOS (I). 
Figure 12. The structures of norditerpenoids (234-251).
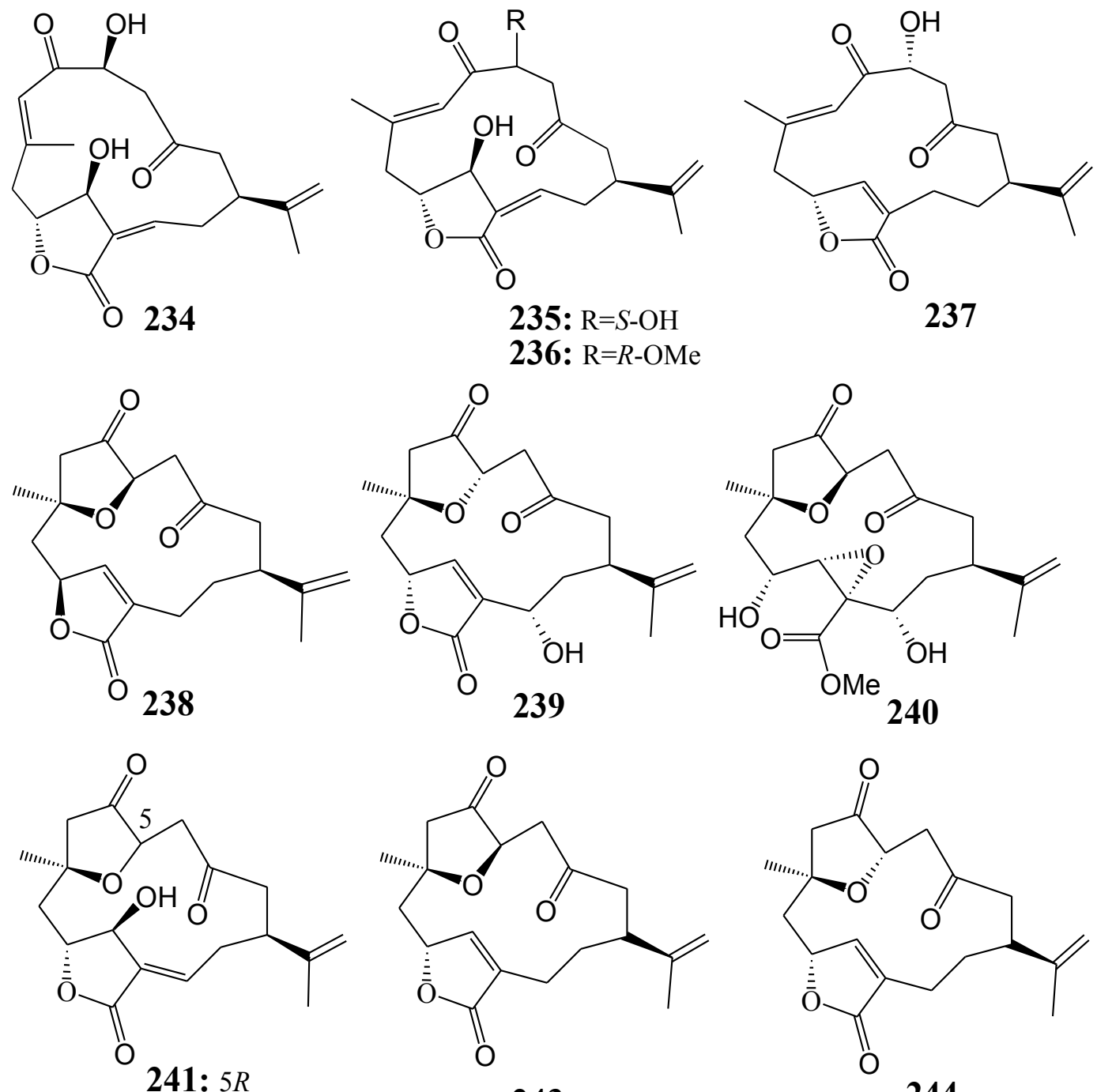

243

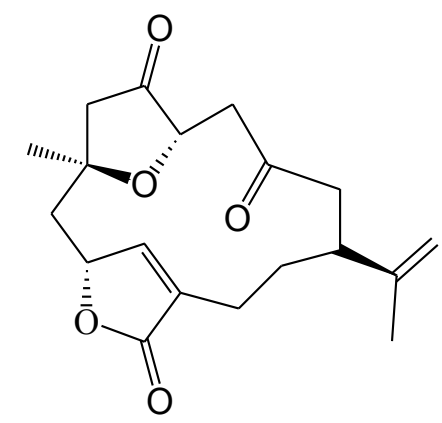

242: $5 S$
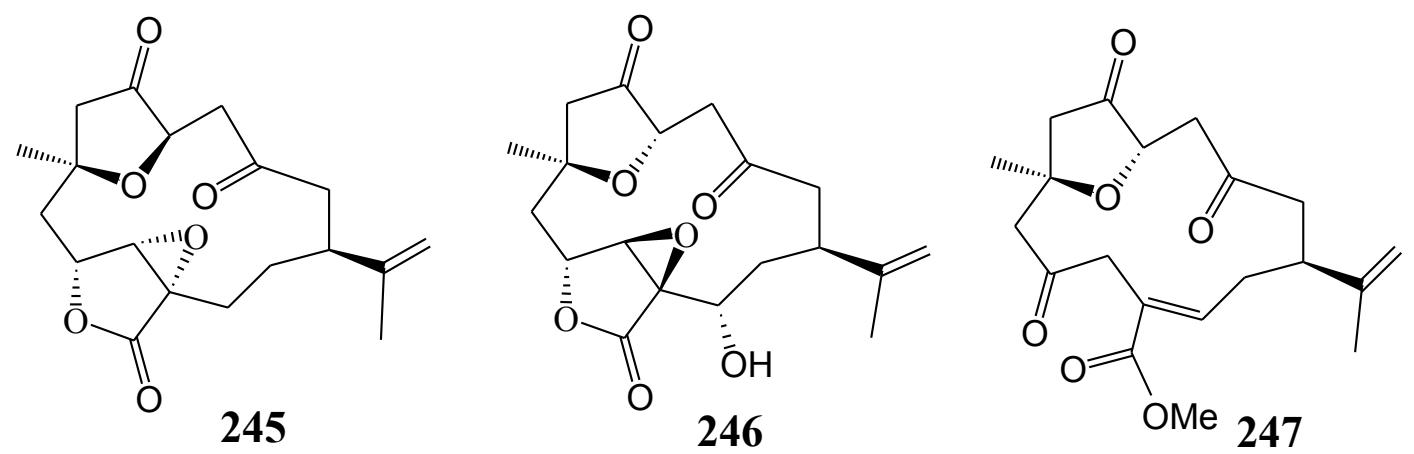

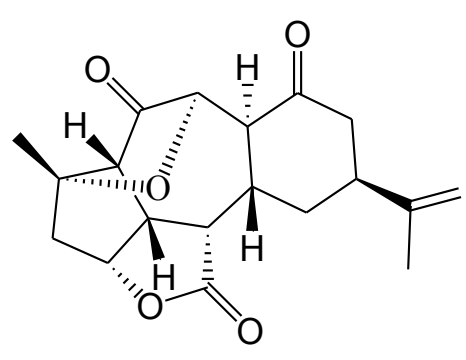

248

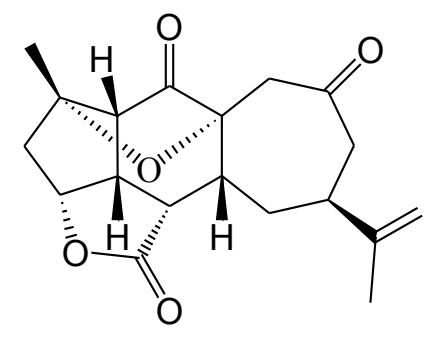

249

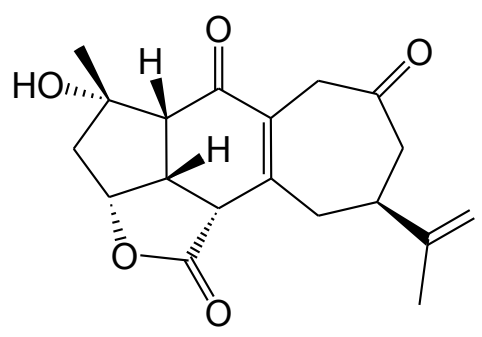

250 
Figure 12. Cont.

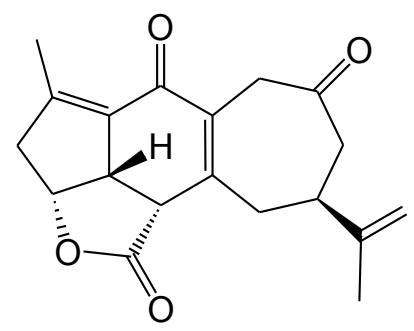

251

\subsubsection{Xenicane-Type Diterpenoids}

Table 13 summarizes six xenicane-type diterpenoids (252-257) evaluated for in vitro anti-inflammatory activity in literature published from 2008 to 2012. The corresponding chemical structures are reported in Figure 13.

Table 13. Chemical constituents of xenicane-type diterpenoids from soft corals of Taiwan.

\begin{tabular}{|c|c|c|c|c|}
\hline No. & Name & Sources & Activities * & Reference \\
\hline 252 & Asterolaurin A & Asterospicularia laurae & & [69] \\
\hline 253 & Asterolaurin B & Asterospicularia laurae & & [69] \\
\hline 254 & Asterolaurin $\mathrm{C}$ & Asterospicularia laurae & & [69] \\
\hline 255 & Asterolaurin D & Asterospicularia laurae & $\mathrm{S}, \mathrm{E}$ & [69] \\
\hline 256 & Asterolaurin E & Asterospicularia laurae & & [69] \\
\hline 257 & Asterolaurin F & Asterospicularia laurae & & [69] \\
\hline
\end{tabular}

* Inhibition of superoxide anion (S) and elastase (E).

Figure 13. The structures of xenicane-type diterpenoids (252-257).

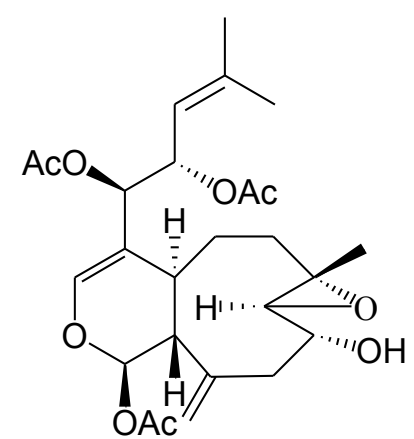

252<smiles>C=C1C[C@@H](OC(C)=O)C(C)=CC[C@@H]2/C(=C\C=C\C(C)(C)O)CO[C@H](O)[C@]12C</smiles>

255

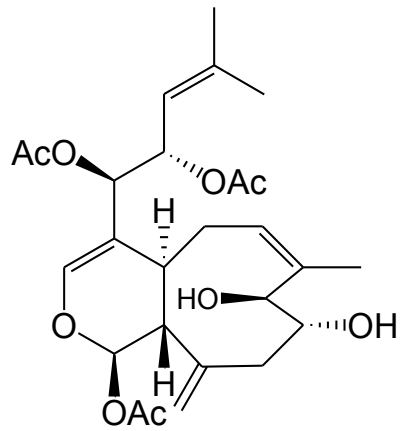

253

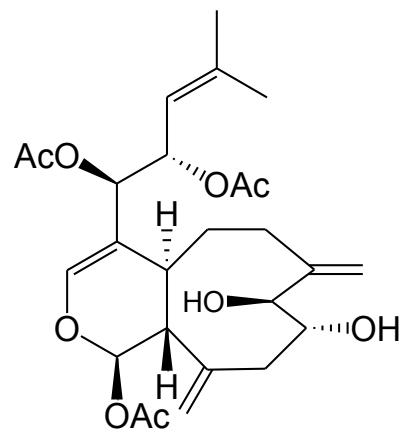

254

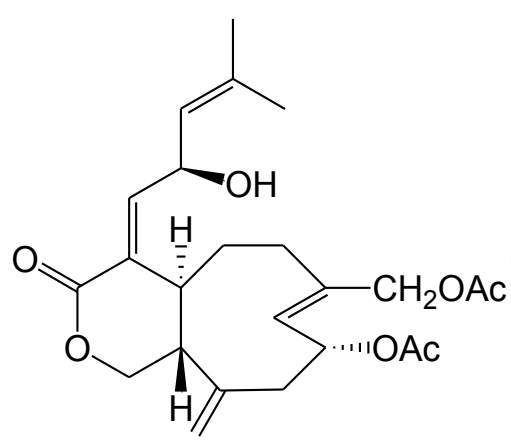

256

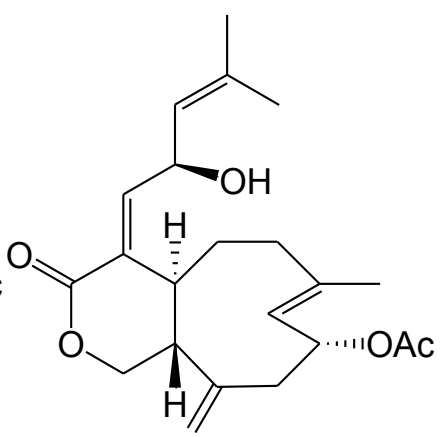

257 


\subsubsection{Other-Type Diterpenoids}

Table 14 summarizes five other-type diterpenoids (258-262) evaluated for in vitro anti-inflammatory activity in literature published from 2008 to 2012. The corresponding chemical structures are reported in Figure 14.

Table 14. Chemical constituents of other type diterpenoids from soft corals of Taiwan.

\begin{tabular}{lcccc}
\hline No. & Name & Sources & Activities * & Reference \\
\hline $\mathbf{2 5 8}$ & Gyrosanol A & Sinularia gyrosa & $\mathrm{C}$ & {$[70]$} \\
$\mathbf{2 5 9}$ & Gyrosanol B & Sinularia gyrosa & $\mathrm{C}$ & {$[70]$} \\
$\mathbf{2 6 0}$ & Echinohalimane A & Echinomuricea $\mathrm{sp}$. & $\mathrm{E}$ & {$[71]$} \\
$\mathbf{2 6 1}$ & Echinoclerodane A & Echinomuricea $\mathrm{sp}$. & $\mathrm{S}, \mathrm{E}$ & {$[72]$} \\
$\mathbf{2 6 2}$ & Echinolabdane A & Echinomuricea $\mathrm{sp}$. & & {$[73]$} \\
\hline
\end{tabular}

* Inhibition of COX-2 (C), superoxide anion (S) and elastase (E).

Figure 14. The structures of other type diterpenoids (258-262).<smiles>C=C(CCC=C(C)C)[C@H]1CC[C@H]2[C@@H](O)CC=C(C)[C@H]2C1</smiles>

258<smiles>C=C[C@]1(C)CC[C@H](C(=C)C)C[C@H]1C(=O)O</smiles><smiles>C[C@H]1CC[C@]2(C)[C@@H](C)CCC[C@]2(C)[C@@]1(C)CCC1=CC(=O)O[C@@H]1O</smiles><smiles></smiles><smiles>CC[C@]1(C)C2=CCCC(C)(C)[C@H]2CC[C@H]1C</smiles>

260

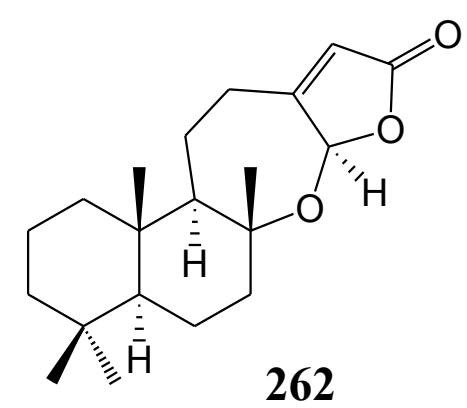

At a concentration of $10 \mu \mathrm{M}$, compounds 131, 133, 134, 139, 140-143, 147, 148, 151-153, 157-160, 162-168, 170 ceramide and cerebrosides 174, 225, 229, 230, 235, 236, 239-242, 244, 245, 258 and 259 reduced LPS-induced expression of iNOS in murine macrophage cells [46-51,66,68,70]. Compounds 134, 148, 151, 160, 163, 164, 166-168, 225, 258 and 259 suppressed the LPS-induced expression of COX-2 in these cells [46-50,66,70]. At $10 \mu \mathrm{g} / \mathrm{mL}$, compounds 180, 184, 186, 188, 198-205, 208-210, 213, 216, 217, 222, 232, 233, 255 and 261 inhibited the generation of superoxide anion by activated human neutrophils $[54-56,58-65,67,69,70,72]$. Compounds 180, 184, 186, 188, 
202-204, 207, 210, 212, 213, 215-217, 222, 232, 233, 255, 260 and 261 inhibited the release of elastase from these activated human neutrophils [53-56,59,61,63,65,67,69,71,72]. These results provided useful baseline information on the immune-regulatory and anti-oxidant activities of various marine diterpenoids. Compound 184, as 185 epimer at C-6, was showed to be more potent in the inhibition of the generation of superoxide anion and in inducing the release of elastase by active human neutrophils, suggesting that the stereochemistry at C-6 may play a key role in the above biological effects [54].

The briarane-type diterpenoid excavatolide B (189) has been demonstrated to significantly inhibit TPA-induced cutaneous inflammation activities in mice, including those related to vascular permeability, edema, and TPA-induced expression of iNOS, COX-2 and matrixmetalloproteinase-9. Excavatolide B also suppressed LPS-induced expression of TNF- $\alpha$ and IL-6 in mouse bone marrow derived dendritic cells (BMDCs) [57]. Also, excavatolide F (191), K (190) and Z (193) and briaexcavatolide B (194), H (196), K (195) and R (192) exhibited a broad spectrum of activity in inhibition of LPS-induced expression of IL-6 in BMDCs [57]. A study on the structure-activity relationship between the structures of the briarane-type diterpenoids and their inhibition of IL-6 expression in BMDCs revealed that the eight 17-epoxide of briarane-type diterpenoids may play an important role in the inhibition of IL-6 expression in specific immune cells [57]. Replacement of the C-12 hydroxyl group with long esters in briarane-type diterpenoids decreased the inhibition of IL-6 expression [57].

\subsection{Steroids}

Table 15 summarizes 60 steroids (263-322) evaluated for in vitro anti-inflammatory activity in literature published from 2008 to 2012. The corresponding chemical structures are reported in Figure 15.

Table 15. Chemical constituents of steroids from soft corals of Taiwan.

\begin{tabular}{lcccc}
\hline No. & Name & Sources & Activities * & Reference \\
\hline $\mathbf{2 6 3}$ & Stoloniferone R & Clavularia viridis & & {$[74]$} \\
$\mathbf{2 6 4}$ & Stoloniferone S & Clavularia viridis & I & {$[74]$} \\
$\mathbf{2 6 5}$ & Stoloniferone T & Clavularia viridis & I,C & {$[74]$} \\
$\mathbf{2 6 6}$ & $(25 S)$-24-Methylenecholestane- & Clavularia viridis & I,C & {$[74]$} \\
$\mathbf{2 6 7}$ & $3 \beta, 5 \alpha, 6 \beta$-triol-26-acetate & & & {$[75]$} \\
$\mathbf{2 6 8}$ & Griffinisterone A & Nephthea griffini & I & {$[75]$} \\
$\mathbf{2 6 9}$ & Griffinisterone B & Nephthea griffini & I & {$[75]$} \\
$\mathbf{2 7 0}$ & Griffinisterone C & Nephthea griffini & I & {$[75]$} \\
$\mathbf{2 7 1}$ & Griffinisterone D & Nephthea griffini & I & {$[21]$} \\
$\mathbf{2 7 2}$ & Chabrosterol & Nephthea chabroli & I,C & {$[76]$} \\
$\mathbf{2 7 3}$ & Nebrosteroid A & Nephthea chabroli & I & {$[76]$} \\
$\mathbf{2 7 4}$ & Nebrosteroid B & Nephthea chabroli & I & {$[76]$} \\
$\mathbf{2 7 5}$ & Nebrosteroid C & Nephthea chabroli & I & {$[76]$} \\
$\mathbf{2 7 6}$ & Nebrosteroid D & Nephthea chabroli & I,C & {$[76]$} \\
$\mathbf{2 7 7}$ & Nebrosteroid F & Nepthea chabroli & I,C & {$[76]$} \\
\hline
\end{tabular}


Table 15. Cont.

\begin{tabular}{|c|c|c|c|c|}
\hline 278 & Nebrosteroid G & Nepthea chabroli & $\mathrm{I}, \mathrm{C}$ & [76] \\
\hline 279 & Nebrosteroid H & Nepthea chabroli & I & [76] \\
\hline 280 & Griffinisterone F & Dendronephthya griffini & $\mathrm{I}, \mathrm{C}$ & [77] \\
\hline 281 & Griffinisterone $\mathrm{G}$ & Dendronephthya griffini & $\mathrm{I}, \mathrm{C}$ & [77] \\
\hline 282 & Griffinisterone $\mathrm{H}$ & Dendronephthya griffini & I & [77] \\
\hline 283 & Griffinipregnone & Dendronephthya griffini & $\mathrm{I}, \mathrm{C}$ & [77] \\
\hline 284 & $\begin{array}{l}1 \alpha, 3 \beta \text {-Dihydroxy- } 24 S \text { - } \\
\text { methylcholesta-5-ene }\end{array}$ & Sinularia sp. & $\mathrm{I}, \mathrm{C}$ & [78] \\
\hline 285 & $\begin{array}{c}\text { 1 } \alpha, 3 \beta \text {-Dihydroxy-24- } \\
\text { methylenecholesta-5-ene }\end{array}$ & Sinularia sp. & $\mathrm{I}, \mathrm{C}$ & [78] \\
\hline 286 & 5,24(28)-Ergostadien-3 $\beta, 23 S$-diol & Nephthea erecta & $\mathrm{I}, \mathrm{C}$ & [79] \\
\hline 287 & 5,24(28)-Ergostadien-3 $\beta, 23 R$-diol & Nephthea erecta & I & [79] \\
\hline 288 & $\begin{array}{c}(22 S)-5,24(28) \text {-Ergostadien- } \\
3 \beta, 17 \alpha, 22 \text {-triol }\end{array}$ & Nephthea erecta & $\mathrm{I}, \mathrm{C}$ & [79] \\
\hline 289 & Ergostanoid & Nephthea erecta & I & [79] \\
\hline 290 & Nebrosteroid I & Nephthea chabroli & $\mathrm{I}, \mathrm{C}$ & [80] \\
\hline 291 & Nebrosteroid J & Nephthea chabroli & $\mathrm{I}, \mathrm{C}$ & [80] \\
\hline 292 & Nebrosteroid K & Nephthea chabroli & & [80] \\
\hline 293 & Nebrosteroid L & Nephthea chabroli & $\mathrm{I}, \mathrm{C}$ & [80] \\
\hline 294 & Nebrosteroid M & Nephthea chabroli & IC & [80] \\
\hline 295 & Sarcophytosterol & Lobophytum sarcophytoides & & [38] \\
\hline 296 & $\begin{array}{c}5 \alpha, 8 \alpha \text {-Epidioxy-24- } \\
\text { methylcholesta-6-en-3 } \beta \text {-ol }\end{array}$ & Lobophytum sarcophytoides & & [38] \\
\hline 297 & $\begin{array}{c}5 \alpha, 8 \alpha \text {-Epidioxy-22,23-methylene-24- } \\
\text { methylcholest-6-en-3 } \beta \text {-ol }\end{array}$ & Lobophytum sarcophytoides & I & [38] \\
\hline 298 & Paraminabeolide A & Paraminabea acronocephala & I & [81] \\
\hline 299 & Paraminabeolide B & Paraminabea acronocephala & I & [81] \\
\hline 300 & Paraminabeolide $\mathrm{C}$ & Paraminabea acronocephala & I & [81] \\
\hline 301 & Paraminabeolide D & Paraminabea acronocephala & I & [81] \\
\hline 302 & Paraminabeolide E & Paraminabea acronocephala & & [81] \\
\hline 303 & Minabeolide-1 & Paraminabea acronocephala & $\mathrm{I}, \mathrm{C}$ & [81] \\
\hline 304 & Minabeolide-2 & Paraminabea acronocephala & $\mathrm{I}, \mathrm{C}$ & [81] \\
\hline 305 & Minabeolide-4 & Paraminabea acronocephala & $\mathrm{I}, \mathrm{C}$ & [81] \\
\hline 306 & Minabeolide-5 & Paraminabea acronocephala & $\mathrm{I}, \mathrm{C}$ & [81] \\
\hline 307 & Minabeolide-8 & Paraminabea acronocephala & & [81] \\
\hline 308 & Hirsutosterol A & Cladiella hirsuta & & [82] \\
\hline 309 & Hirsutosterol B & Cladiella hirsuta & & [82] \\
\hline 310 & Hirsutosterol C & Cladiella hirsuta & & [82] \\
\hline 311 & Hirsutosterol D & Cladiella hirsuta & & [82] \\
\hline 312 & Hirsutosterol E & Cladiella hirsuta & & [82] \\
\hline 313 & Hirsutosterol F & Cladiella hirsuta & & [82] \\
\hline 314 & Hirsutosterol G & Cladiella hirsuta & & [82] \\
\hline 315 & Crassarosterol A & Sinularia crassa & & [83] \\
\hline 316 & Crassarosteroside A & Sinularia crassa & I & [83] \\
\hline 317 & Crassarosteroside B & Sinularia crassa & I & [83] \\
\hline 318 & Crassarosteroside $\mathrm{C}$ & Sinularia crassa & I & [83] \\
\hline
\end{tabular}


Table 15. Cont.

\begin{tabular}{|c|c|c|c|c|}
\hline 319 & 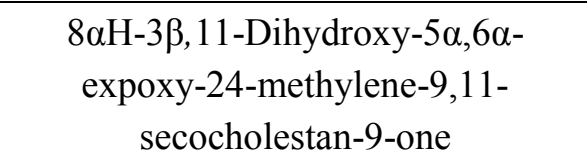 & Sinularia granosa & $\mathrm{I}, \mathrm{C}$ & [84] \\
\hline 320 & $\begin{array}{l}3 \beta, 11 \text {-Dihydroxy- } 5 \beta, 6 \beta \text {-expoxy-24- } \\
\text { methylene-9,11-secocholestan-9-one }\end{array}$ & Sinularia granosa & I & [84] \\
\hline 321 & 6-epi-Yonarasterol B & Echinomuricea sp. & $\mathrm{S}, \mathrm{E}$ & [73] \\
\hline 322 & Carijoside A & Carijoa sp. & $\mathrm{S}, \mathrm{E}$ & [85] \\
\hline
\end{tabular}

Figure 15. The structures of steroids (263-322).

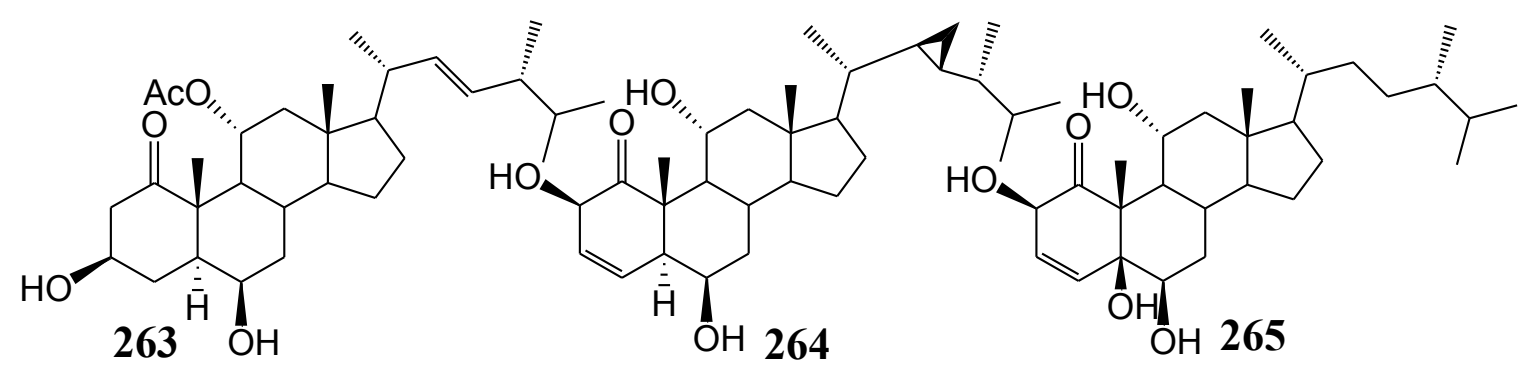

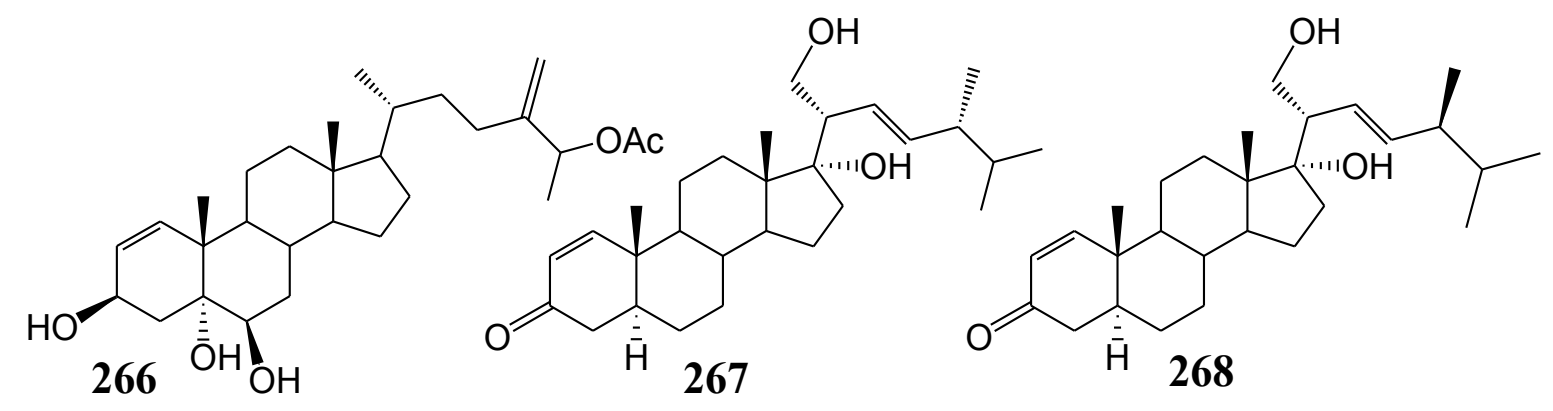

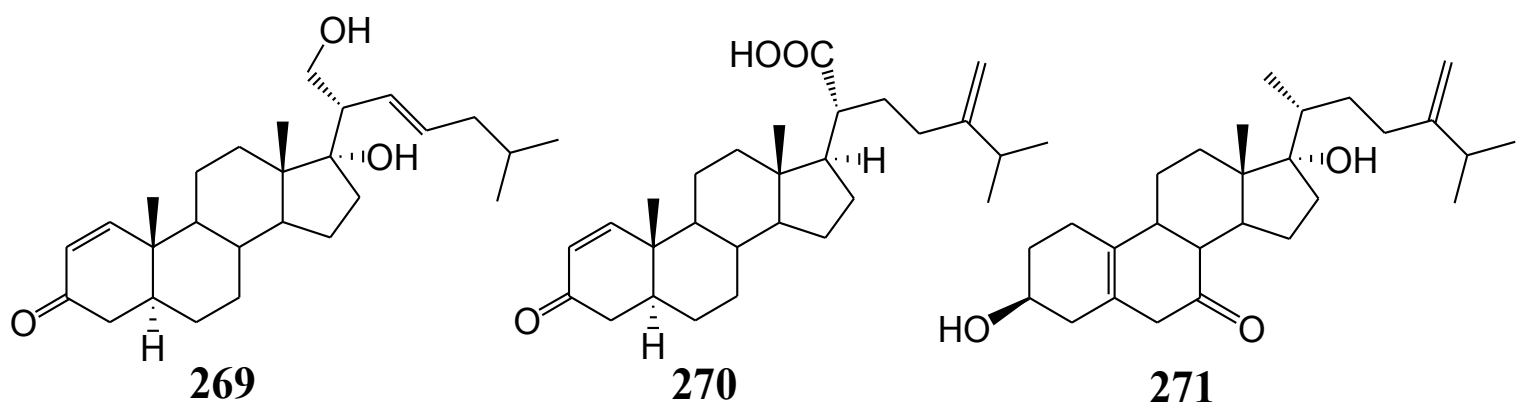

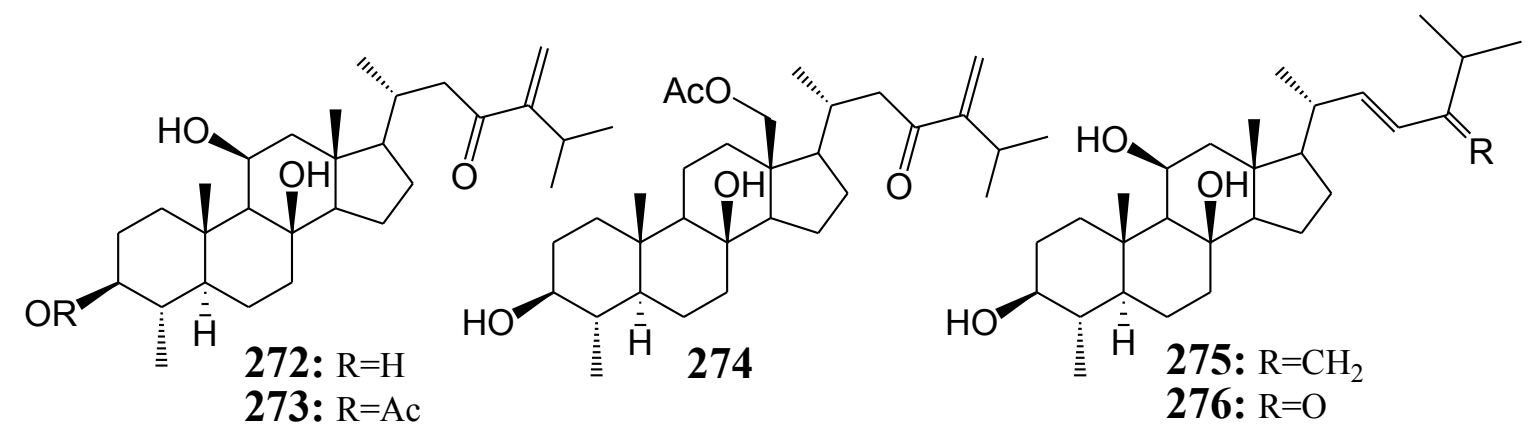


Figure 15. Cont.

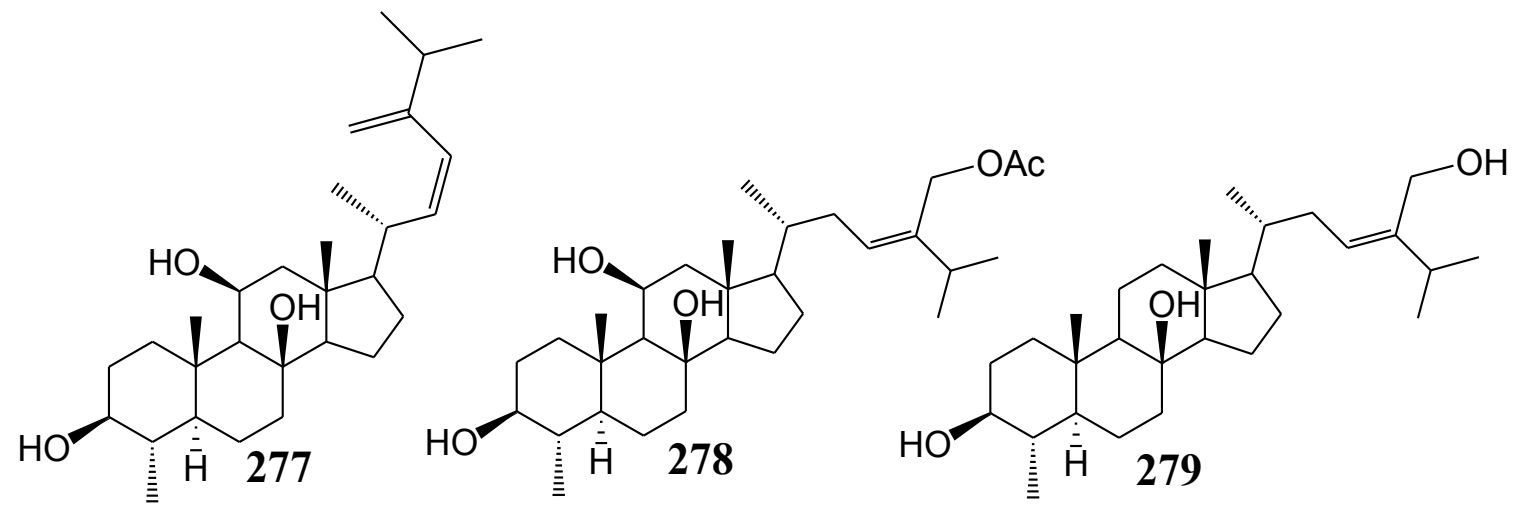

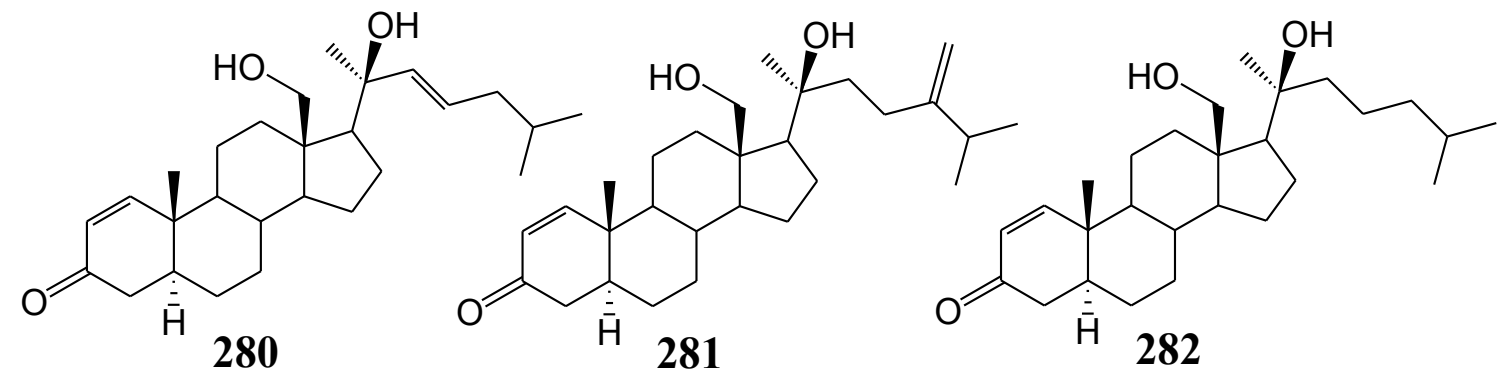<smiles>[R16]C1CCC2C3CC[C@H](C=C)[C@@]3(C)CC[C@@H]2[C@]1(C)OCCO</smiles>

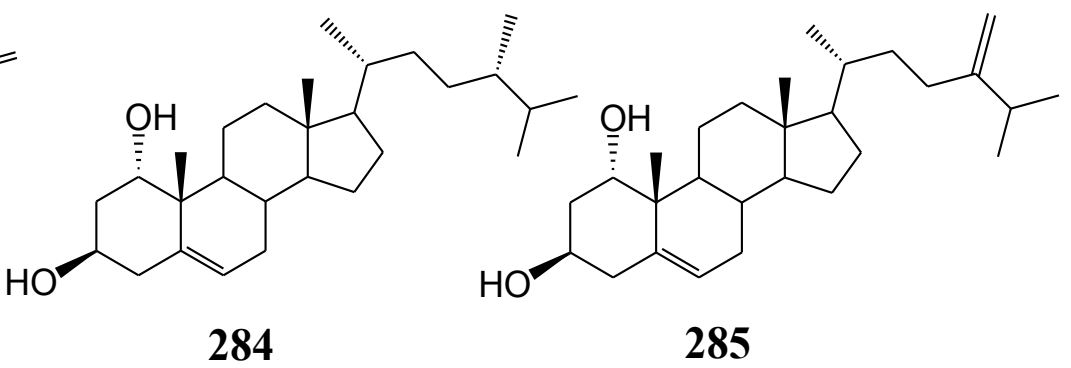

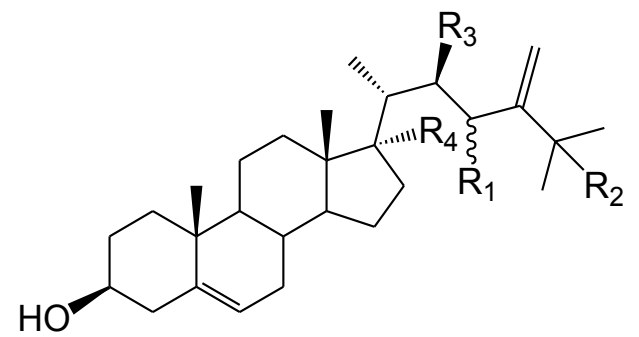

286: $\mathrm{R}_{1}=(\mathrm{S})-\mathrm{OH}, \mathrm{R}_{2}=\mathrm{H}, \mathrm{R}_{3}=\mathrm{H}, \mathrm{R}_{4}=\mathrm{H}$ 287: $\mathrm{R}_{1}=(\mathrm{R})-\mathrm{OH}, \mathrm{R}_{2}=\mathrm{H}, \mathrm{R}_{3}=\mathrm{H}, \mathrm{R}_{4}=\mathrm{H}$<smiles>C=C(CC[C@H](C)C1CCC2C3CC=C4C[C@@H](O)CC[C@]4(CO)C3CC[C@]21C(=O)OC)C(C)C</smiles>
288: $\mathrm{R}_{1}=\mathrm{H}, \mathrm{R}_{2}=\mathrm{H}, \mathrm{R}_{3}=\mathrm{OH}, \mathrm{R}_{4}=\mathrm{OH}$

289: $\mathrm{R}_{1}=\mathrm{H}, \mathrm{R}_{2}=\mathrm{OH}, \mathrm{R}_{3}=\mathrm{H}, \mathrm{R}_{4}=\mathrm{H}$

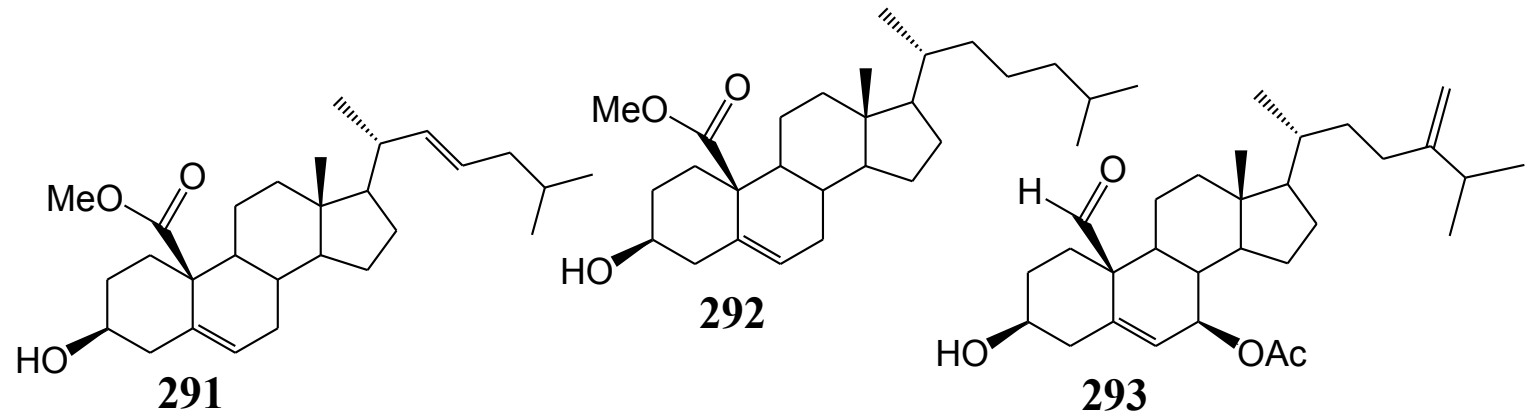


Figure 15. Cont.

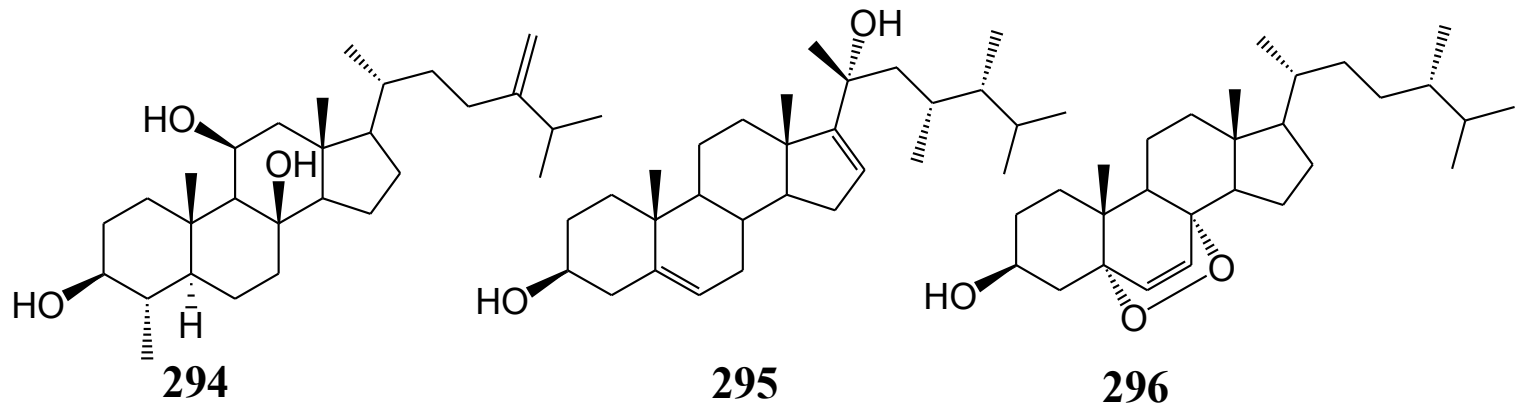<smiles>CC(=O)OC[C@]12CCC3C(CCC4=CC(=O)C=C[C@@]43C)C1CCC2[C@H](C)C[C@H]1C[C@@H](C)[C@@H](C)C(=O)O1</smiles>

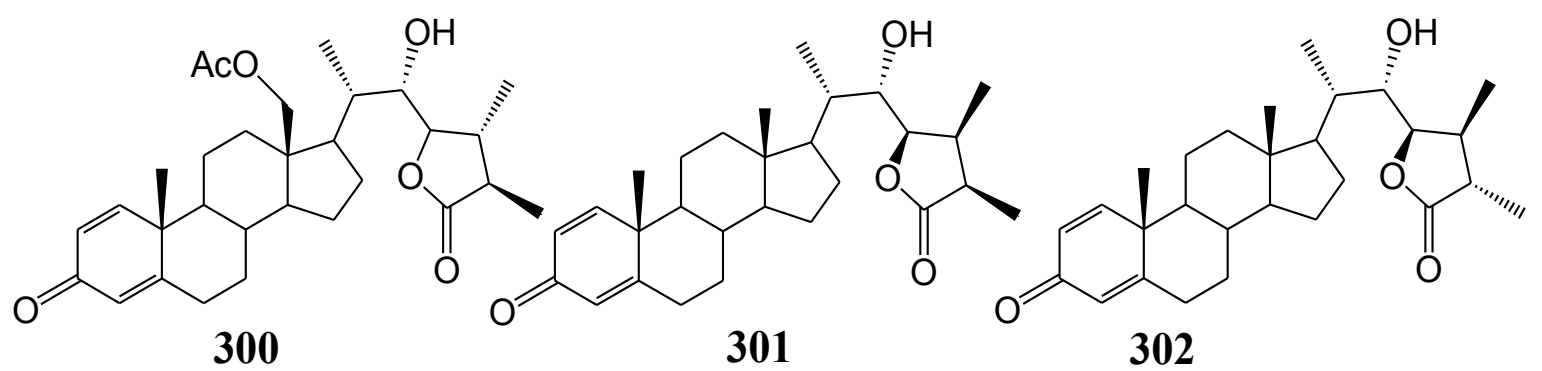

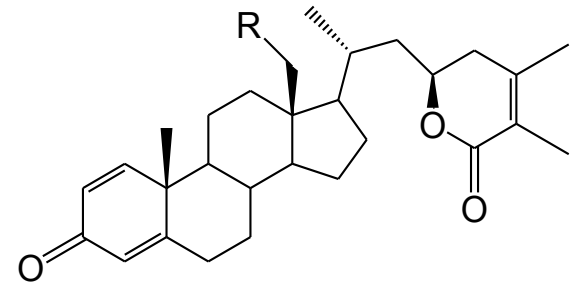

303: $\mathrm{R}=\mathrm{H}$

304: $\mathrm{R}=\mathrm{OAc}$

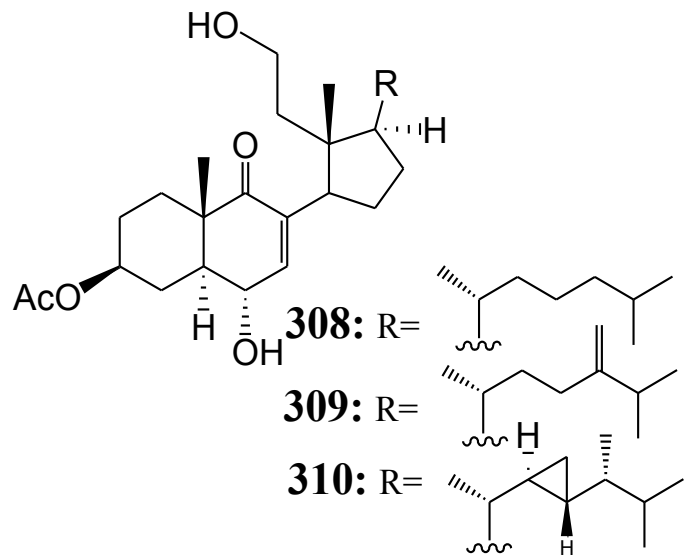

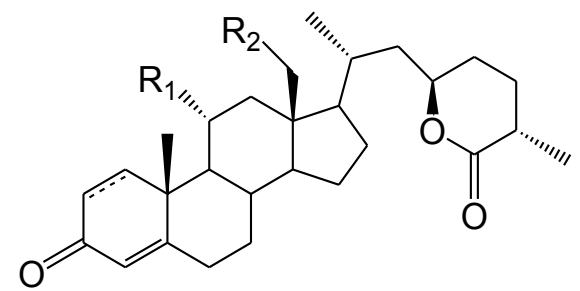

305: $\mathrm{R}_{1}=\mathrm{H}, \mathrm{R}_{2}=\mathrm{H}, \triangle^{1}$ 306: $\mathrm{R}_{1}=\mathrm{H}, \mathrm{R}_{2}=\mathrm{OAc}, \triangle^{1}$

307: $\mathrm{R}_{1}=\mathrm{OAc}, \mathrm{R}_{2}=\mathrm{H}, 1$,2-dihydro

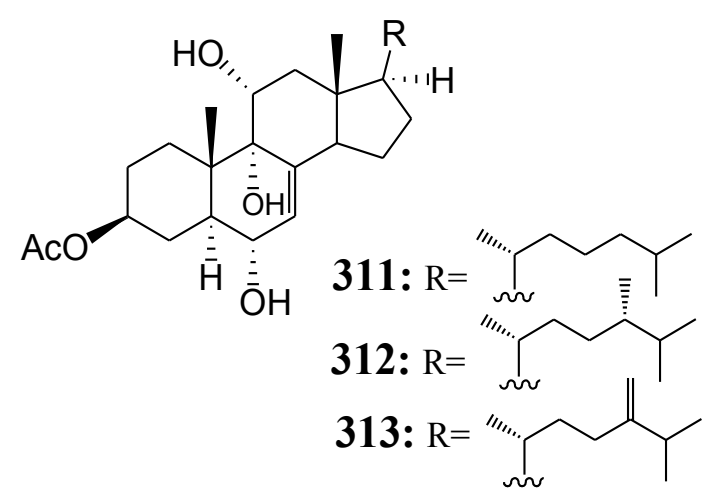


Figure 15. Cont.
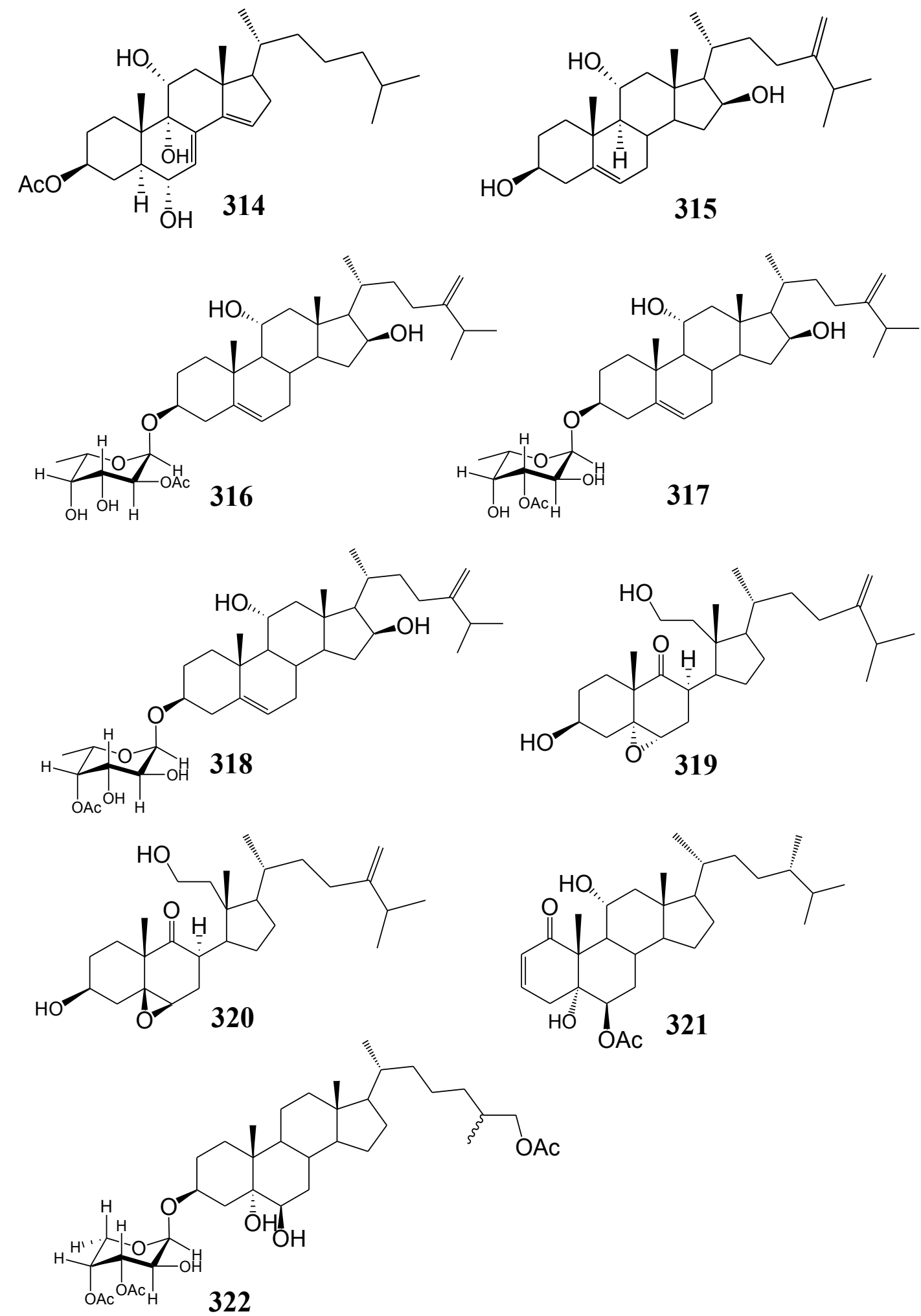

At a concentration of $10 \mu \mathrm{M}$, compounds 264-275, 277-291, 293, 294, 297, 303-307 and 316-320 reduced LPS-induced expression level of iNOS in murine macrophage cells (RAW264.7) [21,74-81,83,84]. Compounds 265, 266, 271, 275, 277, 278, 280, 281, 283-286, 288, 290, 291, 293 and 319 suppressed LPS-induced expression level of COX-2 in murine macrophage 
cells (RAW264.7) [21,74-80,84]. At $10 \mu \mathrm{g} / \mathrm{mL}$, compounds 321 and 322 inhibited the generation of superoxide anion and the release of elastase by activated human neutrophils $[73,85]$.

\subsection{Ceramide and Cerebrosides}

Table 16 summarizes ceramide (323) and five cerebrosides (324-328) evaluated for in vitro anti-inflammatory activity in literature published from 2008 to 2012. The corresponding chemical structures are reported in Figure 16.

Table 16. Chemical constituents of ceramide and cerebrosides from soft corals of Taiwan.

\begin{tabular}{lclcc}
\hline No. & Name & Sources & Activities * & Reference \\
\hline $\mathbf{3 2 3}$ & Ceramide & Sarcophyton ehrenbergi & I,C & {$[86]$} \\
$\mathbf{3 2 4}$ & Sarcoehrenoside A & Sarcophyton ehrenbergi & I & {$[86]$} \\
$\mathbf{3 2 5}$ & Sarcoehrenoside B & Sarcophyton ehrenbergi & & {$[86]$} \\
$\mathbf{3 2 6}$ & Cerebroside-3 & Sarcophyton ehrenbergi & I & {$[86]$} \\
$\mathbf{3 2 7}$ & Cerebroside-5 & Sarcophyton ehrenbergi & I & {$[86]$} \\
$\mathbf{3 2 8}$ & Cerebroside-6 & Sarcophyton ehrenbergi & I & {$[86]$} \\
\hline
\end{tabular}

* Inhibition of iNOS (I) and COX-2 (C).

Figure 16. The structures of ceramide and cerebrosides (323-328).<smiles>CCCCCCCCCCCCCCC[C@@H](O)[C@H](CO)NC(C)=O</smiles><smiles>[Z10]C[C@H](NC(=O)[C@H](O)/C=C/CCC(C)(C)C)[C@@H](O)/C=C/CC/C=C(C)/C=C/CCCCCCC</smiles>

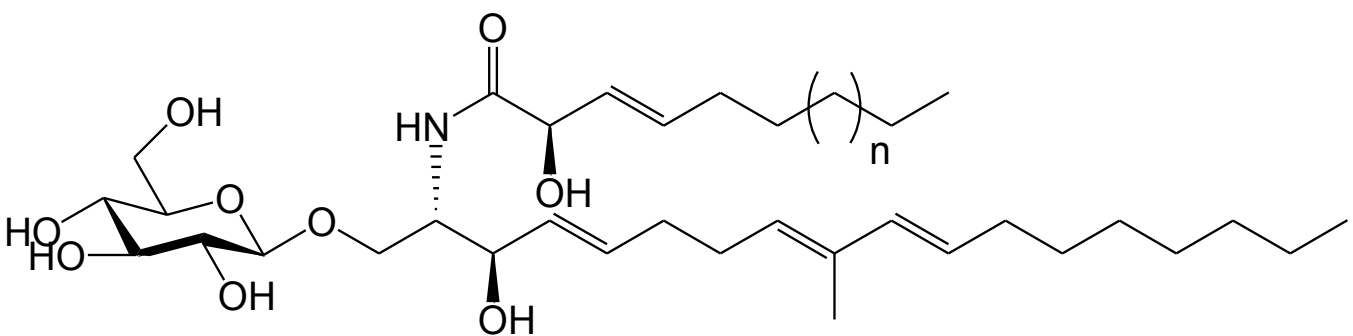

325: $n=16$

326: $n=15$

327: $n=14$

328: $n=10$ 


\subsection{Other Metabolites}

Table 17 summarizes 11 secondary metabolites of other types (329-339) evaluated for in vitro anti-inflammatory activity in literature published from 2008 to 2012. The corresponding chemical structures are reported in Figure 17.

Table 17. Chemical constituents of other metabolites from soft corals of Taiwan.

\begin{tabular}{|c|c|c|c|c|}
\hline No. & Name & Sources & Activities * & Reference \\
\hline 329 & Capilloquinone & Sinularia capillosa & I & [87] \\
\hline 330 & Capillobenzopyranol & Sinularia capillosa & I & [87] \\
\hline 331 & Capillobenzofuranol & Sinularia capillosa & & [87] \\
\hline 332 & Capillofuranocarboxylate & Sinularia capillosa & & [87] \\
\hline 333 & $\begin{array}{l}\text { (E)-5-(2,6-Dimethylocta-5,7-dienyl)furan-3- } \\
\text { carboxylic acid }\end{array}$ & Sinularia capillosa & & {$[87]$} \\
\hline 334 & $\begin{array}{l}\text { 2-[(2E,6E)-3,7-Dimethyl-8-(4-methylfuran-2-yl)octa- } \\
\text { 2,6-dienyl]-5-methylcyclohexa-2,5-diene-1,4-dione }\end{array}$ & Sinularia capillosa & $\mathrm{I}, \mathrm{C}$ & [87] \\
\hline 335 & $\begin{array}{l}\text { 2-[(2E,6E)-3,7-Dimethyl-8-(4-methylfuran-2-yl)octa- } \\
\text { 2,6-dienyl]-5-methylbenzene-1,4-diol }\end{array}$ & Sinularia capillosa & I & [87] \\
\hline 336 & (-)-Loliolide & Sinularia capillosa & & {$[87]$} \\
\hline 337 & $\begin{array}{l}\text { 3,4,11-Trimethyl-7-methylenebicyclo } \\
\text { [6.3.0] undec-2-en-11R-ol }\end{array}$ & Sinularia capillosa & & {$[87]$} \\
\hline 338 & Austrasulfone & Cladiella australis & & {$[88]$} \\
\hline 339 & Dihydroaustrasulfone alcohol & Cladiella australis & $\mathrm{I}, \mathrm{C}$ & [88] \\
\hline
\end{tabular}

* Inhibition of iNOS (I) and COX-2 (C).

Figure 17. Structures of other metabolites (329-339).<smiles>CC1=CC(=O)C(C/C=C(\C)CC/C=C(\C)CC/C=C(\C)CC[C@H](O)C(C)(C)O)=CC1=O</smiles><smiles>CO[C@](C)(CC/C=C(\C)Cc1cc(C)co1)[C@H]1Cc2cc(O)c(C)cc2O1</smiles><smiles>C=CC(=C)C(CCC(C)Cc1cc(C(=O)OC)co1)OC(C)=O</smiles> 
Figure 17. Cont.<smiles>C=C/C(C)=C/CCC(C)Cc1cc(C(=O)OC)co1</smiles>

333

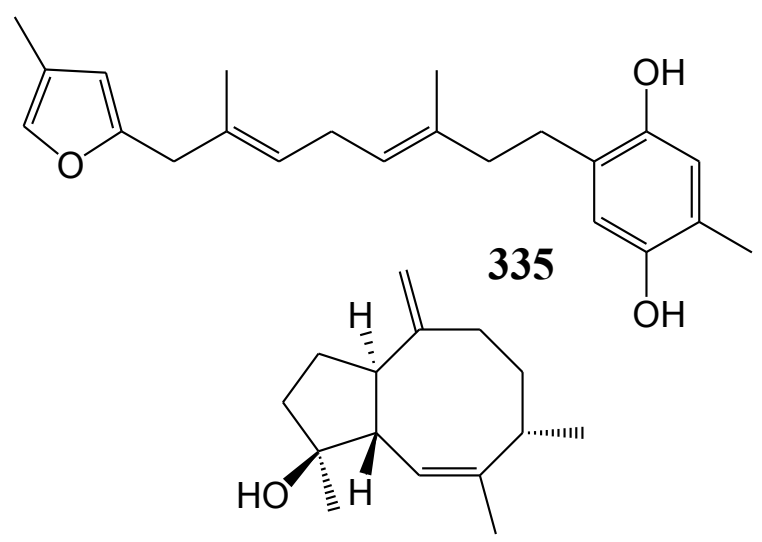<smiles>CC1=CC(=O)C(CC/C(C)=C/C/C=C(\C)Cc2cc(C)co2)=CC1=O</smiles>

337<smiles>CC(=O)CCS(=O)(=O)CCO</smiles>

339<smiles>CC1(C)C[C@H](O)C[C@]2(C)OC(=O)C=C12</smiles><smiles>C=CS(=O)(=O)CCC(C)=O</smiles>

338

At a concentration of $10 \mu \mathrm{M}$, compounds 323, 324, 326-330, 334, 335 and 339 reduced LPS-induced expression level of iNOS in murine macrophage cells (RAW264.7) [86-88]. Compounds 323, 334 and 339 suppressed LPS-induced expression levels of COX-2 in murine macrophage cells (RAW264.7) [86,88]. Austrasulfone (338) was found to exhibit a potent neuroprotective effect in human dopaminergic neuron cells (SH-SY5Y) [89,90]. In animal disease models, the synthetic precursor of austrasulfone dihydroaustrasulfone alcohol (339) was not only demonstrated to attenuate neuropathic pain, but also to suppress the progression of multiple sclerosis and atherosclerosis [88].

\section{Conclusions}

Marine invertebrates, particularly octocorals, are rich potential sources of drug leads. Most of our own and other studies on anti-inflammatory activities of natural products from soft corals have been focused on "screening-like" assays using COX-2 and iNOS as target markers. These assay studies have been useful in generating small libraries of anti-oxidant and anti-inflammatory activities from a broad spectrum of soft corals. These results, however, apparently have limitations. For example, the findings are usually generic in nature, and there is often difficulty in immediate or specific application of such results to drug/pharmaceutical discovery, as compared to the existing synthetic chemicals or phytochemicals or those being developed for clinical use. We $[45,57,88]$ and others $[25,26]$ have recently initiated a number of cross-disciplinary studies, employing bio-organic chemistry, cellular 
immunology and animal disease models for systematic and in-depth studies. As a result, we believe that useful information on the possible application of specific natural products from soft corals for future clinical studies have been obtained. We consider such approaches [57] may need to be encouraged and organized at the international level, and hopefully be integrated into systematic studies, aiming to create translational research of marine natural products for pharmaceuticals/nutraceuticals. Special emphasis may need to be placed on new or specific cell biological/disease model systems.

In terms of evaluating marine natural products for future pharmaceutical application, despite the abundance of unique marine natural products identified, the extremely low quantity of a given compound of interest that can be isolated from marine organisms may be a big hurdle for evaluation of in vivo bioactivities and development for pharmaceutical applications.

Fortunately, due to the recent advancement in aquaculture technologies, aquacultural cultivation of various types of specific soft corals is becoming possible. Our team has successfully cultured a number of species of soft corals, including Klyxum simplex and Briareum excavatum [47,91]. As a result, more abundant and routine preparations of experimental materials will become available for global distribution and collaborative research purposes. Nonetheless, the vast volume of marine organisms and the small base of knowledge so far assembled on soft coral-derived marine chemicals calls for increased international cooperation in this field.

\section{Acknowledgments}

We thank Ms. Miranda Loney of the Agricultural Biotechnology Research Center, Academia Sinica, Taiwan; and Subramanian Senthilkumar of Shanmugha Arts, Science, Technology \& Research Academy, India for editing the manuscript.

\section{Conflicts of Interest}

The authors declare no conflict of interest.

\section{References}

1. Marris, E. Marine natural products: Drugs from the deep. Nature 2006, 443, 904-905.

2. Simmons, T.L.; Andrianasolo, E.; McPhail, K.; Flatt, P.; Gerwick, W.H. Marine natural products as anticancer drugs. Mol. Cancer Ther. 2005, 4, 333-342.

3. Molinski, T.F.; Dalisay, D.S.; Lievens, S.L.; Saludes, J.P. Drug development from marine natural products. Nat. Rev. Drug Discov. 2009, 8, 69-85.

4. Hu, G.P.; Yuan, J.; Sun, L.; She, Z.G.; Wu, J.H.; Lan, X.J.; Zhu, X.; Lin, Y.C.; Chen, S.P. Statistical research on marine natural products based on data obtained between 1985 and 2008. Mar. Drugs 2011, 9, 514-525.

5. Blunt, J.W.; Copp, B.R.; Keyzers, R.A.; Munro, M.H.; Prinsep, M.R. Marine natural products. Nat. Prod. Rep. 2013, 30, 237-323.

6. Blunt, J.W.; Copp, B.R.; Keyzers, R.A.; Munro, M.H.; Prinsep, M.R. Marine natural products. Nat. Prod. Rep. 2012, 29, 144-222. 
7. Blunt, J.W.; Copp, B.R.; Munro, M.H.; Northcote, P.T.; Prinsep, M.R. Marine natural products. Nat. Prod. Rep. 2011, 28, 196-268.

8. Mayer, A.M.; Rodriguez, A.D.; Berlinck, R.G.; Fusetani, N. Marine pharmacology in 2007-8: Marine compounds with antibacterial, anticoagulant, antifungal, anti-inflammatory, antimalarial, antiprotozoal, antituberculosis, and antiviral activities affecting the immune and nervous system, and other miscellaneous mechanisms of action. Comp. Biochem. Physiol. C Toxicol. Pharmacol. 2011, 153, 191-222.

9. Kaplanski, G.; Marin, V.; Montero-Julian, F.; Mantovani, A.; Farnarier, C. IL-6: A regulator of the transition from neutrophil to monocyte recruitment during inflammation. Trends Immunol. 2003, 24, 25-29.

10. Wright, H.L.; Moots, R.J.; Bucknall, R.C.; Edwards, S.W. Neutrophil function in inflammation and inflammatory diseases. Rheumatology 2010, 49, 1618-1631.

11. Vinten-Johansen, J. Involvement of neutrophils in the pathogenesis of lethal myocardial reperfusion injury. Cardiovasc. Res. 2004, 61, 481-497.

12. Hwang, T.L.; Su, Y.C.; Chang, H.L.; Leu, Y.L.; Chung, P.J.; Kuo, L.M.; Chang, Y.J. Suppression of superoxide anion and elastase release by $\mathrm{C} 18$ unsaturated fatty acids in human neutrophils. Lipid Res. 2009, 50, 1395-1408.

13. Pham, C.T. Neutrophil serine proteases: Specific regulators of inflammation. Nat. Rev. Immunol. 2006, 6, 541-550.

14. Chang, C.H.; Wen, Z.H.; Wang, S.K.; Duh, C.Y. Capnellenes from the Formosan soft coral Capnella imbricata. J. Nat. Prod. 2008, 71, 619-621.

15. Cheng, S.Y.; Lin, E.H.; Huang, J.S.; Wen, Z.H.; Duh, C.Y. Ylangene-type and nardosinane-type sesquiterpenoids from the soft corals Lemnalia flava and Paralemnalia thyrsoides. Chem. Pharm. Bull. 2010, 58, 381-385.

16. Lu, Y.; Li, P.J.; Hung, W.Y.; Su, J.H.; Wen, Z.H.; Hsu, C.H.; Dai, C.F.; Chiang, M.Y.; Sheu, J.H. Nardosinane sesquiterpenoids from the Formosan soft coral Lemnalia flava. J. Nat. Prod. 2011, 74, 169-174.

17. Tseng, Y.J.; Shen, K.P.; Lin, H.L.; Huang, C.Y.; Dai, C.F.; Sheu, J.H. Lochmolins A-G, new sesquiterpenoids from the soft coral Sinularia lochmodes. Mar. Drugs 2012, 10, 1572-1581.

18. Huang, C.Y.; Su, J.H.; Liu, C.Y.; Wen, Z.H.; Hsu, C.H.; Chiang, M.Y.; Sheu, J.H. Oppositane-Type sesquiterpenoids from the Formosan soft coral Sinularia leptoclados. Bull. Chem. Soc. Jpn. 2010, 83, 678-682.

19. Kao, S.Y.; Su, J.H.; Hwang, T.L.; Sheu, J.H.; Wen, Z.H.; Wu, Y.C.; Sung, P.J. Menelloides C and D, new sesquiterpenoids from the Gorgonian coral Menella sp. Mar. Drugs 2011, 9, 1534-1542.

20. Lee, C.H.; Kao, C.Y.; Kao, S.Y.; Chang, C.H.; Su, J.H.; Hwang, T.L.; Kuo, Y.H.; Wen, Z.H.; Sung, P.J. Terpenoids from the octocorals Menella sp. (Plexauridae) and Lobophytum crassum (Alcyonacea). Mar. Drugs 2012, 10, 427-438.

21. Cheng, S.Y.; Huang, Y.C.; Wen, Z.H.; Chiou, S.H.; Wang, S.K.; Hsu, C.H.; Dai, C.F.; Duh, C.Y. Novel sesquiterpenes and norergosterol from the soft corals Nephthea erecta and Nephthea chabroli. Tetrahedron Lett. 2009, 50, 802-806.

22. Su, J.H.; Huang, C.Y.; Li, P.J.; Lu, Y.; Wen, Z.H.; Kao, Y.H.; Sheu, J.H. Bioactive cadinane-type compounds from the soft coral Sinularia scabra. Arch. Pharmacal. Res. 2012, 35, 779-784. 
23. Su, J.H.; Chiang, M.Y.; Wen, Z.H.; Dai, C.F.; Hsu, C.H.; Sheu, J.H. Sesquiterpenoids from the formosan soft coral Sinularia leptoclados. Chem. Pharm. Bull. 2010, 58, 250-253.

24. Kao, S.Y.; Chang, Y.C.; Su, J.H.; Lu, M.C.; Chen, Y.H.; Sheu, J.H.; Wen, Z.H.; Wang, W.H.; Kuo, Y.H.; Hwang, T.L.; et al. (-)-Hydroxylindestrenolide, a new sesquiterpenoid from a gorgonian coral Menella sp. (Plexauridae). Chem. Pharm. Bull. 2011, 59, 1048-1050.

25. Jean, Y.H.; Chen, W.F.; Duh, C.Y.; Huang, S.Y.; Hsu, C.H.; Lin, C.S.; Sung, C.S.; Chen, I.M.; Wen, Z.H. Inducible nitric oxide synthase and cyclooxygenase-2 participate in anti-inflammatory and analgesic effects of the natural marine compound lemnalol from Formosan soft coral Lemnalia cervicorni. Eur. J. Pharmacol. 2008, 578, 323-331.

26. Jean, Y.H.; Chen, W.F.; Sung, C.S.; Duh, C.Y.; Huang, S.Y.; Lin, C.S.; Tai, M.H.; Tzeng, S.F.; Wen, Z.H. Capnellene, a natural marine compound derived from soft coral, attenuates chronic constriction injury-induced neuropathic pain in rats. Br. J. Pharmacol. 2009, 158, 713-725.

27. Ahmed, A.F.; Wen, Z.H.; Su, J.H.; Hsieh, Y.T.; Wu, Y.C.; Hu, W.P.; Sheu, J.H. Oxygenated cembranoids from a Formosan soft coral Sinularia gibberosa. J. Nat. Prod. 2008, 71, 179-185.

28. Ahmed, A.F.; Tai, S.H.; Wen, Z.H.; Su, J.H.; Wu, Y.C.; Hu, W.P.; Sheu, J.H. A C-3 methylated isocembranoid and 10-oxocembranoids from a Formosan soft coral, Sinularia grandilobata. J. Nat. Prod. 2008, 71, 946-951.

29. Cheng, S.Y.; Wen, Z.H.; Chiou, S.F.; Hsu, C.H.; Wang, S.K.; Dai, C.F.; Chiang, M.Y.; Duh, C.Y. Durumolides A-E, anti-inflammatory and antibacterial cembranolides from the soft coral Lobophytum durum. Tetrahedron 2008, 64, 9698-9704.

30. Lu, Y.; Su, J.H.; Huang, C.Y.; Liu, Y.C.; Kuo, Y.H.; Wen, Z.H.; Hsu, C.H.; Sheu, J.H. Cembranoids from the soft corals Sinularia granosa and Sinularia querciformis. Chem. Pharm. Bull. 2010, 58, 464-466.

31. Chao, C.H.; Wen, Z.H.; Wu, Y.C.; Yeh, H.C.; Sheu, J.H. Cytotoxic and anti-inflammatory cembranoids from the soft coral. Lobophytum crassum. J. Nat. Prod. 2008, 71, 1819-1824.

32. Cheng, S.Y.; Wen, Z.H.; Wang, S.K.; Chiou, S.F.; Hsu, C.H.; Dai, C.F.; Chiang, M.Y.; Duh, C.Y. Unprecedented hemiketal cembranolides with anti-inflammatory activity from the soft coral. Lobophytum durum. J. Nat. Prod. 2009, 72, 152-155.

33. Cheng, S.Y.; Wen, Z.H.; Wang, S.K.; Chiou, S.F.; Hsu, C.H.; Dai, C.F.; Duh, C.Y. Anti-inflammatory cembranolides from the soft coral Lobophytum durum. Bioorg. Med. Chem. 2009, 17, 3763-3769.

34. Lin, W.Y.; Su, J.H.; Lu, Y.; Wen, Z.H.; Dai, C.F.; Kuo, Y.H.; Sheu, J.H. Cytotoxic and anti-inflammatory cembranoids from the Dongsha Atoll soft coral Sarcophyton crassocaule. Bioorg. Med. Chem. 2010, 18, 1936-1941.

35. Chen, B.W.; Chao, C.H.; Su, J.H.; Huang, C.Y.; Dai, C.F.; Wen, Z.H.; Sheu, J.H. A novel symmetric sulfur-containing biscembranoid from the Formosan soft coral Sinularia flexibilis. Tetrahedron Lett. 2010, 51, 5764-5766.

36. Su, J.H.; Wen, Z.H. Bioactive cembrane-based diterpenoids from the soft coral Sinularia triangular. Mar. Drugs 2011, 9, 944-951.

37. Lin, W.Y.; Lu, Y.; Su, J.H.; Wen, Z.H.; Dai, C.F.; Kuo, Y.H.; Sheu, J.H. Bioactive cembranoids from the dongsha atoll soft coral Sarcophyton crassocaule. Mar. Drugs 2011, 9, 994-1006. 
38. Lu, Y.; Lin, Y.C.; Wen, Z.H.; Su, J.H.; Sung, P.J.; Hsu, C.H.; Kuo, Y.H.; Chiang, M.Y.; Dai, C.F.; Sheu, J.H. Steroid and cembranoids from the Dongsha atoll soft coral Lobophytum sarcophytoides. Tetrahedron 2010, 66, 7129-7135.

39. Lu, Y.; Su, H.J.; Chen, Y.H.; Wen, Z.H.; Sheu, J.H.; Su, J.H. Anti-Inflammatory cembranoids from the Formosan soft coral Sinularia discrepans. Arch. Pharmacal. Res. 2011, 34, 1263-1267.

40. Tseng, Y.J.; Wen, Z.H.; Hsu, C.H.; Dai, C.F.; Sheu, J.H. Bioactive cembranoids from the Dongsha atoll soft coral Lobophytum crassum. Bull. Chem. Soc. Jpn. 2011, 84, 1102-1106.

41. Chao, C.H.; Chou, K.J.; Huang, C.Y.; Wen, Z.H.; Hsu, C.H.; Wu, Y.C.; Dai, C.F.; Sheu, J.H. Bioactive cembranoids from the soft coral Sinularia crassa. Mar. Drugs 2011, 9, 1955-1968.

42. Lin, W.Y.; Lu, Y.; Chen, B.W.; Huang, C.Y.; Su, J.H.; Wen, Z.H.; Dai, C.F.; Kuo, Y.H.; Sheu, J.H. Sarcocrassocolides M-O, bioactive cembranoids from the Dongsha atoll soft coral Sarcophyton crassocaule. Mar. Drugs 2012, 10, 617-626.

43. Lee, N.L.; Su, J.H. Tetrahydrofuran cembranoids from the cultured soft coral Lobophytum crassum. Mar. Drugs 2011, 9, 2526-2536.

44. Kao, C.Y.; Su, J.H.; Lu, M.C.; Hwang, T.L.; Wang, W.H.; Chen, J.J.; Sheu, J.H.; Kuo, Y.H.; Weng, C.F.; Fang, L.S.; Wen, Z.H.; Sung, P.J. Lobocrassins A-E: New cembrane-type diterpenoids from the soft coral. Lobophytum crassum. Mar. Drugs 2011, 9, 1319-1331.

45. Huang, S.Y.; Chen, N.F.; Chen, W.F.; Hung, H.C.; Lee, H.P.; Lin, Y.Y.; Wang, H.M.; Sung, P.J.; Sheu, J.H.; Wen, Z.H. Sinularin from indigenous soft coral attenuates nociceptive responses and spinal neuroinflammation in carrageenan-induced inflammatory rat model. Mar. Drugs 2012, 10, 1899-1919.

46. Wu, S.L.; Su, J.H.; Wen, Z.H.; Hsu, C.H.; Chen, B.W.; Dai, C.F.; Kuo, Y.H.; Sheu, J.H. Simplexins A-I, eunicellin-based diterpenoids from the soft coral Klyxum simplex. J. Nat. Prod. 2009, 72, 994-1000.

47. Chen, B.W.; Chao, C.H.; Su, J.H.; Tsai, C.W.; Wang, W.H.; Wen, Z.H.; Huang, C.Y.; Sung, P.J.; Wu, Y.C.; Sheu, J.H. Klysimplexins I-T, eunicellin-based diterpenoids from the cultured soft coral Klyxum simplex. Org. Biomol. Chem. 2011, 9, 834-844.

48. Chen, B.W.; Chang, S.M.; Huang, C.Y.; Chao, C.H.; Su, J.H.; Wen, Z.H.; Hsu, C.H.; Dai, C.F.; Wu, Y.C.; Sheu, J.H. Hirsutalins A-H, eunicellin-based diterpenoids from the soft coral Cladiella hirsuta. J. Nat. Prod. 2010, 73, 1785-1791.

49. Chen, B.W.; Chao, C.H.; Su, J.H.; Wen, Z.H.; Sung, P.J.; Sheu, J.H. Anti-Inflammatory eunicellin-based diterpenoids from the cultured soft coral Klyxum simplex. Org. Biomol. Chem. 2010, 8, 2363-2366.

50. Hsu, F.J.; Chen, B.W.; Wen, Z.H.; Huang, C.Y.; Dai, C.F.; Su, J.H.; Wu, Y.C.; Sheu, J.H. Klymollins A-H, bioactive eunicellin-based diterpenoids from the Formosan soft coral Klyxum molle. J. Nat. Prod. 2011, 74, 2467-2471.

51. Tai, C.J.; Su, J.H.; Huang, M.S.; Wen, Z.H.; Dai, C.F.; Sheu, J.H. Bioactive eunicellin-based diterpenoids from the soft coral Cladiella krempfi. Mar. Drugs 2011, 9, 2036-2045.

52. Chen, B.W.; Huang, C.Y.; Wen, Z.H.; Su, J.H.; Wang, W.H.; Sung, P.J.; Wu, Y.C.; Sheu, J.H. Klysimplexins U-X, eunicellin-based diterpenoids from the cultured soft coral Klyxum simplex. Bull. Chem. Soc. Jpn. 2011, 84, 1237-1242. 
53. Chen, Y.H.; Tai, C.Y.; Kuo, Y.H.; Kao, C.Y.; Li, J.J.; Hwang, T.L.; Fang, L.S.; Wang, W.H.; Sheu, J.H.; Sung, P.J. Cladieunicellins A-E, new eunicellins from an Indonesian soft coral Cladiella sp. Chem. Pharm. Bull. 2011, 59, 353-358.

54. Chen, Y.H.; Hwang, T.L.; Su, Y.D.; Chang, Y.C.; Chen, Y.H.; Hong, P.H.; Hu, L.C.; Yen, W.H.; Hsu, H.Y.; Huang, S.J.; Kuo, Y.H.; Sung, P.J. New 6-hydroxyeunicellins from a soft coral Cladiella sp. Chem. Pharm. Bull. 2012, 60, 160-163.

55. Chen, Y.H.; Tai, C.Y.; Su, Y.D.; Chang, Y.C.; Lu, M.C.; Weng, C.F.; Su, J.H.; Hwang, T.L.; Wu, Y.C.; Sung, P.J. Discovery of new eunicellins from an Indonesian octocoral Cladiella sp. Mar. Drugs 2011, 9, 934-943.

56. Chen, Y.H.; Tai, C.Y.; Hwang, T.L.; Weng, C.F.; Li, J.J.; Fang, L.S.; Wang, W.H.; Wu, Y.C.; Sung, P.J. Cladielloides A and B: New eunicellin-type diterpenoids from an Indonesian octocoral Cladiella sp. Mar. Drugs 2010, 8, 2936-2945.

57. Wei, W.C.; Lin, S.Y.; Chen, Y.J.; Wen, C.C.; Huang, C.Y.; Palanisamy, A.; Yang, N.S.; Sheu, J.H. Topical application of marine briarane-type diterpenes effectively inhibits 12-O-tetradecanoylphorbol-13-acetate-induced inflammation and dermatitis in murine skin. J. Biomed. Sci. 2011, 18, doi:10.1186/1423-0127-18-94.

58. Sung, P.J.; Pai, C.H.; Hwang, T.L.; Fan, T.Y.; Su, J.H.; Chen, J.J.; Fang, L.S.; Wang, W.H.; Sheu, J.H. Junceols D-H, new polyoxygenated briaranes from sea whip gorgonian coral Junceella juncea (Ellisellidae). Chem. Pharm. Bull. 2008, 56, 1276-1281.

59. Su, J.H.; Chen, B.Y.; Hwang, T.L.; Chen, Y.H.; Huang, I.C.; Lin, M.R.; Chen, J.J.; Fang, L.S.; Wang, W.H.; Li, J.J.; et al. Excavatoids L-N, new 12-hydroxybriaranes from the cultured octocoral Briareum excavatum (Briareidae). Chem. Pharm. Bull. 2010, 58, 662-665.

60. Hong, P.H.; Su, Y.D.; Su, J.H.; Chen, Y.H.; Hwang, T.L.; Weng, C.F.; Lee, C.H.; Wen, Z.H.; Sheu, J.H.; Lin, N.C.; et al. Briarenolides F and G, new briarane diterpenoids from a Briareum sp. octocoral. Mar. Drugs 2012, 10, 1156-1168.

61. Wang, S.H.; Chang, Y.C.; Chiang, M.Y.; Chen, Y.H.; Hwang, T.L.; Weng, C.F.; Sung, P.J. Chlorinated briarane diterpenoids from the sea whip gorgonian corals Junceella fragilis and Ellisella robusta (Ellisellidae). Chem. Pharm. Bull. 2010, 58, 928-933.

62. Sung, P.J.; Lin, M.R.; Hwang, T.L.; Fan, T.Y.; Su, W.C.; Ho, C.C.; Fang, L.S.; Wang, W.H. Briaexcavatins M-P, four new briarane-related diterpenoids from cultured octocoral Briareum excavatum (Briareidae). Chem. Pharm. Bull. 2008, 56, 930-935.

63. Liaw, C.C.; Kuo, Y.H.; Lin, Y.S.; Hwang, T.L.; Shen, Y.C. Frajunolides L-O, four new 8-hydroxybriarane diterpenoids from the gorgonian Junceella fragilis. Mar. Drugs 2011, 9, 1477-1486.

64. Chang, J.Y.; Liaw, C.C.; Fazary, A.E.; Hwang, T.L.; Shen, Y.C. New Briarane diterpenoids from the gorgonian coral Junceella juncea. Mar. Drugs 2012, 10, 1321-1330.

65. Liaw, C.C.; Shen, Y.C.; Lin, Y.S.; Hwang, T.L.; Kuo, Y.H.; Khalil, A.T. Frajunolides E-K, briarane diterpenes from Junceella fragilis. J. Nat. Prod. 2008, 71, 1551-1556.

66. Cheng, S.Y.; Lin, E.H.; Wen, Z.H.; Chiang, M.Y.; Duh, C.Y. Two new verticillane-type diterpenoids from the Formosan soft coral Cespitularia hypotentaculata. Chem. Pharm. Bull. 2010, 58, 848-851. 
67. Chang, J.Y.; Fazary, A.E.; Lin, Y.C.; Hwang, T.L.; Shen, Y.C. New verticillane diterpenoids from Cespitularia taeniata. Chem. Biodivers. 2012, 9, 654-661.

68. Cheng, S.Y.; Chuang, C.T.; Wen, Z.H.; Wang, S.K.; Chiou, S.F.; Hsu, C.H.; Dai, C.F.; Duh, C.Y. Bioactive norditerpenoids from the soft coral Sinularia gyrosa. Bioorg. Med. Chem. 2010, 18, 3379-3386.

69. Lin, Y.C.; Abd El-Razek, M.H.; Hwang, T.L.; Chiang, M.Y.; Kuo, Y.H.; Dai, C.F.; Shen, Y.C. Asterolaurins A-F, xenicane diterpenoids from the Taiwanese soft coral Asterospicularia laurae. J. Nat. Prod. 2009, 72, 1911-1916.

70. Cheng, S.Y.; Chuang, C.T.; Wang, S.K.; Wen, Z.H.; Chiou, S.F.; Hsu, C.H.; Dai, C.F.; Duh, C.Y. Antiviral and anti-inflammatory diterpenoids from the soft coral Sinularia gyrosa. J. Nat. Prod. 2010, 73, 1184-1187.

71. Chung, H.M.; Hu, L.C.; Yen, W.H.; Su, J.H.; Lu, M.C.; Hwang, T.L.; Wang, W.H.; Sung, P.J. Echinohalimane A, a bioactive halimane-type diterpenoid from a Formosan gorgonian Echinomuricea sp. (Plexauridae). Mar. Drugs 2012, 10, 2246-2253.

72. Cheng, C.H.; Chung, H.M.; Hwang, T.L.; Lu, M.C.; Wen, Z.H.; Kuo, Y.H.; Wang, W.H.; Sung, P.J. Echinoclerodane A: A new bioactive clerodane-type diterpenoid from a gorgonian coral Echinomuricea sp. Molecules 2012, 17, 9443-9450.

73. Chung, H.M.; Hong, P.H.; Su, J.H.; Hwang, T.L.; Lu, M.C.; Fang, L.S.; Wu, Y.C.; Li, J.J.; Chen, J.J.; Wang, W.H.; et al. Bioactive compounds from a gorgonian coral Echinomuricea sp. (Plexauridae). Mar. Drugs 2012, 10, 1169-1179.

74. Chang, C.H.; Wen, Z.H.; Wang, S.K.; Duh, C.Y. New anti-inflammatory steroids from the Formosan soft coral Clavularia viridis. Steroids 2008, 73, 562-567.

75. Chao, C.H.; Wen, Z.H.; Chen, I.M.; Su, J.H.; Huang, H.C.; Chiang, M.Y.; Sheu, J.H. Anti-inflammatory steroids from the octocoral Dendronephthya griffini. Tetrahedron 2008, 64, 3554-3560.

76. Huang, Y.C.; Wen, Z.H.; Wang, S.K.; Hsu, C.H.; Duh, C.Y. New anti-inflammatory 4-methylated steroids from the Formosan soft coral Nephthea chabroli. Steroids 2008, 73, 1181-1186.

77. Chao, C.H.; Wen, Z.H.; Su, J.H.; Chen, I.M.; Huang, H.C.; Dai, C.F.; Sheu, J.H. Further study on anti-inflammatory oxygenated steroids from the octocoral Dendronephthya griffini. Steroids 2008, $73,1353-1358$.

78. Su, J.H.; Lo, C.L.; Lu, Y.; Wen, Z.H.; Huang, C.Y.; Dai, C.F.; Sheu, J.H. Anti-Inflammatory polyoxygenated steroids from the soft coral Sinularia sp. Bull. Chem. Soc. Jpn. 2008, 81, 1616-1620.

79. Cheng, S.Y.; Wen, Z.H.; Wang, S.K.; Chiang, M.Y.; El-Gamal, A.A.; Dai, C.F.; Duh, C.Y. Revision of the absolute configuration at $\mathrm{C}(23)$ of lanostanoids and isolation of secondary metabolites from Formosan soft coral Nephthea erecta. Chem. Biodivers. 2009, 6, 86-95.

80. Cheng, S.Y.; Huang, Y.C.; Wen, Z.H.; Hsu, C.H.; Wang, S.K.; Dai, C.F.; Duh, C.Y. New 19-oxygenated and 4-methylated steroids from the Formosan soft coral Nephthea chabroli. Steroids 2009, 74, 543-547. 
81. Chao, C.H.; Chou, K.J.; Wen, Z.H.; Wang, G.H.; Wu, Y.C.; Dai, C.F.; Sheu, J.H. Paraminabeolides A-F, cytotoxic and anti-inflammatory marine withanolides from the soft coral Paraminabea acronocephala. J. Nat. Prod. 2011, 74, 1132-1141.

82. Chen, B.W.; Chang, S.M.; Huang, C.Y.; Su, J.H.; Wen, Z.H.; Wu, Y.C.; Sheu, J.H. Hirsutosterols A-G, polyoxygenated steroids from a Formosan soft coral Cladiella hirsuta. Org. Biomol. Chem. 2011, 9, 3272-3278.

83. Chao, C.H.; Chou, K.J.; Huang, C.Y.; Wen, Z.H.; Hsu, C.H.; Wu, Y.C.; Dai, C.F.; Sheu, J.H. Steroids from the soft coral Sinularia crassa. Mar. Drugs 2012, 10, 439-450.

84. Huang, C.Y.; Su, J.H.; Duh, C.Y.; Chen, B.W.; Wen, Z.H.; Kuo, Y.H.; Sheu, J.H. A new 9,11-secosterol from the soft coral Sinularia granosa. Bioorg. Med. Chem. Lett. 2012, 22, 4373-4376.

85. Liu, C.Y.; Hwang, T.L.; Lin, M.R.; Chen, Y.H.; Chang, Y.C.; Fang, L.S.; Wang, W.H.; Wu, Y.C.; Sung, P.J. Carijoside A, a bioactive sterol glycoside from an octocoral Carijoa sp. (Clavulariidae). Mar. Drugs 2010, 8, 2014-2020.

86. Cheng, S.Y.; Wen, Z.H.; Chiou, S.F.; Tsai, C.W.; Wang, S.K.; Hsu, C.H.; Dai, C.F.; Chiang, M.Y.; Wang, W.H.; Duh, C.Y. Ceramide and cerebrosides from the octocoral Sarcophyton ehrenbergi. J. Nat. Prod. 2009, 72, 465-468.

87. Cheng, S.Y.; Huang, K.J.; Wang, S.K.; Wen, Z.H.; Chen, P.W.; Duh, C.Y. Antiviral and anti-inflammatory metabolites from the soft coral Sinularia capillosa. J. Nat. Prod. 2010, 73, $771-775$.

88. Wen, Z.H.; Chao, C.H.; Wu, M.H.; Sheu, J.H. A neuroprotective sulfone of marine origin and the in vivo anti-inflammatory activity of an analogue. Eur. J. Med. Chem. 2010, 45, 5998-6004.

89. Kitamura, Y.; Kosaka, T.; Kakimura, J.I.; Matsuoka,Y.; Kohno, Y.; Nomura,Y.; Taniguchi, T. Protective effects of the antiparkinsonian drugs talipexole and pramipexole against 1-methyl-4-phenylpyridinium-induced apoptotic death in human neuroblastoma SH-SY5Y cells. Mol. Pharmacol. 1998, 54, 1046-1054.

90. Blum, D.; Torch, S.; Lambeng, N.; Nissou, M.; Benabid, A.L.; Sadoul, R.; Verna, J.M. Molecular pathways involved in the neurotoxicity of 6-OHDA, dopamine and MPTP: Contribution to the apoptotic theory in Parkinson's disease. Prog. Neurobiol. 2001, 65, 137-172.

91. Sung, P.J.; Chen, B.Y.; Lin, M.R.; Hwang, T.L.; Wang, W.H.; Sheu, J.H.; Wu, Y.C. Excavatoids E and F: Discovery of two new briaranes from the cultured octocoral Briareum excavatum. Mar. Drugs 2009, 7, 472-482.

(C) 2013 by the authors; licensee MDPI, Basel, Switzerland. This article is an open access article distributed under the terms and conditions of the Creative Commons Attribution license (http://creativecommons.org/licenses/by/3.0/). 